\title{
Nuclear imaging for improved radioembolization treatment planning
}

Nucleaire beeldvorming voor verbeterde behandelplanning van radioembolisatie 


\section{Nuclear imaging for improved radioembolization treatment planning}

Nucleaire beeldvorming voor verbeterde behandelplanning van radioembolisatie

(met een samenvatting in het Nederlands)

Nuclear imaging for improved radioembolization treatment planning

PhD thesis, Utrecht University

(c) B. Kunnen, Utrecht, 2020

All rights reserved. No part of this publication may be reproduced, stored in a retrieval system, or transmitted, in any form or by any means, without permission in writing from the author. The copyright of the articles that have been published or have been accepted for publication has been transferred to the respective journals.

ISBN: 978-94-93184-73-2

Cover design and lay-out: Guus Gijben | Proefschrift-aio.nl

Printed by: Proefschrift-aio.nl

\author{
Proefschrift \\ ter verkrijging van de graad van doctor aan de

$$
\text { Universiteit Utrecht }
$$$$
\text { op gezag van de }
$$ \\ rector magnificus, prof.dr. H.R.B.M. Kummeling, \\ ingevolge het besluit van het college voor promoties \\ in het openbaar te verdedigen op \\ dinsdag 24 november 2020 des ochtends te 11.00 uur
}

Britt Kunnen

geboren op 16 september 1990

te Amsterdam 


\section{Promotoren:}

Prof. dr. ir. H.W.A.M. de Jong

Prof. dr. M.G.E.H. Lam

Prof. dr. ir. M.A. Viergever

\section{Content}

Chapter 1 - Introduction

Chapter $\mathbf{2}$ - Technical advances in image guidance of radionuclide therapy

Chapter 3 - A dual layer detector for simultaneous fluoroscopic and nuclear imaging 37

Chapter 4 - Comparison of three different therapeutic particles in radioembolization of locally advanced intrahepatic cholangiocarcinoma

Chapter 5 - Radioembolization lung shunt estimation based on a ${ }^{90} Y$ pre-treatment procedure: a phantom study

Chapter 6 - Feasibility of imaging ${ }^{90} Y$ microspheres at diagnostic activity levels for hepatic radioembolization treatment planning

Chapter 7 - Comparison of the Biograph Vision and Biograph mCT for quantitative ${ }^{90}$ Y PET/CT imaging for radioembolization

Chapter 8 - General discussion

Chapter 9 - Summary en Nederlandse samenvatting

References

Appendix

List of publications

CV

Dankwoord

49

150

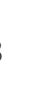


Introduction
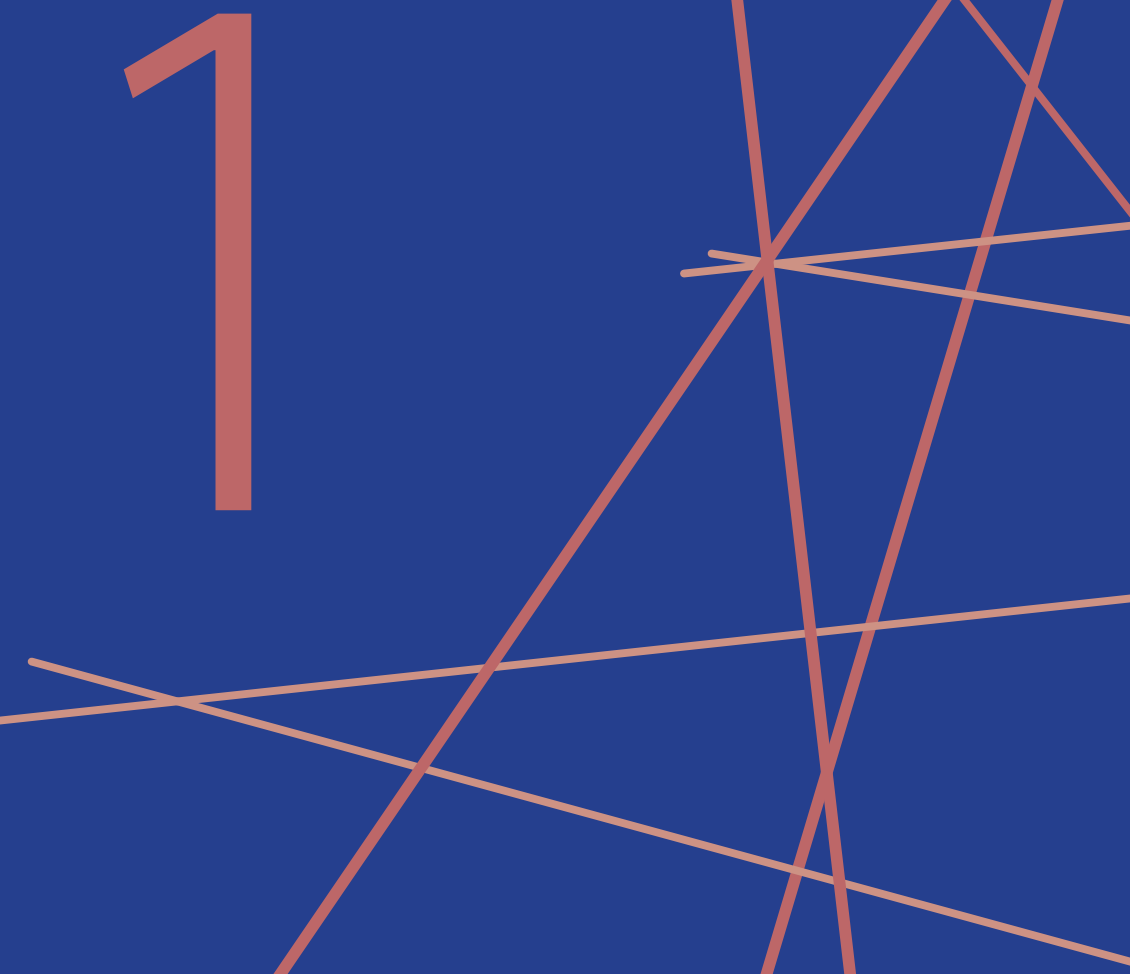


\section{Nuclear imaging}

Nuclear imaging makes an important contribution to medical imaging. It is aimed at functional imaging, as opposed to other commonly used imaging modalities like ultrasound, computed tomography (CT), and magnetic resonance imaging (MRI), which mainly focus on anatomical imaging. The workhorses in nuclear imaging are single photon emission computed tomography (SPECT) and positron emission tomography (PET). These are commonly coupled to a CT to form hybrid imaging systems (SPECT/CT and PET/CT). With these hybrid imaging systems, functional images can be fused with anatomical images. Whether to use SPECT or PET depends on the radionuclide that needs to be imaged. SPECT images single photons, or gamma radiation, whereas PET images positrons.

\section{SPECT}

A single photon emission computed tomography (SPECT) scanner detects gamma radiation by using gamma cameras (usually two, Figure 1.1). A gamma camera consists of a scintillation crystal and photo multiplier tubes (PMTs). The scintillation crystal will transform absorbed photons into light. The PMTs are able to amplify this very weak light signal into a measurable electronic signal. A gamma camera is able to detect a single photon, and to determine both its energy and location of absorption within the scintillation crystal. To create an actual image, a gamma camera needs to be mounted with a collimator [1]. A collimator is a lead sheet with hexagonal holes that only allows photons travelling parallel to the holes to pass (Figure 1.1). The detector head (gamma camera together with a collimator) acquires two-dimensional (2D) planar scintigraphy images. By rotating the detector heads around the patient (rotation takes $\sim 20$ minutes) and acquiring multiple $2 \mathrm{D}$ images from different angles, a three-dimensional (3D) image can be created (image reconstruction).

Collimators are designed for specific photon energies, and have a sensitivity and resolution depending on their septal thickness, hole length, and hole diameter [1]. The most commonly used collimator is the low-energy high-resolution (LEHR) collimator, which is designed for imaging of the most commonly used radionuclide for SPECT, technetium99m (99m Tc). ${ }^{99 m} \mathrm{mc}$ only emits gamma photons (140 keV), which makes it easy to image, and keeps the patient radiation dose low. Together with its half-life of 6 hours it is an ideal nuclide to study biological processes. ${ }^{99 \mathrm{mTC}}$ can be labelled to a variety of tracers and is used for diagnostic purposes in e.g. bone scans, myocardial perfusion scans, and sentinel node procedures [2-4].
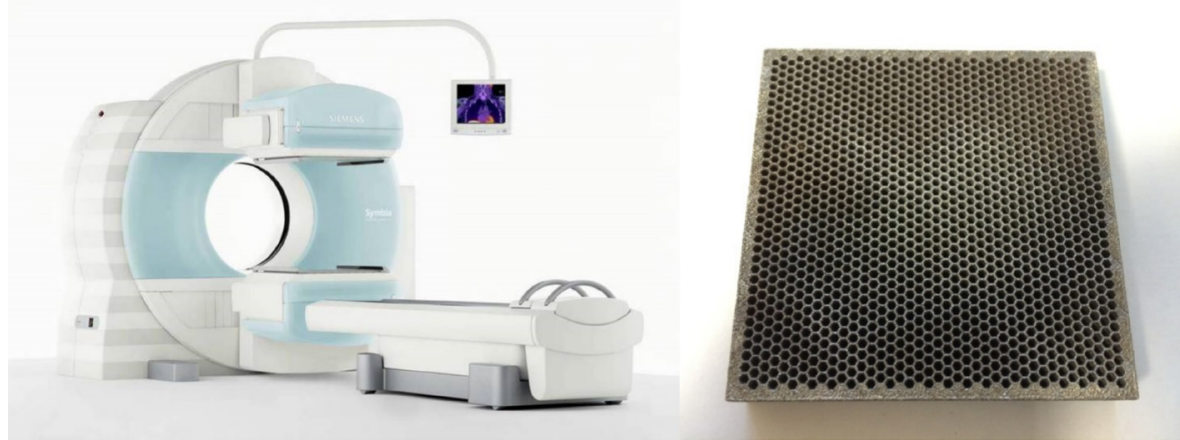

Figure 1.1: A single photon emission computed tomography / computed tomography (SPECT/CT) scanner, Siemens Symbia T (left) and a collimator (right).

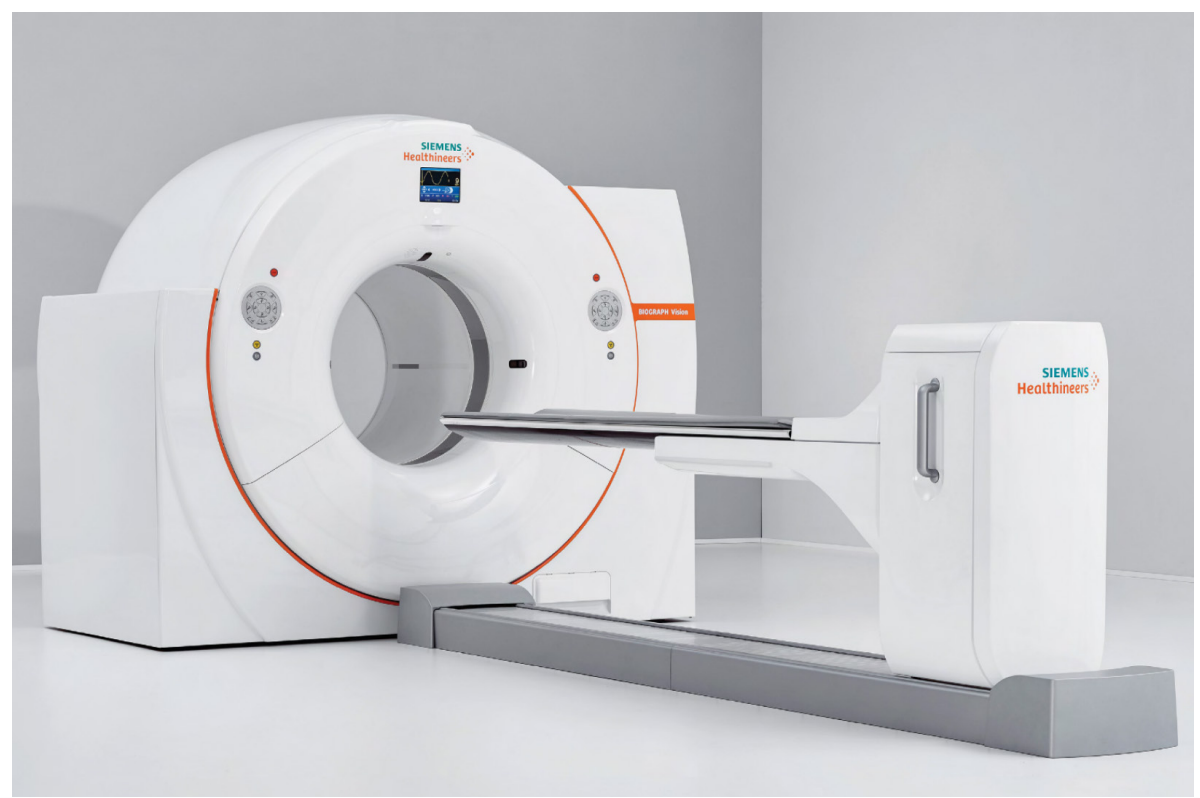

Figure 1.2: A positron emission tomography (PET) scanner, Siemens Biograph Vision.

\section{PET}

A positron emission tomography (PET) scanner detects positrons, although not directly (Figure 1.2). Annihilation of a positron creates a pair of photons (511 keV) that travel in opposite direction. These photons are detected by the PET scanner, in a similar way as the gamma camera detects single photons; by a scintillation crystal and PMTs (or silicon photomultipliers (SiPMs) for the newest generation of PET scanners). Because the PET scanner needs to detect photon pairs, it consists of detector rings, instead of detector 
heads like the SPECT scanner. Two photons are considered a pair when they are detected simultaneously, which in this case means less than about 5 nanoseconds apart. The photon pair can be connected by drawing a virtual line between the two detected photons, which is a so called line of response (LOR). The annihilation event occurred somewhere on this line. Today's PET scanners have a very good temporal resolution, which allows for measurement of the time difference by which the two photons were detected. This time of flight (TOF) information allows for specification of a region along the line of response where the annihilation event occurred [5]. By combining the information of all the measured LORs, a 3D image can be created (image reconstruction).

The most commonly imaged radionuclide for PET is fluor-18 $\left({ }^{18} \mathrm{~F}\right) .{ }^{18} \mathrm{~F}$ mainly emits positrons and can be labelled to, for example, fluorodeoxyglucose (FDG) for the detection and staging of tumours.

\section{Image reconstruction}

Creating a 3D image out of planar images (SPECT) or out of lines of response (PET) is called image reconstruction. For both SPECT and PET, iterative reconstruction methods like the ordered subsets expectation-maximisation (OSEM) method are used [6]. The idea of an iterative reconstruction method is that the reconstructed image is updated, and improved, in every iteration. Within the reconstruction method all sorts of physical properties can be modelled to obtain the best image quality [6]. Those are, for example: (i) attenuation correction, where the acquired $\mathrm{CT}$ is used to generate an attenuation map of the anatomy of the patient to estimate how many photons were attenuated before they reached the detector [7]; (ii) resolution recovery which compensates for the fact that a point source is measured as a blurred blob, by modelling the intrinsic resolution of the system [8]; (iii) scatter correction tries to compensate for photons that did not travel in a straight line, by estimating them based on photons acquired in a lower energy window (SPECT), or by fitting the tails of the projection profiles (PET) $[9,10]$. PET reconstruction methods usually also include a random correction. Random coincidences are two photons that are labelled as a pair, but that did not originate from the same annihilation event. The amount of randoms can be estimated by delaying the signal of one of the detectors of a detector pair [11]. Reconstruction algorithms can be further improved by modelling the interactions of single photons, so called Monte Carlo-based reconstructions. For SPECT, this allows detailed modelling of the scatter component, and improves image quality compared with conventional energy window-based scatter correction [12-14].

\section{Radionuclide therapy}

The most common radionuclides for nuclear imaging are used for diagnostic purposes. They emit (mainly) gammas or positrons, which make them easy to detect by SPECT or PET respectively, and keep the patient radiation absorbed dose low. However, there are also radionuclides that mainly emit electrons, a type of ionizing radiation. These can be used to irradiate specifically targeted tissues, for example tumours, and are used with a therapeutic purpose. An example of such a radionuclide is yttrium-90 (90Y). Imaging of such a radionuclide is challenging and brings along problems that diagnostic radionuclides like ${ }^{99 m} \mathrm{TC}$ and ${ }^{18} \mathrm{~F}$ do not have.

\section{Yttrium-90 imaging}

Yttrium-90 ( ${ }^{90} \mathrm{Y}$ ) was originally imaged by SPECT, even though it does not emit gamma photons. However, electrons emitted by ${ }^{90} \mathrm{Y}$ will create Bremsstrahlung photons as they travel through tissue. These Bremsstrahlung photons can be detected by a SPECT scanner. The main challenge for imaging in this case is the continuous spectrum of Bremsstrahlung. Bremsstrahlung does not have a photopeak that can be used for imaging, nor an energy window that can be used for scatter estimation. This difficulty in distinguishing primary photons from scattered photons degrades the image quality substantially [15]. Furthermore, the higher energy photons within the ${ }^{90} \mathrm{Y}$ energy spectrum have a relatively high probability to penetrate the collimator septa, which will further degrade the image [16]. To be able to acquire good quality images of ${ }^{90} \mathrm{Y}$, dedicated reconstruction algorithms have been developed that can better model scatter and collimator effects $[17,18]$. These dedicated reconstruction algorithms, Monte Carlo-based reconstructions, improve image quality compared with the clinical reconstruction algorithms (Figure 1.3). The downside of the Monte Carlo-based reconstructions is that they require a lot of computing power. Creating a single image may take hours. This the main reason why these reconstructions are mainly used in a research setting and have not made it to clinical practice yet.

Recently, it has been shown that PET can also be used for ${ }^{90} Y$ imaging, by using the very small positron branching ratio of ${ }^{90}$ Y (Figure 1.3) $[19,20]$. The hypothetical advantage of PET over SPECT imaging is the higher resolution of PET. However, PET imaging of ${ }^{90} \mathrm{Y}$ remains challenging because of the low positron yield of ${ }^{90} Y$, and the very high random fraction of ${ }^{90} Y$ imaging.

\section{Radioembolization}

An example of radionuclide therapy is selective internal radiation therapy (SIRT), also known as radioembolization. This is a minimally invasive treatment for liver malignancies. During radioembolization treatment, microspheres are injected in (a branch of) the hepatic artery and will preferentially lodge in and around the tumour(s). This preference is caused 
by the dual blood supply of the liver. The healthy liver parenchyma receives its blood from the portal vein and the hepatic artery. However, most hepatic malignancies solely rely on the hepatic artery for their blood supply. This leads to a preferential blood flow from the arteries to the tumour(s). When the microspheres are administered and lodge in the smaller blood vessels, the radionuclides that are trapped within the microspheres will decay and thereby emit beta-particles that locally irradiate the tumour(s).

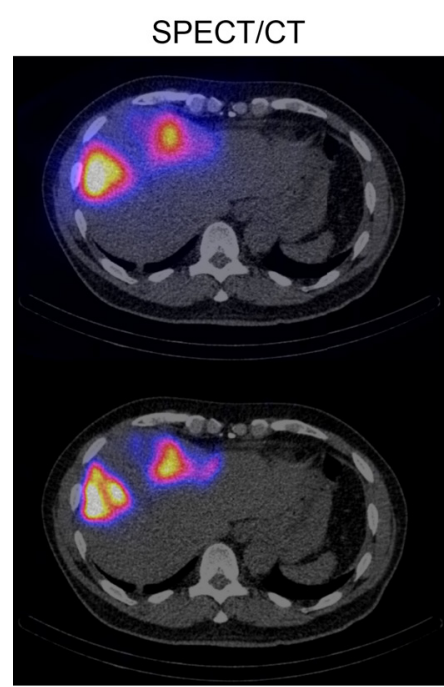

$\mathrm{PET} / \mathrm{CT}$

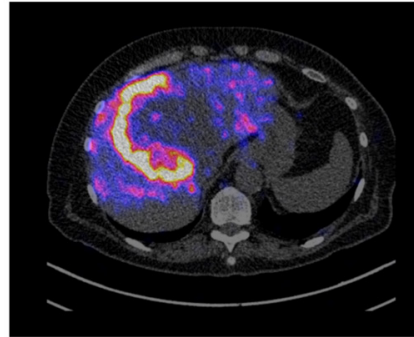

Figure 1.3: $S P E C T / C T$ (left) and $P E T / C T$ (right) of yttrium-90 (90Y), with the $C T$ image in greyscale and the better scatter correction than low activiy concention than the cinical SPECT in Costructed in the ribs, whereas in the Monte Carlo 90Y SPECT image al activity is nicely reconstructed within the liver.

\section{Radioembolization procedure}

Radioembolization is a complex, image-guided, minimally invasive procedure that involves multiple medical specialists and multiple imaging modalities. Patients are selected for radioembolization based on liver function (e.g. Child Pugh score), general performance status (e.g. Eastern Cooperative Oncology Group performance score), arterial blood flow (e.g. contrast enhanced CT) and tumour spread (e.g. ${ }^{18} \mathrm{~F}-\mathrm{FDG}$ PET). A radioembolization procedure consists of various steps that are shown in Figure 1.4

Patients considered for radioembolization will first undergo a pre-treatment procedure. This pre-treatment procedure starts in the intervention room. Here, an interventional radiologist will insert a microcatheter into the hepatic artery, guided by fluoroscopic $x$-ray images acquired by a c-arm (Figure 1.5). The blood supply of the liver is visualised and the injection position(s) for the treatment are determined. The exact location of the injection position(s) are recorded and a total of $150 \mathrm{MBq}{ }^{99 \mathrm{~m} T c}$ labelled macro-aggregated albumin (99mTc-MAA) is administered.

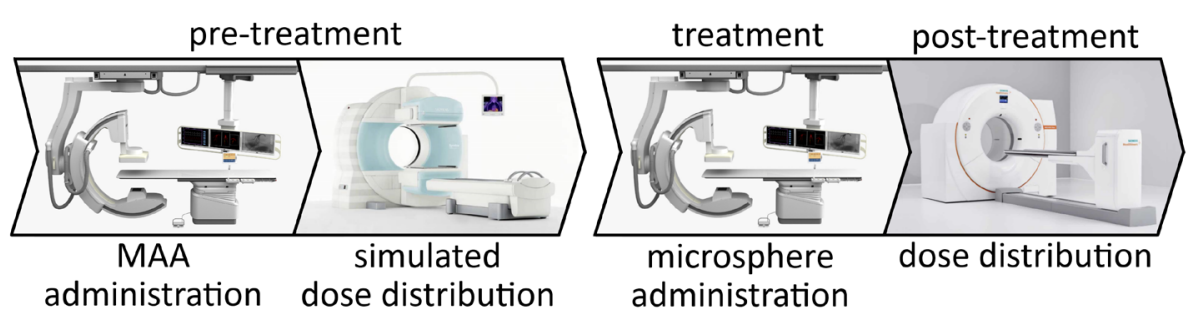

Figure 1.4: Steps in a radioembolization treatment. Pre-treatment procedure consisting of macroaggregated albumin (MAA) administration under fluoroscopic guidance (c-arm) followed by SPECT/CT to analyse the simulated dose distribution. During treatment the microspheres are administered, which is followed by a PET/CT (or SPECT/CT) to analyse the dose distribution.

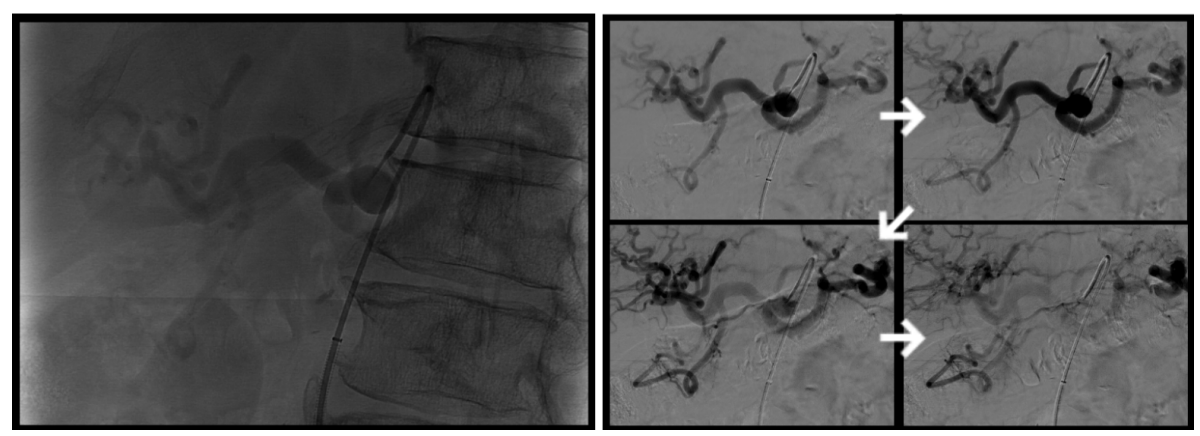

Figure 1.5: Fluoroscopic $x$-ray image where the microcatheter and part of the spine are visible (left), and four digital subtraction angiography (DSA) images (right). DSA images are produced by acquiring fluoroscopic images while injecting a contrast agent, and subtracting a pre-contrast image from all the acquired postcontrast images. The contrast agent highlights the vascular structure and shows the blood flow. The white arrows indicate the time line.

Macro-aggregated albumin (MAA) is a protein with, very roughly, the same size as the microspheres that are used for the treatment. The MAA particles are used to simulate the microsphere distribution. Labelling MAA with ${ }^{99 m} \mathrm{Tc}$ enables nuclear imaging of the simulated dose distribution in a relatively harmless way. For nuclear imaging, the patient is moved from the intervention room to the nuclear medicine department to acquire a SPECT/CT scan of the liver and planar scintigraphy images of the liver and the lungs. SPECT/CT is used to exclude any extrahepatic depositions and to check the intrahepatic dose distribution. The planar images are used to determine the amount of activity 
shunting to the lungs, the lung shunt fraction (LSF) (Figure 1.6). Based on the presence or absence of extrahepatic depositions, the LSF, and the tumour targeting, a nuclear medicine physician decides whether the radioembolization treatment can be performed safely, and how much activity will be administered during the treatment.

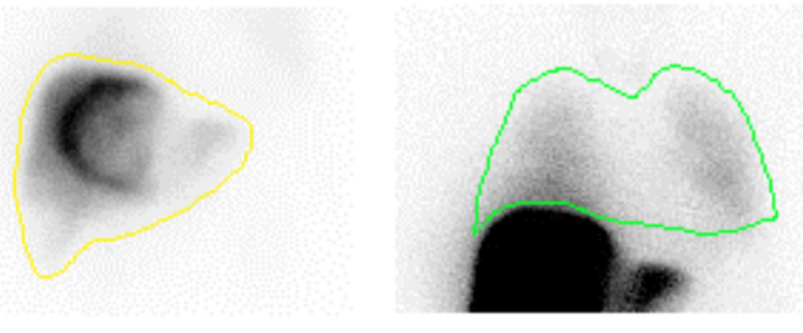

Figure 1.6: Planar scintigraphy images of 99mTC-MAA used to calculate the lung shunt fraction (LSF). The liver with its delineation (yellow) is shown on the left, and the lungs with its delineation (green) are shown on the right.

The treatment is usually performed about two weeks after the pre-treatment procedure. Microspheres are ideally administered at the exact location that was determined during the pre-treatment procedure. There are three types of microspheres that can be used for radioembolization treatment: ${ }^{00} \mathrm{Y}$ glass microspheres (TheraSpheres, BTG), ${ }^{90} \mathrm{Y}$ resin microspheres (SIR-spheres, Sirtex Medical), and holmium-166 ( $\left.{ }^{166} \mathrm{Ho}\right)$ microspheres (QuiremSpheres, Quirem Medical B.V.). These microspheres differ in size distribution and specific activity (Table 1.1). After treatment, the patient will receive a SPECT/CT $\left({ }^{166} \mathrm{Ho}\right.$ microspheres) or a positron emission tomography / computed tomography (PET/CT) ${ }^{90} \mathrm{Y}$ glass/resin microspheres) scan to confirm absence of extrahepatic depositions and treatment of all targeted tumours.

Table 1.1: Characteristics of the three types of microspheres used for radioembolization.

\begin{tabular}{lll}
\hline Microsphere & size & Specific activity \\
\hline${ }^{90}$ glass & Mean: $25 \mu \mathrm{m}[21]$ Range: $20-30 \mu \mathrm{m}[22]$ & $\sim 2500 \mathrm{~Bq} /$ sphere[22] \\
${ }^{90}$ resin & Mean: $32 \mu \mathrm{m}[21]$ Range: $20-60 \mu \mathrm{m}[23]$ & $\sim 50 \mathrm{~Bq} / \mathrm{sphere}[23]$ \\
${ }^{166 \mathrm{Ho}}$ & Mean: $30 \mu \mathrm{m}[24]$ Range: $15-60 \mu \mathrm{m}[24]$ & $\sim 240-375 \mathrm{~Bq} / \mathrm{sphere[24]}$ \\
\hline
\end{tabular}

\section{Prescribed activity calculation}

Radioembolization treatment planning is based on the pre-treatment procedure. In absence of extrahepatic depositions, the prescribed activity is limited by the radiation dose in the lungs and in the healthy liver parenchyma. The recommendation for ${ }^{90} Y$ glass microspheres and ${ }^{166} \mathrm{Ho}$ microspheres is to not exceed a predicted lung dose of $30 \mathrm{~Gy}$ $[24,25]$. The predicted lung dose is calculated using the lung shunt fraction (LSF), which is based on planar scintigraphy (Figure 1.6), and the lung mass, which is usually estimated to be $1 \mathrm{~kg}$. The manufacturer of ${ }^{90} \mathrm{Y}$ resin microspheres recommends to reduce the amount of prescribed activity by $20 \%$ in case of an LSF between $10 \%$ to $15 \%$, by $40 \%$ in case of an LSF between $15 \%$ to $20 \%$, and to not proceed with the treatment in case of an LSF over $20 \%$.

To calculate the prescribed activity for radioembolization treatment, the manufacturers of ${ }^{90} \mathrm{Y}$ glass microspheres and ${ }^{166} \mathrm{Ho}$ microspheres use the mono-compartment model, where the prescribed activity is calculated as:

$$
A(G B q)=\frac{D(G y) * M_{\text {target }}(k g)}{C(J / G B q)}
$$

Here, D is the desired dose in Gy, which is 60 Gy for ${ }^{166} \mathrm{Ho}$ microspheres, and 80-150 Gy for ${ }^{90} Y$ glass microspheres [24-28]. $M_{\text {target }}$ is the mass of the targeted tissue in $\mathrm{kg}$. This can be either the mass of the entire liver, or the mass of the treated liver segment(s), depending on tumour location(s). C is a constant depending on the radionuclide used, and describes the deposited energy with the assumption that all radiation is locally absorbed. C is $50 \mathrm{~J} /$ GBq for ${ }^{90} \mathrm{Y}$, and $15.9 \mathrm{~J} / \mathrm{GBq}$ for ${ }^{166} \mathrm{Ho}[29,30]$.

The manufacturer of ${ }^{90} \mathrm{Y}$ resin spheres provides three methods to calculate the prescribed activity for their European customers (for their American customers they solely recommend the BSA-method in combination with limiting the lung dose to <30Gy). The first one is the empirical model, where the prescribed activity is dependent on the percentage of tumour involvement. The recommended prescribed activity is $3.0 \mathrm{GBq}$ for tumour involvement over 50\%, $2.5 \mathrm{GBq}$ for tumour involvement between $25 \%-50 \%$, and $2.0 \mathrm{GBq}$ for tumour involvement below $25 \%$. However, this method does not take the liver volume into account and is considered obsolete because of the unfavourable toxicity profile [31]. The second, and most widely used, method for resin microspheres, is the Body Surface Area (BSA) method, were the prescribed activity is calculated as:

$$
A(G B q)=(B S A-0.2)+\frac{V_{\text {tumour }}}{V_{\text {tumour }}+V_{\text {liver }}}
$$

Here, BSA is the body surface area, calculated from the patient's height and weight. $V_{\text {tumour }}$ is the volume of the tumour and $V_{\text {liver }}$ is the volume of the normal liver tissue, both calculated from a CT scan. The third method is the partition model, where the prescribed activity is calculated as: 
$D(G y) *\left(T / N_{N} * M_{\text {tumour }}(k g)+M_{\text {liver }}(k g)\right)$

$A(G B q)=$ $49.760 *(1-L S F)$

Here, $\mathrm{D}$ is the maximum tolerable dose to the normal liver [23]. T/N is the average tumour to normal liver activity concentration ratio determined from ${ }^{99 \mathrm{mTC}-M A A ~ S P E C T / C T, ~} \mathrm{M}_{\text {tumour }}$ the mass of the tumour in $\mathrm{kg}, \mathrm{M}_{\text {liver }}$ the mass of the normal liver parenchyma in $\mathrm{kg}$, and LSF is the lung shunt fraction. The partition model is the only model taking the differences in activity uptake by the tumour and healthy liver parenchyma into account. However, the required average T/N can pose a problem in poorly defined tumours, or in patients with multiple tumours with different uptake. Furthermore, segmentation of all structures is labour-intensive, which makes this method less attractive in daily practice.

Radioembolization can be used to treat the whole liver, or to treat specific liver segments, For ${ }^{90} \mathrm{Y}$ glass and ${ }^{166} \mathrm{Ho}$ microspheres, the mono-compartment model is used with a target mass, which is either the mass of the whole liver, or the mass of the specific liver segments to be treated. For ${ }^{90} Y$ resin microspheres, the most widely used planning method, the BSAmethod, is designed to calculate a prescribed activity for a radioembolization treatment of the whole liver. If a partial liver treatment is performed with ${ }^{90} Y$ resin microspheres, a correction factor needs to be applied. When, for example, only $40 \%$ of the liver is treated, only $40 \%$ of the originally calculated prescribed activity should be used. The same principle holds for radioembolization treatments consisting of multiple injection sites. For every injection site, the corresponding treated liver volume is determined (based on contrastenhanced (T), and the corresponding prescribed activity is calculated.

\section{Imaging to improve radioembolization}

Since radioembolization is an image-guided procedure and multiple imaging modalities are involved in the treatment, there is a potential large gain to be made in treatment efficiency and outcome by improving imaging techniques.

At the moment, imaging in the intervention room is limited to the c-arm, which is mainly used for positioning of the catheter and does not give feedback on the administered dose. Efforts to allow real time nuclear and anatomical imaging in the intervention room are being made [32]. This would enable dosimetry during the treatment, and could guide administration of the microspheres. The ultimate goal of such a hybrid imaging modality would be to perform radioembolization in a single-session; the pre-treatment procedure is than directly followed by the treatment procedure. This could result in less burden to the patient and the catheter could stay in place for the treatment procedure, because the patient does not have to be moved to the nuclear medicine department. Repositioning of the catheter can be difficult, and the reproducibility of the catheter tip position is critical for the predictive value of the pre-treatment procedure [33-36]. Radioembolization treatment performed in a single-session could therefore also improve the reliability of the pre-treatment procedure

Apart from introducing a new imaging modality, radioembolization can also be improved by improving current imaging modalities. First of all, pre-treatment imaging can be improved. In current clinical practice, the lung shunt fraction (LSF) is based on planar images of the lungs and the liver. However, these planar images are known to overestimate the LSF [37-40]. This is caused by the absence of accurate scatter and attenuation correction. Scatter and attenuation correction can be better incorporated in SPECT/CT image reconstruction, and using these instead of planar ${ }^{99 \mathrm{~m} T \mathrm{~T}-M A A}$ images to estimate the LSF, reduces the overestimation [40]. When calculating the lung dose, another factor introducing errors is the standard lung mass of $1 \mathrm{~kg}$ that is used for all patients. A more patient specific lung mass can be estimated from a diagnostic CT [41].

Another important factor influencing the LSF and lung dose estimations is the difference between the MAA particles used for the pre-treatment procedure and the microspheres used for the treatment. These particles differ in shape and size distribution, which can lead to differences in dose distribution $[35,36,42]$. Furthermore, MAA particles break down into smaller particles, and ultimately into pertechnetate. These smaller particles are more likely to shunt to the lungs and thereby increase the LSF $[43,44]$. This effect can be minimized by keeping the time between injection of the ${ }^{99 \mathrm{mTC}} \mathrm{TMAA}$ and the scanning as short as possible [43]. When radioembolization is performed with ${ }^{160} \mathrm{Ho}$ microspheres, it is possible to perform the pre-treatment procedure with the same particles as the treatment, ${ }^{166} \mathrm{Ho}$ microspheres in a lower dosage. Using the same particle leads to a more accurate estimation of the lung dose [39].

In addition to the estimated lung dose, the estimated intrahepatic dose distribution affects treatment planning. This is most obvious when the partition model is used for the treatment planning, because in this case the tumour to non-tumour activity concentration ratio (T/N) directly influences the prescribed activity. In case of the ${ }^{90} \mathrm{Y}$ glass microspheres, the desired dose (80-150 Gy) is chosen by the treating physician. At the UMC Utrecht, a higher desired dose will be chosen for patients that show good tumour targeting on pre-treatment imaging than for patients that show poor or moderate tumour targeting. However, the predictive value of the pre-treatment procedure with MAA particles for the intrahepatic dose distribution is debatable $[33-35,45,46]$. Again, the difference between the MAA particles and the microspheres are mentioned as a cause $[35,36,47]$. Using the same particle for pre-treatment and treatment $\left({ }^{166} \mathrm{Ho}\right.$ microspheres), leads to a higher 
Post-treatment imaging also plays a major role in the improvement of radioembolization, although it is not part of standard clinical care in all centres. First of all, post-treatment imaging can reveal whether all tumours received the planned radiation dose or that a patient might benefit from a second treatment. Furthermore, post-treatment imaging allows dose-response and dose-toxicity relations to be established. These relations can be included in the prescribed activity calculation models, to make treatment planning more personalised [38].

\section{Outline thesis}

The broad aim of this thesis is to investigate nuclear imaging techniques that can improve treatment planning in radioembolization. To put this into perspective, advances in imaging techniques for radionuclide therapy are discussed in Chapter $\mathbf{2}$. An effort to allow nuclear imaging in the intervention room, by combining a c-arm with a gamma camera for simultaneous fluoroscopic and nuclear imaging, is discussed in Chapter 3. Chapter 4 will focus on the differences between the three microspheres. Using the same particle for the pre-treatment procedure and the treatment procedure, as can be done for the ${ }^{166} \mathrm{Ho}$ microspheres, might also be possible for ${ }^{90} \mathrm{Y}$ microspheres. This introduces some challenges regarding imaging. The possibility of estimating the lung shunt fraction based on pre-treatment ${ }^{90} \mathrm{Y} \mathrm{SPECT/CT} \mathrm{and} \mathrm{PET/CT} \mathrm{is} \mathrm{investigated}$ in Chapter 5. This idea of ${ }^{90} \mathrm{Y}$ pre-treatment imaging is expanded in Chapter $\mathbf{6}$ to the detection of extrahepatic depositions and the quantification of intrahepatic dose distribution. In Chapter $\mathbf{7}$ the quantitative accuracy of a 'new generation' PET/CT scanner for ${ }^{90} Y$ imaging is investigated. The thesis is concluded with a general discussion

in Chapter 8 and summarised in Chapter $\mathbf{9}$ 


\section{Chapter 2}

Technical advances in image guidance of radionuclide therapy 


\section{Abstract}

Internal radiation therapy with radionuclides (i.e., radionuclide therapy) owes its success to the many advantages over other, more conventional, treatment options. One distinct advantage of radionuclide therapies is the potential to use (part of) the emitted radiation for imaging of the radionuclide distribution. The combination of diagnostic and therapeutic properties in a set of matched radiopharmaceuticals (sometimes combined in a single radiopharmaceutical) is often referred to as theranostics, and allows accurate diagnostic imaging before therapy. The use of imaging benefits treatment planning, dosimetry, and assessment of treatment response. This paper focuses on a selection of advances in imaging technology relevant for image guidance of radionuclide therapy. This involves developments in nuclear imaging modalities, as well as other anatomic and functional imaging modalities. The quality and quantitative accuracy of images used for guidance of radionuclide therapy is continuously being improved, which in turn may improve the therapeutic outcome and efficiency of radionuclide therapies.

\section{Introduction}

Radionuclide therapy combines aspects of both chemotherapy (such as the cancer cell targeting capabilities) and external-beam radiotherapy (by making use of ionizing radiation). An advantage of using radionuclide therapy over external beam radiotherapy is that radionuclide therapy has the potential to eliminate not only the primary tumour, but also metastatic or undetected tumours. Moreover, unlike chemotherapy, the radiopharmaceuticals used for radionuclide therapy potentially destroy adjacent tumour cells even if they lack specific uptake. Strategies for targeted delivery of radionuclides include binding of radionuclides to a cell targeting molecule, such as treatment of neuroendocrine tumours with ${ }^{177}$ Lu-DOTATATE [48]; use of the ability of the radionuclide to target specific cells on its own, such as the treatment of thyroid carcinoma with ${ }^{131}$ | [49]; and intra-arterial injection, such as radioembolization with ${ }^{90} \mathrm{Y}$ microspheres for liver malignancies [50].

Another advantage of radionuclide therapies is the potential to use the emitted radiation for imaging of the radionuclide distribution. The combination of diagnostic and therapeutic properties in a set of matched radiopharmaceuticals (sometimes combined in a single radiopharmaceutical) is often referred to as theranostics, and allows accurate diagnostic imaging before therapy. The use of imaging benefits treatment planning, dosimetry, and assessment of treatment response. Therefore, developments in imaging technologies may also benefit the efficacy of radionuclide therapies. This paper focuses on the technical developments in imaging technology relevant for imaging of radionuclide therapy. Developments in the field of the traditional nuclear imaging modalities (i.e., scintigraphy, SPECT and PET), as well as other anatomic and functional imaging modalities, are discussed.

\section{SPECT and scintigraphy}

The type of decay of radionuclides used for nuclear medicine therapy or its guidance determines what modality is used for imaging. Planar scintigraphy or SPECT is used for imaging of radionuclides such as ${ }^{177} \mathrm{Lu},{ }^{90} \mathrm{Y}$, and ${ }^{1{ }^{13}} \mathrm{l}$, that are used for radionuclide therapy. These nuclides emit $\gamma$-photons (or Bremsstrahlung photons), which can be imaged with a $y$-camera.

\section{Current status}

Modern SPECT/CT systems can be used for planar as well as tomographic imaging. Planar imaging is readily used to acquire whole-body images in a limited amount of time, because imaging the whole body with a tomographic (SPECT) method would be time-consuming. SPECT is used to acquire 3-dimensional data to discern structures that 
would overlap one another on planar images. Planar and tomographic images are often acquired in sequence to obtain whole-body images in addition to 3-dimensional images of a specific part of the body.

Quantitative SPECT images can be obtained by converting the acquired counts into a distribution of absorbed dose (in Gy), which can benefit planning and dosimetry of radionuclide therapy. However, because of effects such as photon attenuation, scatter, and blurring, SPECT/CT images are not inherently quantitative. Attenuation correction is commonly incorporated in the projection operators of the iterative reconstruction method using attenuation maps from co-registered CT images. Scatter correction is also routinely implemented into clinical practice and is often performed using the triple energy window method [51]. Image quality can be further improved by applying resolution recovery. This is done by accurately characterising the shape of the point spread function which depends on the distance from the camera, and is rotationally variant because of the hexagonal pattern of the collimator septa. The point spread function model can subsequently be incorporated into the reconstruction algorithm [52].

Even though image-degrading effects such as attenuation, scatter, and blurring can be partially corrected, SPECT images suffer from partial-volume effects and quantification errors.

\section{Advances in SPECT and scintigraphy}

This section discusses SPECT developments improving image quality in general, as well as those improving guidance of radionuclide therapy.

Instrumentation: Collimators and detectors. Tumour dosimetry requires accurate quantification of small structures. This is severely hampered by the partial-volume effects that, by limiting image resolution, reduce contrast. Because image resolution is largely determined by collimator resolution, development of collimators that are less easily penetrated is one strategy to reduce partial-volume effects. Because many of the isotopes used for radionuclide therapies (e.g., ${ }^{131}$ I and ${ }^{90} \mathrm{Y}$ ) emit high-energy photons that easily penetrate the collimator septa and reduce image resolution, optimising the collimator may be crucial to achieving quantitative accuracy. The strategy used to optimise parallel-hole collimators for high-energy applications, is to increase septal thickness and septal length to limit septal penetration. However, optimising the design of a parallelhole collimator involves a trade-off between septal penetration, spatial resolution, and sensitivity. Alternatively, one can use pinhole collimators designed to limit collimator penetration [53]. However, quantitative pinhole SPECT remains a challenge in clinical practice for the following reasons: (i) truncated projections may lead to reconstruction artefacts, (ii) dedicated software may be required for quantitative imaging, and (ii) the sensitivity of pinhole collimators is limited. Multiple pinholes can be used to overcome the problem of limited sensitivity [54]. Image quality may also be improved using a collimator consisting of cone-shaped holes (the parallel-cone collimator; Figure 2.1), which limits collimator penetration while preserving resolution and sensitivity [55]
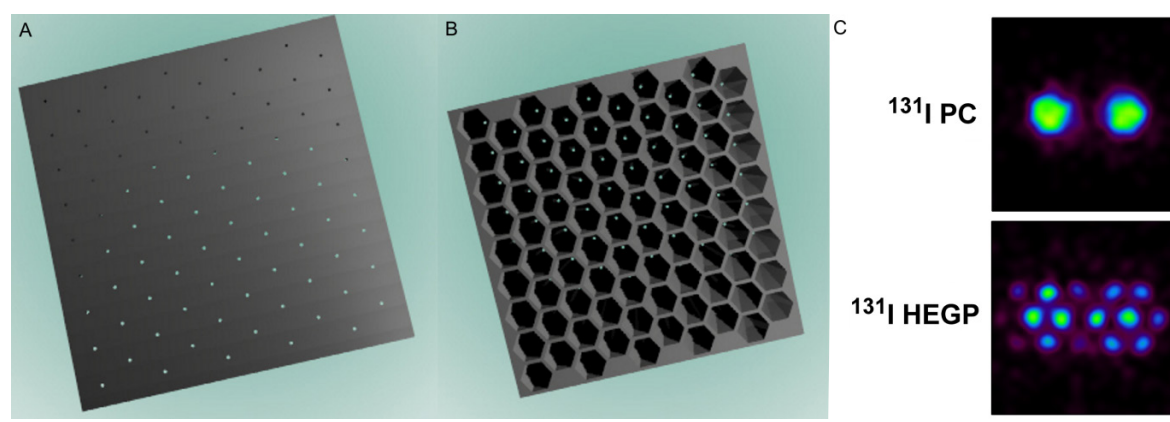

Figure 2.1: Face (A) and back (B) of parallel-cone (PC) collimator. (C) Double-point-source Monte Carlo simulations obtained for ${ }^{13} \mid$ on PC collimator and high-energy general-purpose (HEGP) collimator. PC collimator can detect the two sources separately, whereas HEGP collimator cannot. (Reprinted from Beijst et al.[55])

Detectors can be optimised for high-energy photons (emitted by the isotopes used for radionuclide therapies) using thicker crystals (5/8 inch instead of the standard 3/8 inch), or using scintillation crystals with high stopping power, which increases the detection efficiency. Walrand et al. have designed a camera dedicated to Bremsstrahlung imaging of ${ }^{90} \mathrm{Y}$ [53]. They describe a dedicated system with a $30 \mathrm{~mm}$ thick bismuth germanate crystal and an $8 \mathrm{~mm}$ high-energy pinhole collimator with extra shielding to prevent penetration of high-energy photons. Additionally, it is suggested that the camera can be mounted on a robotic gantry for use in the intervention room, as shown in Figure 2.2.

Reconstruction algorithms. For the application of radionuclide therapy, quantitative results benefit dosimetry and assessment of treatment response. However, iterative reconstruction algorithms are able to obtain images with a high signal-to-noise ratio, but do not necessarily generate (linearly) quantitative images. In general, SPECT images have long been regarded as solely qualitative images, in contrast to PET, which allows the use of semi-quantitative or quantitative measures such as the standard uptake value (SUV). Despite the relatively low resolution of SPECT images (as compared with PET), quantitative SPECT images can now be obtained when image-degrading effects are adequately corrected. These corrections include those for attenuation, scatter, point spread function, or dead time. Although quantitative images can be obtained using easy-to-implement scatter correction techniques, the accuracy of quantitation can be improved using Monte 
Carlo-based scatter modelling [60]. Monte Carlo-simulated forward projections can be used in the reconstruction algorithm to accurately model object scatter and, hence, correct for the image-degrading effects of scatter.
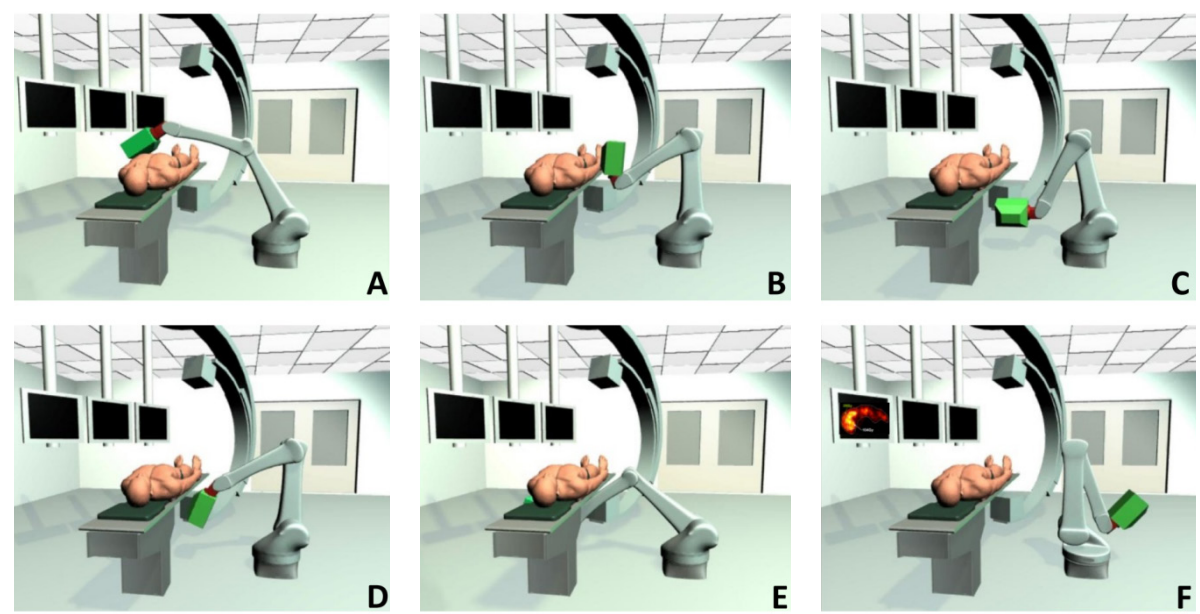

Figure 2.2: Interventional multi-pinhole SPECT camera mounted on 6-axis arm robot [56]. The images show how the robotic gantry is able to acquire tomographic images from multiple angles.

Advances in detector technology also include the development of solid-state detectors [57]. Cadmium zinc telluride (CZT) detectors directly convert the energy of incident $\gamma$-photons into an electric signal, in contrast to indirect scintillation-based detector systems that require photomultiplier tubes. Advantages of CZT detectors are the superior energy resolution and the high counting rate capability as compared with photomultiplier cameras with sodium iodide (Nal) crystals. Superior energy resolution results in a lower detection of scattered events due to a narrow energy window. This advantage also paves the way for dual-isotope applications, for which clear separation of photopeaks is essential [58]. The fact that CZT detectors can detect high count rates is an advantage for radionuclide therapies, as high activities are generally used to deliver the therapeutic radiation dose. However, CZT detectors have low stopping power, which makes them less suitable for imaging of high-energy photons. CZT technology is more suitable for isotopes with low-energy photopeaks, such as ${ }^{99 \mathrm{~m} T C},{ }^{166} \mathrm{Ho}$, or ${ }^{177} \mathrm{Lu}[59]$

Over the years, several Monte Carlo codes have been incorporated into the reconstruction algorithm, such as GATE, SIMSET, MCNP, SIMIND, and UMCS [61]. Because Monte Carlo simulations are notoriously slow, acceleration is often performed using variance reduction techniques. One of the strategies is to use convolution-forced detection, which forces particles toward the collimator by creating daughter particles at every interaction in the phantom with weighting to correct for the likelihood of detection [12,13]. Despite the challenges of quantitative SPECT, a quantitative accuracy of within $5 \%$ can be obtained, which is equivalent to the accuracy of PET/CT systems [62].

Quantitative images may be used for dosimetric applications in radionuclide therapy. Obtaining these images is not straightforward, because the acquired projection images are measured in counts. These can be converted into quantitative images representing the activity concentration (in Bq/unit volume) using quantitative reconstruction algorithms. In turn, quantitative images that represent patient dose (in Gy) may be obtained by taking into account an estimation of the retention time, the specific energy released and absorbed, and information about the spatial dose distribution (e.g., dose point kernels). The retention time may be estimated by assuming a theoretic effective half-life, or by acquiring multiple scans over time to achieve a time-integrated activity in a target volume (organ or tumour). Subsequently, the absorbed dose can be calculated by multiplying the activity concentration by a radionuclide-specific constant [63], by convolving the activity distribution with a dose point kernel, or by performing Monte Carlo simulations [64], depending on the required accuracy of the dose estimation.

Advances in the development of SPECT reconstruction algorithms also involve the use of anatomic priors. For example, maximum a posteriori algorithms that incorporate smoothing within, but not across, organ or lesion boundaries may improve the quality of images used for guidance of radionuclide therapy [65]. A fundamental issue with anatomic priors is that the resulting images are no longer purely molecular, but also include anatomic information

Developments in SPECT reconstruction algorithms also include corrections for cardiac or respiratory motion [66]. The correction for cardiac motion mostly benefits cardiac applications, whereas corrections for respiratory motion may improve lesion detection in general. Motion correction is discussed in more detail in the next section.

\section{PET}

${ }^{18}$ F-FDG PET accounts for most PET studies in clinical practice and is often used for staging and follow-up after radionuclide therapy. However, PET is also used for imaging of other nuclides (e.g., ${ }^{90} \mathrm{Y},{ }^{124},{ }^{64} \mathrm{Cu}$, and ${ }^{68} \mathrm{Ga}$ ) for treatment planning, dosimetry, and assessment of treatment response in radionuclide therapies. In all cases, good quantitative accuracy is required, either for precise SUV- based therapy response monitoring, or more detailed dosimetry for therapy planning. 


\section{Current status}

Like SPECT, quantitative PET requires application of correction techniques. Attenuation correction for PET can easily be performed by determining the attenuation correction sinogram, typically based on co-registered CT data. In addition, scatter correction is often implemented in clinical practice using the single-scatter simulation method [67]. Correction for random counts is generally performed by applying delayed-event subtraction [68].

The time difference in detection between annihilation photons provides information about the location of the annihilation event along the line of response. This time-of-flight (TOF) information can be incorporated in the reconstruction during the backprojection step to improve image quality. The availability of TOF information has paved the way for imaging isotopes with very low positron abundance, such as ${ }^{90} \mathrm{Y}$.

Because the intrinsic resolution of PET detectors is limited, the shape of the point spread function can be incorporated in the reconstruction method to improve the quality of reconstructed images. This process is often referred to as resolution recovery.

Radiation detection systems suffer from dead-time effects due to pulse pile-up when subjected to high counting rates. Dead-time losses are routinely corrected for, based on measurements of a large range of activities and knowledge of the true counting rate model

\section{Advances in PET}

The quality of PET images is continuously being improved by advances in PET instrumentation and reconstruction aimed at increasing resolution and sensitivity, which in turn allows a more accurate determination of the SUV. Using nuclides such as ${ }^{124}$ and ${ }^{90} Y$, such improvements may aid in optimised targeting, treatment planning, dosimetry, and assessment of treatment response in radionuclide therapies.

Instrumentation. PET scanners traditionally use photomultiplier tubes to convert the optical signal coming from the scintillation crystal into an electronic signal. However, semiconductor-based alternatives are also becoming commercially available for clinical systems. The most commonly used alternatives are avalanche photodiodes and silicon photomultipliers (SiPMs). Avalanche photodiodes are essentially semiconductor photodetectors that operate in avalanche mode, which means that the output is linear with the amount of scintillation light. SiPMs consist of a multi-pixel array of small avalanche photodiodes that operate in Geiger mode. Therefore, the output of a single pixel of an SiPM is not linear with the amount of scintillation light. However, the number of SiPM pixels that produce an avalanche pulse is a measure of the energy of the incident $\gamma$-photon. The individual SiPM pixels have a size of 20-100 $\mu \mathrm{m}$. SiPMs can be fabricated to couple with smaller crystals and therefore improve resolution [69]. Another advantage of SiPMs is their good timing resolution, which improves the signal-to-noise ratio of PET images through better time-of-flight estimation $[70,71]$.

Increasing the timing resolution enhances the time-of-flight estimation and, consequently, the signal-to-noise ratio of the images [70]. Therefore, improvement of the timing resolution is subject to research. Time-of-flight detectors typically use $4 \times 4 \times 22$ $\mathrm{mm}$ scintillation crystal elements, with photodetectors placed on the top of the crystal element for readout. Moses et al. developed a detector in which the photodetector is mounted on the side of the crystal element [72]. This design reduces the mean path length of the scintillation photons to the photodetectors, which in turn improves timing resolution. Another approach to improving the timing resolution is double-sided readout, as described by Seifert and Schaart [73]. They describe a setup in which photodetectors are mounted on the top and bottom of the crystal element, enabling correction of depthdependent effects to the timing uncertainty. An additional advantage of this approach is that depth of interaction can be extracted.

Another method to improve the efficiency of PET images is by increasing axial detector length. The image quality of low-abundance isotopes, such as ${ }^{90} \mathrm{Y}$, may benefit from this approach. Extending the axial field of view of a PET scanner from $16.2 \mathrm{~cm}$ (3 detector rings) to $21.8 \mathrm{~cm}$ (4 detector rings) already significantly increases system sensitivity [74]. A whole-body PET system with an axial detector length of approximately $2 \mathrm{~m}$ is currently under development at the University of California, Davis, and shown in Figure 2.3 [75]. Although the increase in axial detector length increases sensitivity, it also increases the adverse effects of parallax errors as observed with traditional detectors. Parallax errors occur when an annihilation photon enters one crystal element but is absorbed after penetrating an adjacent crystal element. This error generally occurs for high oblique angles of incidence. Parallax errors may be reduced if detectors are equipped with depthof-interaction information $[76,77]$.

Reconstruction. Advances in image reconstruction include modelling of motion by incorporating information on motion into the reconstruction algorithm $[78,79]$. Motion correction can easily be performed by including counts from only a certain phase of the respiratory cycle (e.g., end expiration). However, such a phase-gated approach suffers from prolonged scan times (or increased noise) because of loss of data that are discarded for reconstruction. Correction for respiratory motion may be implemented using information on motion from additional hardware (e.g., a belt system). However, similar results can also 
be obtained with data-driven approaches without the use of additional hardware [80]. Event-by-event motion correction is an approach in which all data are used to reconstruct an image $[81,82]$. This approach leads to less noise than phase-gated motion correction In general, reconstruction algorithms with motion correction often use data that were acquired in list mode. Acquiring data in list mode means that for each count the time and position of detection are stored. List-mode acquisitions are especially useful for motioncorrected reconstruction algorithms, because retrospective redefinition of phases in phase-gated sinograms is allowed.

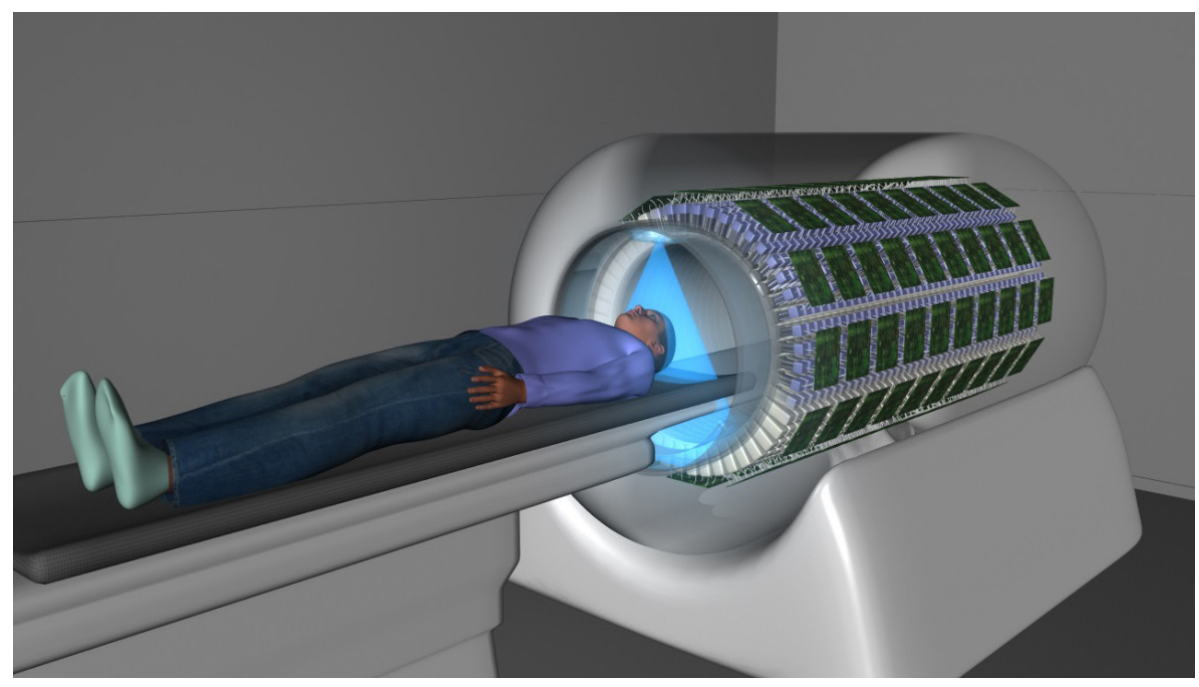

Figure 2.3: Explorer total-body PET scanner. (Courtesy of Drs. Simon R. Cherry and Ramsey D. Badaw University of California, Davis.)

Like SPECT images, PET images may be improved by integrating anatomic information in the reconstruction algorithm using anatomic priors $[83,84]$. In addition to using anatomic priors, smoothing priors can be incorporated with a penalty term that suppresses noise $[85,86]$

For some isotopes, positrons are emitted in cascade with the emission of $\gamma$-photons, so that additional photons are emitted together with the two annihilation photons (Figure 2.4). These additional photons are called prompt $Y$-photons. ${ }^{124}$ and ${ }^{86} Y$ are examples of isotopes that emit prompt $\gamma$-photons in cascade with the positron and are often used for matched-pairs dosimetry of the therapeutic isotopes ${ }^{131}$ and ${ }^{90} Y$, respectively [87]. When these prompt $\gamma$-photons are detected, they may be mistaken for an annihilation photon, resulting in an erroneous line of response. The adverse effects of the prompt $\gamma$-photons can be corrected for by implementing a prompt $\gamma$-correction in the reconstruction algorithm $[88,89]$
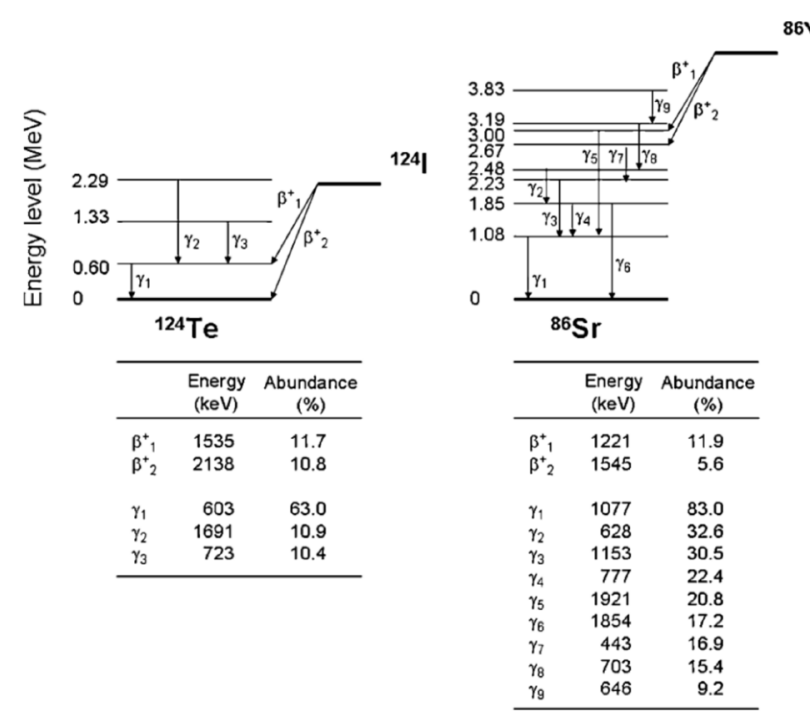

${ }^{86} Y$

Figure 2.4: Decay scheme of several isotopes that emit prompt $\psi$-rays in cascade with positron emission [90.

PET/MRI. PET/MRI has several advantages over PET/CT, of which higher soft tissue contrast is probably most important for treatment planning, dosimetry, and assessment of response to radionuclide therapies. Additionally, the simultaneous acquisition of co-registered MR images may benefit accurate dosimetry. Also, MRI can be used to identify organs at risk (or parts of one organ), which may be essential for determining the maximum tolerable radionuclide dosage. Moreover, the simultaneous acquisition of anatomic and molecular images enables accurate motion correction, for instance, by making real-time MR images of the lungs.

However, the integration of PET and MRI modalities does bring about serious challenges, because mutual interference between modalities can occur. That is, photomultiplier tubes used in traditional PET detectors do not function in the strong magnetic fields used for MRI. Moreover, the radiofrequency signal used for MRI is affected by the PET modules [91]. For this reason, the first generation of PET/MRI systems acquired PET and MR images in sequence and the modalities were spatially separated. Integrated systems in which the PET detectors are inserted into the bore of the MR scanner are now available so that PET and MR images can be acquired simultaneously. Integrated PET/MRI systems use 
detector systems based on either avalanche photodiodes or SiPMs that are insensitive to the magnetic field. The simultaneous measurement ensures a better spatial agreement of PET and MRI data and provides a unique opportunity for 4-dimensional acquisitions, for example, to perform motion correction without the need for respiratory motion sensors.

Disadvantages of PET/MRI include the high costs and the fact that ferromagnetic metallic implants are contraindications for MRI. Moreover, one of the big challenges for PET/MRI is attenuation correction. Accurate attenuation correction is required for quantitative PET/ MRI, which is important for dosimetry in radionuclide therapy. CT images are ideal for attenuation correction as they provide electron density images, whereas MR images give information about proton density, making them less suitable for attenuation correction. Moreover, MR images are often transaxially truncated. Despite the challenges in MRbased attenuation correction, segmentation-, template-, or atlas-based techniques can be used to derive electron density information from MR images [92]. Alternatively, the attenuation maps may be estimated using algorithms that use the time-of-flight emission or transmission data [93].

\section{Other modalities for guidance}

Imaging modalities other than nuclear imaging are also used to guide radionuclide therapy. For example, fluoroscopic imaging is used for radionuclide therapies that require real-time image guidance, such as assisting in the positioning of catheters for liver radioembolization [94]. With the development of interventional MRI scanners, these procedures may also be performed in the future under MRI guidance for increased soft tissue contrast and the absence of radiation dose. Intratumoural injection of radionuclides may also be performed under guidance from interventional CT images or using ultrasound $[95,96]$

\section{Future perspectives}

\section{Simultaneous $x$-ray and nuclear imaging}

To date, no real-time hybrid imaging modalities for interventional purposes have been developed that combine simultaneously acquired nuclear and anatomic images. Fluoroscopic imaging in conjunction with real-time nuclear imaging would provide the physician with valuable information during procedures such as ${ }^{90} \mathrm{Y}$ liver radioembolization by imaging the distribution of the radionuclide in relation to the anatomy and the interventional instruments, thereby improving therapeutic efficiency. Imaging of the same field of view can be achieved by placing an $\mathrm{x}$-ray tube, an $\mathrm{x}$-ray detector, and a $\mathrm{y}$-camera in one line [32]. Since straightforward combination of these elements would block the line of views, a $\gamma$-camera geometry that looks around the $x$-ray tube was developed. A prototype was built using a mobile $\mathrm{C}$-arm and a $\gamma$-camera with a 4-pinhole collimator.
Measurements with the hybrid imaging prototype that combines simultaneous $x$-ray and nuclear imaging of the same field of view have demonstrated the feasibility of real-time simultaneous hybrid imaging in the intervention room (Figure 2.5).

A
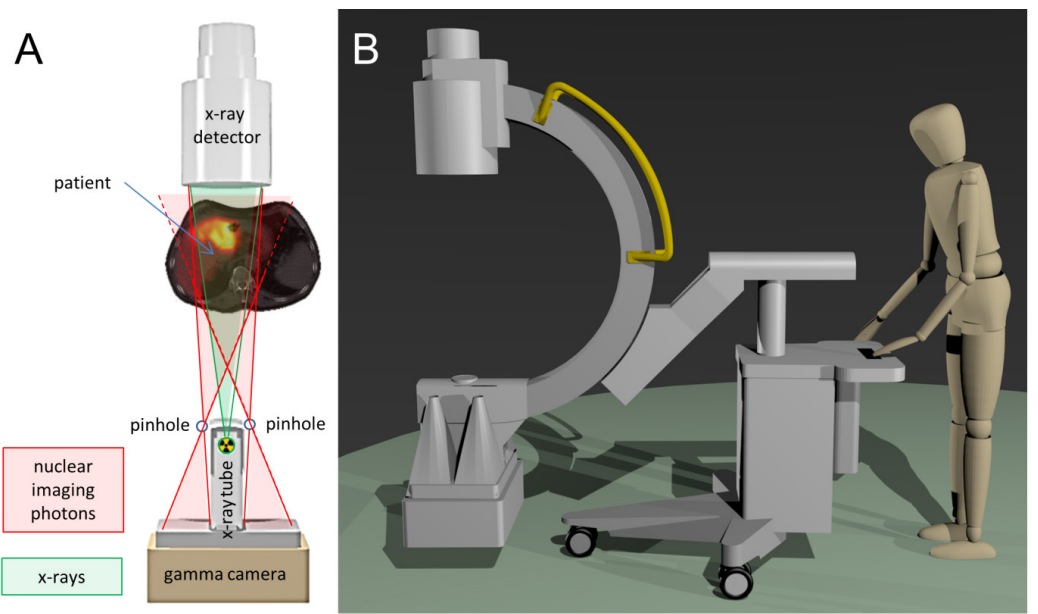

Figure 2.5: (A) Hybrid $\mathrm{c}$-arm showing the field of views of the pinhole collimator and the $x$-ray detector. (B) Entire system [32].

\section{PET versus SPECT}

Generally, SPECT is less expensive than PET in the sense of initial investment and isotopes. However, PET outperforms SPECT with respect to sensitivity, resolution, and quantitative capabilities. One approach to improving the quality of nuclear images is therefore to replace SPECT tracers with positron-emitting PET tracers. Examples include the use of diagnostic ${ }^{124}$-metaiodobenzylguanidine (MIBG) PET instead of ${ }^{123}$-MIBG SPECT before ${ }^{131}$ I-MIBG therapy (Figure 2.6) or the use of low-abundance internal pair production of ${ }^{90} \mathrm{Y}$ for ${ }^{90} \mathrm{Y}$ PET instead of Bremsstrahlung ${ }^{90} \mathrm{Y} \mathrm{SPECT}$ for dosimetry in ${ }^{90} \mathrm{Y}$ liver radioembolization (Figure 2.7) [97-99]. 

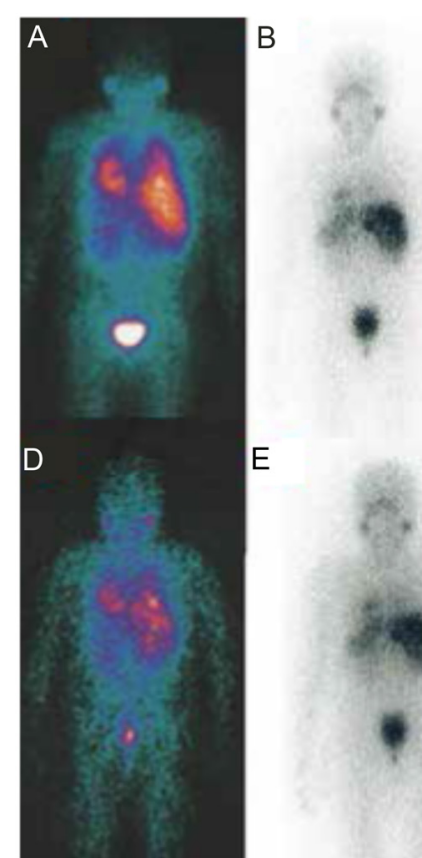

Figure 2.6: Diagnostic 123I-MIBG planar acquisitions at $4 \mathrm{~h}(\mathrm{~A})$ and $24 \mathrm{~h}$ (D) after injection, post-therapy 1311-MIBG planar acquisitions at $24 \mathrm{~h}(\mathrm{~B})$ and $48 \mathrm{~h}$ (E) after injection, and 124-MIBG maximum-ntenst projection acquisitions at $24 \mathrm{~h}(\mathrm{C})$ and $48 \mathrm{~h}$ (F) after injection [97]. Resolution advantage of $124 \mathrm{PET}$ over 1231 and 131 SPECT is clear.

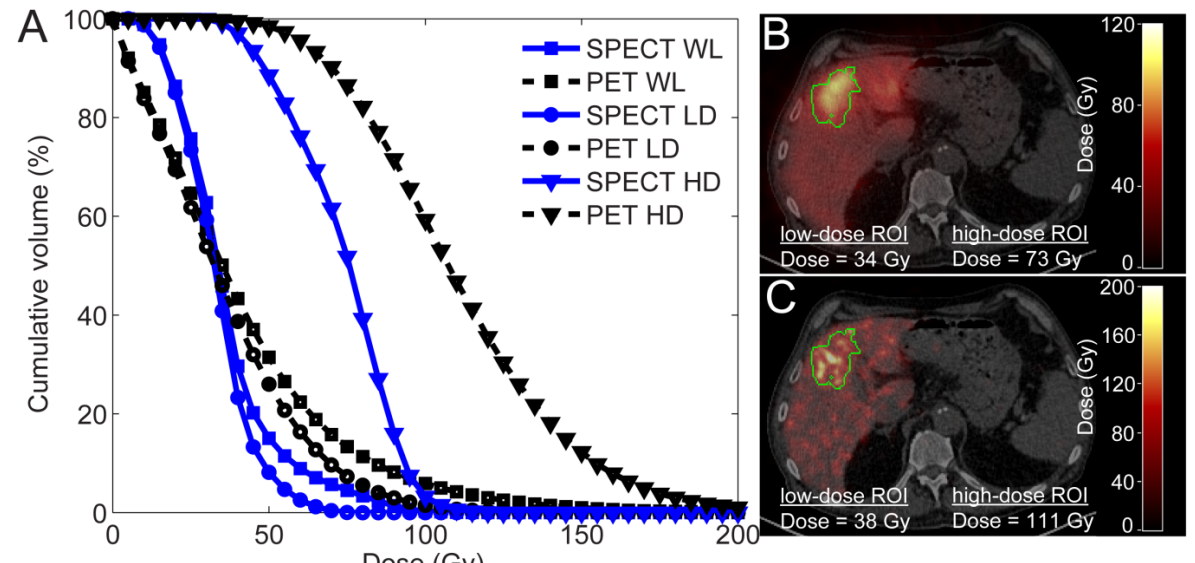

Dose (Gy)

Figure 2.7: Comparison of dose estimates based on ${ }^{\circ 0} \mathrm{Y}$ PET and ${ }^{\circ 0} \mathrm{Y}$ SPECT images after radioembolization procedure. (A) Graph shows cumulative dose-volume histogram of whole liver (WL), low-dose region of interest (LD), and high-dose region of interest (HD). Same transversal slice through SPECT-based dose map fused with CT (B) and through PET-based dose map (C) shows advantage of PET over SPECT in terms of resolution and effect on calculated dose distribution [100].
These advances spur the question of whether there is a future for SPECT systems in the nuclear medicine department. Some have argued that PET may completely replace scintigraphy and SPECT in the future [101]. However, the type of decay determines whether SPECT or PET is used for imaging. Finding suitable and cost-effective positron-emitting alternatives with a half-life long enough for dosimetry remains a challenge for many SPECT radiopharmaceuticals. Moreover, both PET imaging and SPECT imaging are often applied for theranostics, using matched pairs of isotopes to exploit the characteristics of the PET isotopes that are most suitable for imaging ( ${ }^{124}$ land ${ }^{86} \mathrm{Y}$ ) and their $\beta$-emitting counterparts ( ${ }^{1{ }^{11} I}$ and ${ }^{90} \mathrm{Y}$ ) for therapeutic purposes [87]. This is referred to as matched-pairs dosimetry. For example, ${ }^{124}$ is used for pre-therapeutic imaging of suspected recurrence of differentiated thyroid carcinoma, because a low activity of ${ }^{124}$ ( $~ 1 \%$ of therapeutic ${ }^{131}$ | activity) is often sufficient to achieve similar image quality on pre-therapeutic ${ }^{124}$ PET/ CT and post-therapeutic ${ }^{131} \mid$ SPECT/CT [102]. Another example is the use of ${ }^{86} \mathrm{Y} \mathrm{PET}$ before ${ }^{90} Y$ therapy of somatostatin receptor-positive tumours, which has been shown to improve image quality as compared with ${ }^{11}$ In SPECT $[103,104]$. With developments such as matched-pairs dosimetry, SPECT and PET are likely to be used for many years to come because of the complementary capabilities of PET and SPECT radionuclides.

\section{Conclusion}

As the quality of the images used for guidance of radionuclide therapy are continuously improved, the outcome and efficiency of radionuclide therapies may also be improved. For example, improved pre-therapeutic image quality may lead to improved patient selection and, subsequently, to increased accuracy of required dose estimates, paving the way for dosimetry-guided individualized doses. In turn, this development would have the potential to reduce the occurrence of side effects and increase the probability of a successful ablation of the tumour. Technical advances that benefit imaging in radionuclide therapy include developments in algorithms and instrumentation for traditional nuclear imaging modalities, as well as the development of novel modalities. 


\section{Abstract}

Purpose: To develop and evaluate a dual layer detector capable of acquiring intrinsically registered real-time fluoroscopic and nuclear images in the intervention room.

Methods: The dual layer detector consists of an $\mathrm{x}$-ray flat panel detector placed in front of a gamma camera with cone beam collimator focused at the $x$-ray focal spot. This design relies on the x-ray detector absorbing the majority of the $x$-rays, while being more transparent to the higher energy gamma photons. A prototype was built and dynamic phantom images were acquired. In addition, spatial resolution and system sensitivity (evaluated as counts detected within the energy window per second per $\mathrm{MBq}$ ) were measured with the prototype. Monte Carlo simulations for an improved system with varying flat panel compositions were performed to assess potential spatial resolution and system sensitivity.

Results: Experiments with the dual layer detector prototype showed that spatial resolution of the nuclear images was unaffected by the addition of the flat panel (full width at half maximum: $13.6 \mathrm{~mm}$ at $15 \mathrm{~cm}$ from the collimator surface). However, addition of the flat panel lowered system sensitivity by $45 \%$ to $60 \%$, owing to the non-optimized transmission of the flat panel. Simulations showed that an attenuation of $27 \%$ to $35 \%$ of the gamma rays in the flat panel could be achieved by decreasing the crystal thickness and housing attenuation of the flat panel.

Conclusions: A dual layer detector was capable of acquiring real-time intrinsically registered hybrid images, which could aid interventional procedures involving radionuclides.

\section{Introduction}

Hybrid imaging modalities are of great importance for oncological imaging because they combine anatomical and nuclear information. However, the use of hybrid imaging modalities (e.g. single photon emission computed tomography/computed tomography (SPECT/CT) in the interventional radiology suite is limited due to the bulky design of these modalities [105]. Interventional nuclear imaging is performed with gamma probes or hand-held gamma cameras, providing real-time feedback about the activity distribution. Interpretation of this information can be difficult for lack of co-registered anatomical information. Therefore, we aim at developing a real-time, simultaneous fluoroscopic and nuclear imaging device, consisting of a c-arm with nuclear imaging capabilities.

In the design of Beijst et al. [32,106], four gamma cameras with pinhole collimators were positioned at the side of thex-ray tube. Although the prototype showed that interventional hybrid imaging was feasible, this design requires an intermediate reconstruction step and additional weight added to the already heavy $x$-ray tube. Also, it has a lower spatial resolution of the nuclear image close to the $x$-ray detector. Instead, we propose a dual layer detector that does not require an intermediate reconstruction step, has better nuclear image resolution and has better weight balance between $\mathrm{x}$-ray source and detector.

The hypothesis of this study was that a dual layer detector is capable of acquiring intrinsically registered real-time fluoroscopic and nuclear images in the intervention room, which may guide interventional procedures involving radionuclides such as radioembolization.

\section{Materials and Methods}

Philips Healthcare supported this research by providing an adapted $x$-ray flat panel detector. The authors had full control over the data and the information submitted for publication.

\section{Geometry}

The dual layer detector consists of a gamma camera with cone beam collimator placed behind a dynamic $x$-ray flat panel (Figure 3.1). The cone beam collimator is focused on the focal spot of the $x$-ray tube, resulting in intrinsically registered $x$-ray and nuclear images (Figure 3.1). The $x$-ray flat panel absorbs the majority of $x$-rays (30-120 keV) but is more transparent to higher energy (140 keV) gamma rays, allowing simultaneous detection. 

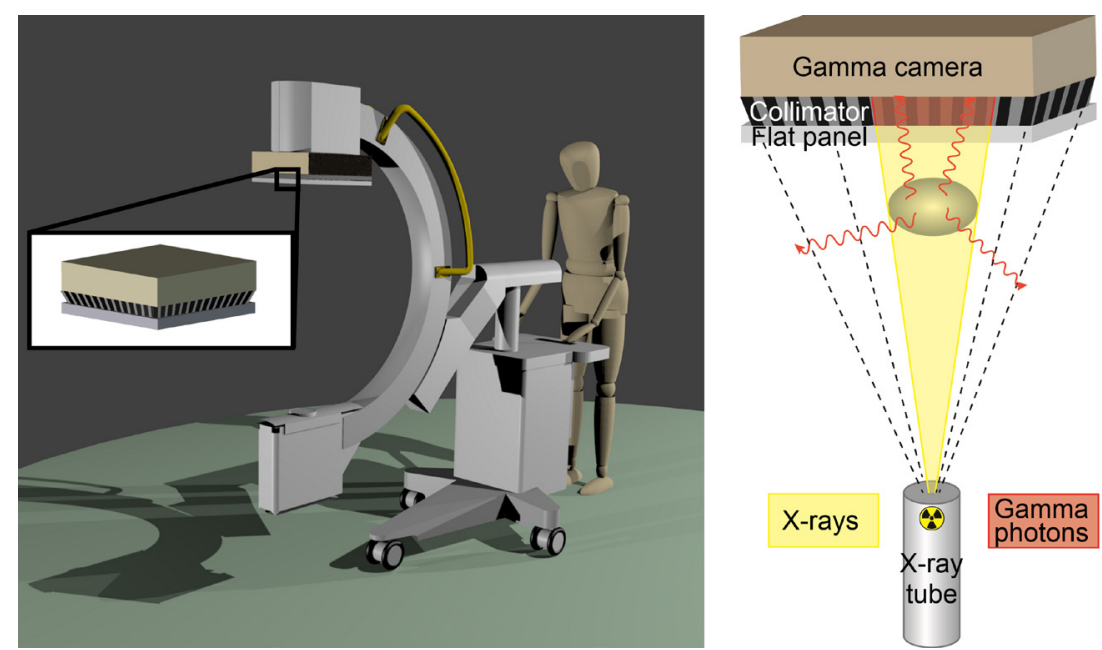

Figure 3.1: Rendering of a mobile $c$-arm with the proposed dual layer detector, consisting of a gamma camera, cone beam collimator and $x$-ray flat panel (left); and schematic overview of the principle of simultaneous detection of nuclear and $x$-ray images (right).

We built (W.J.C.K. and J.H.L.S.) an experimental prototype of the dual layer detector (Figure 3.2). The gamma imaging part of the prototype consisted of a Diacam gamma camera (Siemens Healthcare, Erlangen, Germany) mounted with a low energy cone beam collimator (septal thickness: 0.25 mm; hole diameter: $1.90 \mathrm{~mm}$; hole length: $40 \mathrm{~mm}$; focal distance: $98 \mathrm{~cm}$ ) (Nuclear Fields, Vortum-Mullem, the Netherlands) [107]. The gamma camera had a $9.5 \mathrm{~mm}$ thallium-doped sodium iodide $(\mathrm{Nal}(\mathrm{T}))$ scintillation crystal and a field-of-view (FOV) of $53.3 \times 38.7 \mathrm{~cm}$. The intrinsic resolution of the gamma camera was $10 \mathrm{~mm}$ full width at half maximum (FWHM) as measured with a collimated point source without collimator.

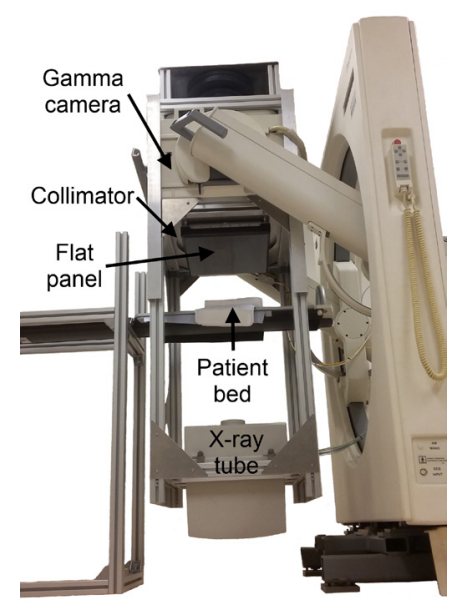

The $x$-ray imaging part consisted of an $\mathrm{x}$-ray tube of a Veradius c-arm (Philips Healthcare, Best, the Netherlands) and a commercially available Pixium ${ }^{\oplus}$ 3040 flat panel (Trixell, Moirans, France) [108] The $x$-ray flat panel had a $750 \mu \mathrm{m}$ thallium-doped cesium iodide (CsI(TI)) detection layer, $154 \mu \mathrm{m}$ pixel pitch, a FOV of $29.6 \times 38.2 \mathrm{~cm}$ and a total thickness of $7.5 \mathrm{~cm}$. The flat panel was first modified by Philips Healthcare to improve the transmission of gamma

Figure 3.2: Prototype setup, consisting of a gamma camera with cone beam collimator (focus at $98 \mathrm{~cm}$ ), modified flat panel and $x$-ray tube. rays over the original detector by removing the lead shielding and some excess aluminum at the backside of the assembly.

\section{Phantom experiments}

To illustrate potential applications of the dual layer detector, two phantom experiments were performed (S.V. and B.K.) where dynamic nuclear and fluoroscopic images were acquired simultaneously. In the first experiment, an $8.4 \mathrm{MBq}$ cobalt-57 $\left({ }^{57} \mathrm{Co}\right)$ pen marker was moved within the thorax of the RS-800 phantom (Radiological Support Devices, Long Beach, CA). The thorax was filled with air and the heart, liver and lungs were removed while all bone structures of the thorax were present.

In the second experiment, a liver-shaped phantom containing a $40 \mathrm{~mm}$ diameter sphere representing a liver tumour was positioned on a translating stage to simulate breathing motion (amplitude: $2 \mathrm{~cm}$, period: $5 \mathrm{~s}$ ). The sphere was filled with $16 \mathrm{MBq}$ of

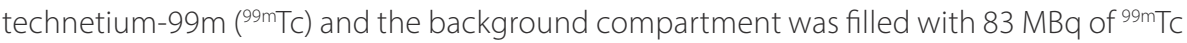
(concentration ratio 1:10), representing a ${ }^{99 m} \mathrm{mc}$ macroaggregated albumin ( ${ }^{99 \mathrm{mTC}} \mathrm{T}-\mathrm{MAA}$ ) procedure preceding yttrium-90 radioembolization [109].

For both phantom experiments, dynamic nuclear data was acquired in list-mode with a $15 \%$ wide energy window centered at the photopeak. Every $250 \mathrm{~ms}$, list-mode data of the prior 500 ms was binned into image frames visualised at a frame rate of $4 \mathrm{~Hz}$. List mode data acquired during and shortly after the x-ray pulses was left out (21 ms per x-ray pulse) (Figure 3.3). Nuclear images ( $2 \times 2 \mathrm{~mm}$ pixel size) were corrected for non-uniformities with a flood map and post-filtered with a $24 \mathrm{~mm}$ FWHM Gaussian filter. Fluoroscopic images were acquired at $51 \mathrm{kVp}$ and $0.515 \mathrm{~mA}$ (first phantom experiment) and at $52 \mathrm{kVp}$ and 0.555 $\mathrm{mA}$ (second phantom experiment) with automatic exposure control, both at a frame rate of $4 \mathrm{~Hz}$, in alignment with the $4 \mathrm{~Hz}$ nuclear images. Registration of the fluoroscopic image with the nuclear image was achieved by applying a rigid transformation (rotation, translation, magnification) calculated from three ${ }^{57} \mathrm{Co}$ pen marker measurements.

\section{Nuclear image quality}

To assess nuclear image quality, static measurements were performed (S.V. and B.K.) using

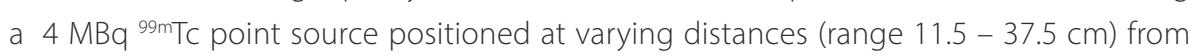
the collimator surface with $60 \mathrm{~s}$ acquisition time with and without flat panel in place, to study the influence of the flat panel on nuclear image spatial resolution and system sensitivity. Counts were acquired in a 15\% energy window centered at $140 \mathrm{keV}$. Pixel size was $2 \times 2 \mathrm{~mm}$. Spatial resolution was defined as the FWHM of the point spread function. System sensitivity was expressed in counts per second (CPS)/MBq being detected within 
the energy window. In addition, system sensitivity was measured using a $200 \mathrm{MBq}{ }^{99 m} \mathrm{TC}$ flood source of $53 \times 40 \mathrm{~cm}$.

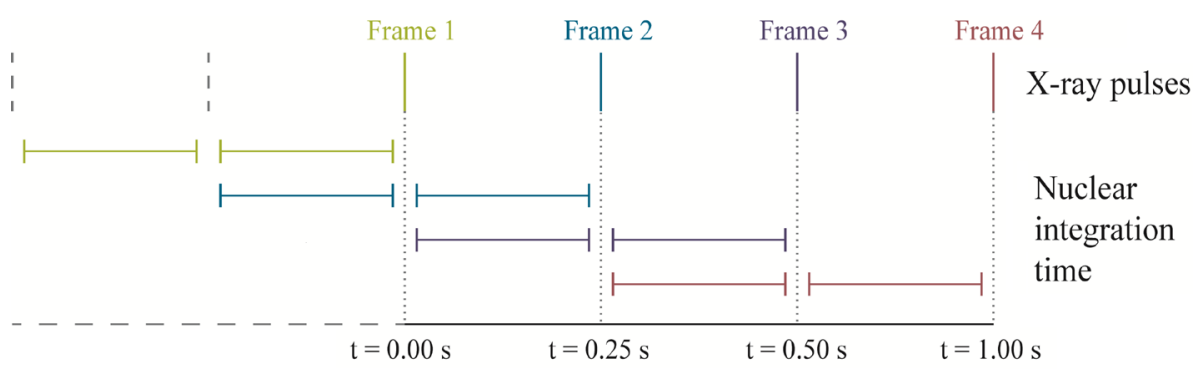

Figure 3.3: Schematic overview of hybrid data acquisition scheme. Every $250 \mathrm{~ms}$, list-mode data of the prior $500 \mathrm{~ms}$ was binned into image frames which were visualised at a frame rate of $4 \mathrm{~Hz}$. List-mode data acquired during and shortly after the $x$-ray pulses was left out ( $21 \mathrm{~ms}$ per $\mathrm{x}$-ray pulse).

\section{Simulations}

First, the prototype system was simulated using MCNP6 1.0 (S.V. and B.K.) (12). Since the exact composition of the flat panel was confidential and therefore not disclosed by the manufacturer, the flat panel was modelled as $750 \mu \mathrm{m} \mathrm{Csl,} 700 \mu \mathrm{m} \mathrm{Si}$ (read-out layer) and 26 $\mathrm{mm} \mathrm{Al}$ to match the system sensitivity of the prototype system. The total thickness of the modelled flat panel was identical to the prototype flat panel $7.5 \mathrm{~cm}$, with the remaining $47.5 \mathrm{~mm}$ modelled as air)

Based on this model, improved systems were simulated by (i) reducing the amount of Al to $7 \mathrm{~mm}$, (ii) reducing the flat panel thickness to $1 \mathrm{~cm}$ (thin flat panel), (iii) varying the Csl thickness (range $0.3-0.75 \mathrm{~mm}$ ) and (iv) simulating an intrinsic spatial resolution of the gamma camera of $3 \mathrm{~mm}$ FWHM.

For all combinations of source-collimator distance and flat panel composition, a separate simulation was run with 100 million photons, representing a point source of $1.7 \mathrm{MBq}$ ${ }^{99 m} \mathrm{~m} \mathrm{c}$ with an acquisition time of 60 seconds. The point source was positioned at varying distances from the collimator surface (range $5-31 \mathrm{~cm}$ ). The energy resolution was simulated as 10\%. The intrinsic resolution of the setup with thin flat panels was simulated as $3 \mathrm{~mm}$ FWHM and the intrinsic resolution of the prototype setup (thick flat panel) was simulated as $10 \mathrm{~mm}$ FWHM. Pixel size was $2 \times 2 \mathrm{~mm}$.

\section{Results}

\section{Phantom experiments}

Figure 3.4 and 3.5 show single frame images of the real-time, simultaneously acquired hybrid video of both phantoms (supplemental material 1 and 2), with the x-ray image in grayscale and the nuclear image in colour overlay. Visual inspection of the images showed that good spatial overlap was obtained and no artifacts were visible in either the $x$-ray or nuclear images (agreement by all authors, including a nuclear radiologist with $>20$ year's experience). This shows that the presence of the radioactive tracer had no influence on the quality of the x-ray image. In addition, nuclear images could be acquired with short frame durations, despite the attenuation and scatter of gamma rays by the flat panel.

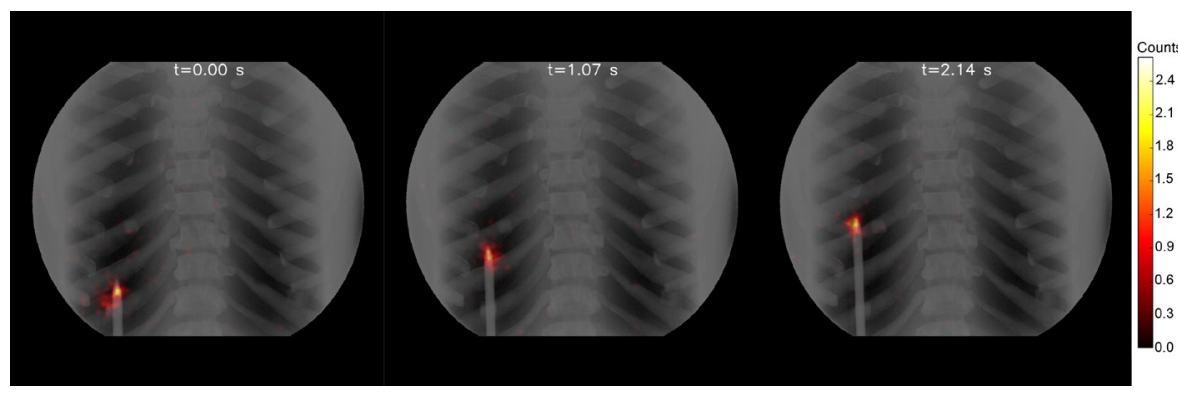

Figure 3.4: Single frame images $(\Delta t=1.07 \mathrm{~s}$ ) of the video of a cobalt-57 pen marker moving inside the thorax of the RS-800 phantom (supplemental material 1). X-ray images are shown in grayscale, nuclear images are shown in colour overlay, scaled between zero and the maximum number of counts.

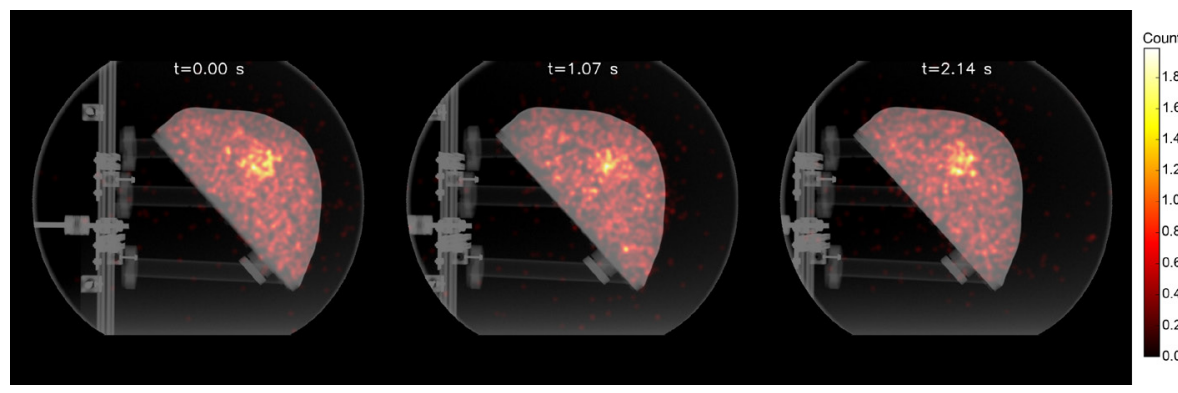

Figure 3.5: Single frame images $(\Delta t=1.07 \mathrm{~s})$ of the video of the moving liver-shaped phantom acquired with the prototype setup (supplemental material 2). X-ray images are shown in grayscale, nuclear images are shown in colour overlay, scaled between zero and the maximum number of counts. The white arrow indicates the position of the hot sphere in the first frame $(t=0.00 \mathrm{~s})$. 


\section{Nuclear image quality}

Figure $3.6 \mathrm{a}$ and b show the measured system sensitivity and spatial resolution of the prototype, with and without flat panel, and the simulations of the prototype system with flat panel. The simulations were in agreement with the measurements of the prototype (difference in system sensitivity and spatial resolution less than 14\% and 9\%, respectively). For the point source, measured at the center of the camera, incorporating the flat panel into the setup reduced the system sensitivity by $60 \%$. Reduction was $45 \%$ when measured with the flood source. The difference in measured system sensitivity represents the inhomogeneity of the flat panel. Spatial resolution was unaffected by addition of the flat panel (difference less than 7\%).

$A_{250}$
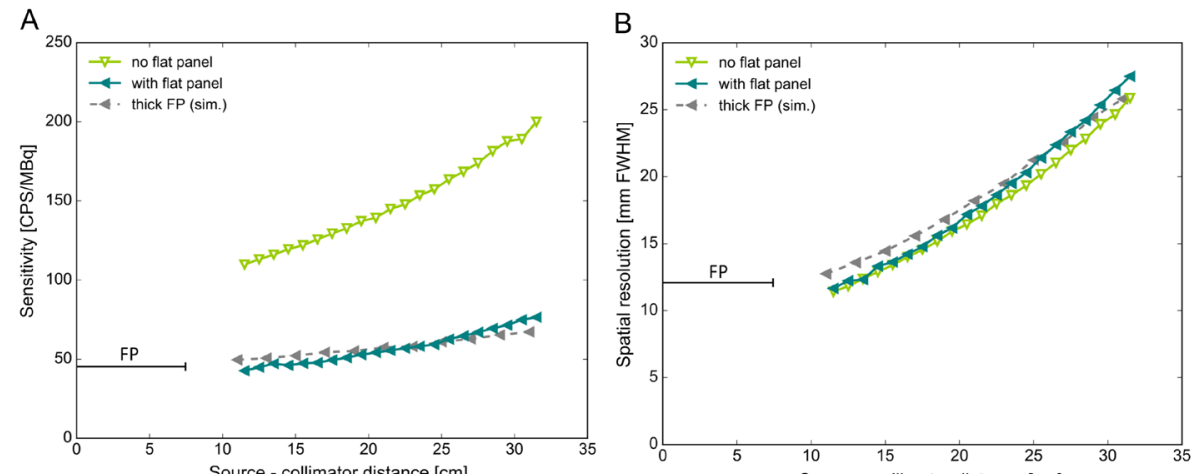

C
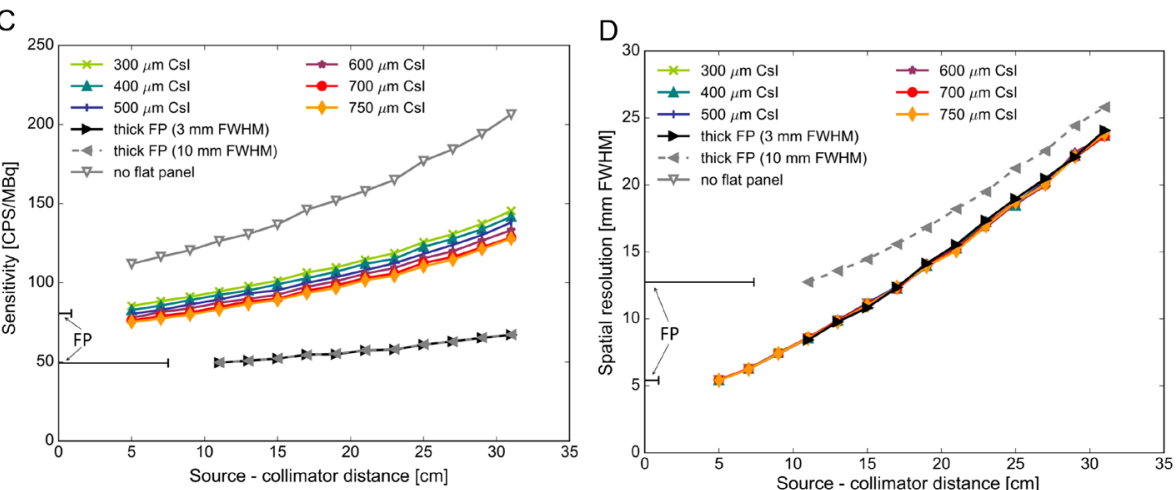

Figure 3.6: Measured and simulated system sensitivity (a) and spatial resolution (b) of the prototype system The simulated thick flat panel was modelled to match these measurements. Simulated system sensitivity (c) and simulated spatial resolution (d) for the current thick flat panel and the modelled thin flat panels. In all figures, the thickness of the flat panel is indicated by the horizontal black line. CPS = counts per second, Csl $=$ cesium iodide, $\mathrm{FP}=$ flat panel, $\mathrm{FWHM}=$ full width at half maximum)
Figure $3.6 \mathrm{c}$ and $\mathrm{d}$ show the simulated system sensitivity and spatial resolution of the improved systems. Incorporating a thin flat panel into the setup reduced the system sensitivity by $27 \%$ - 35\%, depending on the CsI crystal thickness. Improving the intrinsic resolution of the gamma camera did not influence the system sensitivity. Spatial resolution was unaffected (difference less than 2\%) by reducing the flat panel thickness or varying Csl thickness. However, a thinner flat panel allowed smaller source-collimator distances, which improved the spatial resolution. Improving the intrinsic resolution of the gamma camera also improved the spatial resolution of the system.

\section{Discussion}

Interventional procedures could benefit from real-time intrinsically registered hybrid images for improved localization and treatment guidance. Simulations and measurements with the prototype system demonstrated that a dual layer detector, consisting of a gamma camera with cone beam collimator and x-ray flat panel, was capable of acquiring such hybrid images. Addition of the flat panel did not affect spatial resolution of the nuclear image, system sensitivity decreased by $45 \%$ to $60 \%$.

Availability of hybrid images would potentially benefit multiple procedures, such as sentinel node procedures, biopsies and radioembolization procedures. For radioembolization procedures, the main advantage is that patients will not have to be transferred to the nuclear medicine department for assessment of the pre-treatment procedure with ${ }^{99 \mathrm{mT} C \text { - }}$ MAA. This would make the procedure more time efficient and would allow for one-day radioembolization procedures $[110,111]$. In a one-day procedure, the vascular sheath is not removed, in contrast to the standard procedure, ensuring equal injection position for ${ }^{99 m} \mathrm{TC}-\mathrm{MAA}$ and microspheres. When nuclear images can be acquired in the intervention room, movement of the vascular sheath is limited because the patient does not have to change beds for assessment of the ${ }^{99 \mathrm{~m} T \mathrm{C}-M A A}$ distribution. This improves the prognostic power of the pre-treatment procedure [112]. However, in principle, any procedure using radionuclides and $\mathrm{x}$-ray imaging could benefit from our dual layer detector.

Transmission of gamma rays through the flat panel can be substantially improved by reducing the amount of aluminum surrounding the flat panel. In addition, repositioning the read-out electronics would improve homogeneity of the gamma ray transmission. The position of some large electronic components explains the difference in system sensitivity when measured with a point source compared to a flood source $(60 \%$ and $45 \%$ reduced system sensitivity, respectively). 
Modifications to our prototype flat panel only affected the housing and therefore did not influence its imaging properties, so from the x-ray point of view every fluoroscopic setting can be used. However, from a nuclear point of view, the current prototype allows the use of 40-80 kVp tube voltages, depending on the tube current. For our phantom experiments, $x$-ray images were acquired at $\sim 50 \mathrm{kVp}$, since the phantoms were small and did not require high tube voltages. In clinical practice, higher tube voltages are used. Although higher tube voltages cause more photons to penetrate the $x$-ray detector and create interactions in the gamma camera, we believe that this effect can be mitigated by modification of the PMT circuitry, and/or the addition of a high pass filter, and/or by performing interleaved measurements (required when tube voltage approaches photopeak energy) [113]. Such interleaved measurements were used for the acquisition of the phantom images and assumed in our simulations, obviating the need for simulation of the x-ray pulses.

A limitation of our proposed detector is the reduced system sensitivity of the gamma camera due to the attenuation in the flat panel and the short frame duration required for real-time imaging. Although these factors will negatively influence nuclear image quality in terms of contrast and noise, the aim of our system is not to provide the physician with images of the highest diagnostic quality. In interventional procedures, the diminished nuclear image quality may be compensated by providing additional information that is otherwise not available. Also, image quality may be improved by image processing, exploiting the dynamic nature of the image data by frame averaging, as is commonly done in fluoroscopy.

In conclusion, we have demonstrated the feasibility of acquiring real-time and intrinsically registered fluoroscopic and nuclear images of the same FOV, by means of a dual layer detector. Such hybrid images may be used advantageously in interventional procedures involving radionuclides, such as radioembolization. In a more mature setup, we plan to redesign the aluminum housing of the flat panel and reposition the read-out electronics. This will result in the transmission of more gamma photons through the flat panel. The final design of our setup will be a trade-off between nuclear and fluoroscopic image quality. With regard to flat panel composition, a thicker Csl layer would lead to both better fluoroscopic dose efficiency and a lower sensitivity for gamma photons. The required Csl thickness will be further investigated in future research. 
Comparison of three different therapeutic particles in radioembolization of locally advanced intrahepatic cholangiocarcinoma

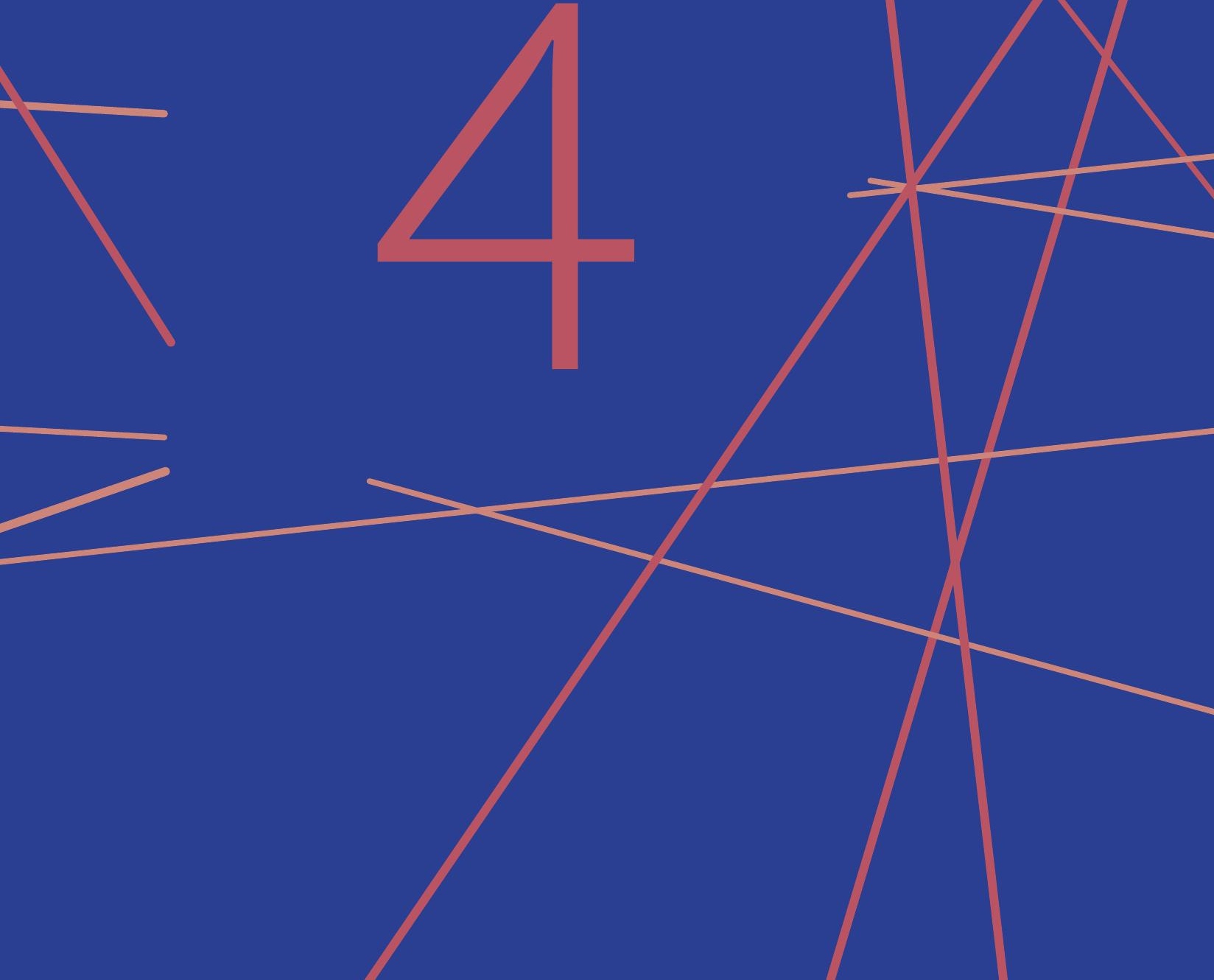




\section{Abstract}

Purpose: To compare three different therapeutic particles used for radioembolization in advanced intrahepatic cholangiocarcinoma.

Methods: Yttrium-90 ( $\left.{ }^{90} \mathrm{Y}\right)$ glass, ${ }^{90} \mathrm{Y}$ resin, and holmium-166 (166 $\left.\mathrm{Ho}\right)$ microsphere prescribed activity was calculated as per manufacturer's recommendation. Post-treatment quantitative ${ }^{90} \mathrm{Y} \mathrm{PET/CT}$ and quantitative ${ }^{166} \mathrm{HO}$ SPECT/CT was used to determine tumour absorbed dose, whole healthy liver absorbed dose, treated healthy liver absorbed dose, tumour to non-tumour ratio (T/N), lung absorbed dose, and lung shunt fraction. Response was assessed using RECIST 1.1 criteria and PET-based change in tumour lesion glycolysis (TLG). Toxicity was assessed using the hepatotoxicity or radioembolization-induced liver disease (REILD) classification.

Results: Six ${ }^{90} \mathrm{Y}$ glass, eight ${ }^{90} \mathrm{Y}$ resin, and seven ${ }^{166} \mathrm{Ho}$ patients were included for analysis. Mean administered activity was $2.6 \mathrm{GBq}$ for ${ }^{90} \mathrm{Y}$ glass, $1.5 \mathrm{GBq}$ for ${ }^{90} \mathrm{Y}$ resin, and $7.0 \mathrm{GBq}$ for ${ }^{166} \mathrm{Ho}$. Tumour and treated healthy liver absorbed dose were significantly higher for ${ }^{90} \mathrm{Y}$ glass than for ${ }^{90} \mathrm{Y}$ resin and ${ }^{166 \mathrm{Ho}}$ microspheres (mean tumour absorbed dose: $197 \mathrm{~Gy}{ }^{90} \mathrm{Y}$ glass vs. $73 \mathrm{~Gy}{ }^{90} \mathrm{Y}$ resin and $50 \mathrm{~Gy}{ }^{166} \mathrm{Ho}$, mean treated healthy liver absorbed dose: $79 \mathrm{~Gy}$ ${ }^{90} \mathrm{Y}$ glass vs. $37 \mathrm{~Gy}{ }^{90} \mathrm{Y}$ resin and $31 \mathrm{~Gy}{ }^{166} \mathrm{Ho}$ ). The whole healthy liver absorbed dose and $\mathrm{T} / \mathrm{N}$ did not significantly differ between the particles. The three groups showed similar toxicity and response.

Conclusion: The therapeutic particles used for radioembolization differ from each other, and showed significant differences in absorbed dose, although toxicity and response were similar for all groups. This emphasises the need for separate dose constraints and dose targets for each particle.

\section{Introduction}

Intrahepatic cholangiocarcinoma (ICC) is the second most common malignancy arising from the liver [114]. Surgical resection (or liver transplantation) is the only curative option. However, at the time of diagnosis, surgical resection is no longer an option for $68 \%$ of patients, 70\% due to unresectable disease and 30\% due to metastatic disease [115].

An alternative liver-directed treatment for locally advanced ICC is radioembolization, which has shown promising results in several clinical studies [116-121]. During radioembolization treatment, microspheres are injected in (a branch of) the hepatic artery and will preferentially lodge in and around the tumour(s) where they will locally irradiate the tumour(s). Currently, three types of microspheres are commercially available: yttrium-90 ( $\left.{ }^{90} \mathrm{Y}\right)$ glass microspheres (TheraSpheres, Boston Scientific), ${ }^{90} \mathrm{Y}$ resin microspheres (SIR-spheres, Sirtex Medical), and holmium-166 ('166 Ho) poly(L-lactic acid) microspheres (QuiremSpheres, Quirem Medical B.V.). The physical characteristics of these microspheres are listed in table 4.1.

Table 4.1: Microsphere physical characteristics [22].

\begin{tabular}{lccc}
\hline & ${ }^{90} \mathbf{Y}$ glass & ${ }^{90} \mathbf{Y}$ resin & ${ }^{{ }^{166}} \mathbf{H o}$ poly(L-lactic acid) \\
\hline Diameter: mean $(\mu \mathrm{m})$ & 25 & 32 & 30 \\
Diameter: range $(\mu \mathrm{m})$ & $20-30$ & $20-60$ & $15-60$ \\
Specific activity $(\mathrm{Bq} / \mathrm{sph}$-re) & $150-2500$ & 50 & $240-375$ \\
Emitted energy per GBq $(\mathrm{J} / \mathrm{GBq})$ & 50 & 50 & 15.9 \\
Range beta's in tissue: mean $(\mathrm{mm})$ & 2.5 & 2.5 & 2.5 \\
Range beta's tissue: maximum $(\mathrm{mm})$ & 11 & 11 & 8.7 \\
Half-life (hours) & 64.1 & 64.1 & 26.8 \\
\hline
\end{tabular}

Besides differences in physical characteristics, different methods for calculating the prescribed activity are advised. For ${ }^{90} \mathrm{Y}$ glass and ${ }^{166} \mathrm{Ho}$ microspheres, a mono-compartment model is most frequently used, where the aim is to reach a desired average absorbed dose in a target volume (either the whole liver, or specific segments) assuming a completely homogeneous activity distribution within this target volume. This desired absorbed dose is specified as $60 \mathrm{~Gy}$ for ${ }^{166} \mathrm{Ho}$ microspheres, and 80-150 Gy for ${ }^{90} \mathrm{Y}$ glass microspheres [26-28]. For ${ }^{90} Y$ resin microspheres, the body surface area (BSA) is most commonly used, in which the prescribed activity is based on the body surface area and the percentage of tumour involvement. Downside of these methods is that the actual dose distribution is heterogeneous, which is not taken into account. The so-called partition model is therefore increasingly used. This more advanced treatment planning method takes differences in 
activity concentration in different compartments into account, including the tumour to non-tumour concentration ratio (T/N). However, although more advanced, it does not take differences in heterogeneous activity distribution within the specified compartments into account [122].

Dose-response and dose-toxicity studies are important to set targets for tumour absorbed dose and limits for healthy liver absorbed dose, making the treatment more personalised. There is increasing literature on dose-effect relationships, and there is a wide variety on optimal absorbed dose reported [123-126]. These differences are cause by differences in response criteria used, absorbed dose calculations, scan modalities, tumour types, and microsphere types [122].

In this study we aim to compare the three particles used for radioembolization, to understand the differences and make knowledge-based decisions in future treatment selection and planning. Using quantitative imaging we aim to compare delivered dose to the tumours, healthy liver parenchyma, and lungs, and in addition try to link these parameters with tumour response and toxicity.

\section{Material and methods}

\section{Treatment procedures and planning}

Patients with locally advanced ICC, adequate clinical condition (Eastern Cooperative Oncology Group performance score $\leq 2$ ) and adequate liver function (Child Pugh score $<B 8$ ), who were treated with radioembolization, and underwent pre-treatment $2-\left[{ }^{18} \mathrm{~F}\right]$ FDG PET/CT, were included in this retrospective study. All patients were considered to be unresectable at multidisciplinary tumour board, and/or failed conventional chemotherapy (gemcitabine + cisplatin) or were considered ineligible for chemotherapy (due to comorbidities)

Standard radioembolization work-up included 2-[18F]FDG PET/CT and multiphasic CT of the liver to establish the extent of the disease and the hepatic vasculature. Candidates were discussed in a multidisciplinary tumour board, and eligible patients underwent pretreatment angiography in which $150 \mathrm{MBq}$ of technetium-99m labelled macroaggregated albumin ([99mTc]TC-MAA) (TechneScan LyoMAA, Mallinckrodt Medical B.V. or Pulmocis, Curium Pharma) was administered, followed by planar scintigraphy and SPECT/CT, to estimate the lung shunt fraction (LSF) and to exclude extrahepatic activity depositions.
Prescribed activity was calculated as per manufacturer's recommendation: BSA-method for ${ }^{90} \mathrm{Y}$ resin microspheres [127], and the medical internal radiation dose (MIRD) monocompartment model for ${ }^{90} \mathrm{Y}$ glass and ${ }^{166} \mathrm{Ho}$ microspheres [24,25]. Chosen target dose for ${ }^{90} \mathrm{Y}$ glass microspheres was based on visual assessment of tumour targeting on $\left.{ }^{99 \mathrm{~m}} \mathrm{TC}\right] \mathrm{C} \mathrm{C}-$ MAA SPECT: 120 Gy for good tumour targeting, 80-100 Gy for moderate to poor tumour targeting, or in rare cases 200 Gy when there was tumour ablative intent (i.e. radiation segmentectomy). The target dose for ${ }^{166} \mathrm{Ho}$ microspheres was $60 \mathrm{~Gy}$ in all cases.

\section{Post-treatment imaging}

Post-treatment quantitative imaging of ${ }^{90} \mathrm{Y}$ glass and ${ }^{90} \mathrm{Y}$ resin microspheres was performed with PET/CT (Siemens Biograph $\mathrm{mCT}, 15$ minutes per bed position, 4 iterations with 21 subsets and $5 \mathrm{~mm}$ full width at half maximum Gaussian filter, including time of flight information, resolution recovery, scatter, random, and CT-based attenuation correction, voxel size of $4.1 \times 4.1 \times 3.0 \mathrm{~mm}^{3}$ ) within 24 hours after microsphere administration. Quantitative imaging of ${ }^{166} \mathrm{Ho}$ microspheres was performed with SPECT/CT (Siemens Symbia T16 or T2, energy window of 75-87 keV, 20 iterations with 8 subsets, including CT-based attenuation, resolution recovery, and Monte-Carlo based scatter correction [61] (which is not included in standard clinical reconstructions, but necessary to acquire quantitative images), voxel size of $4.8 \times 4.8 \times 4.8 \mathrm{~mm}^{3}$ ) three to six days after microsphere administration. Three months follow up imaging consisted of multiphasic CT for all patients and for 16 patients also of 2-[18 $\mathrm{F}] \mathrm{FDG}$ PET/CT.

\section{Intrahepatic dosimetry}

In analogy to van den Hoven et al. [124], and derived from PERCIST [128], tumours were segmented on baseline 2-[18F]FDG PET/CT as all voxels with a standard uptake value normalized for lean body mass (SUL) higher than two times the SUL mean in the aortic blood pool, and excluding volumes of less than $5 \mathrm{~cm}^{3}$. For each tumour, metabolic activity was recorded as total lesion glycolysis (TLG), which was calculated by multiplying the

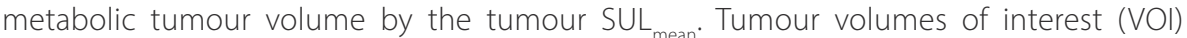
were transferred from 2-[18F]FDG PET/CT to either ${ }^{90} \mathrm{Y} \mathrm{PET/CT} \mathrm{or}{ }^{166} \mathrm{Ho}$ SPECT/CT by rigid registration based on the low dose CTs [129]. In addition, the whole liver and treated liver segments were manually delineated using 3D Slicer (Figure 4.1) [130].

${ }^{90} \mathrm{Y}$ and ${ }^{166} \mathrm{Ho}$ reconstructed activity concentrations were converted into absorbed dose maps by correcting activity for the time of microsphere administration and assuming that all emitted energy was absorbed within the voxel of origin. Mean absorbed dose was recorded for all tumours, whole healthy liver tissue (defined as whole liver VOI minus all tumour VOIs), and treated healthy liver tissue (defined as treated liver VOI minus all tumour VOIs). For tumour and liver tissue a density of $1.04 \mathrm{~g} / \mathrm{mL}$ was assumed [29]. The 
tumour to non-tumour ratio (T/N) was defined as the tumour absorbed dose divided by the treated healthy liver absorbed dose.

\section{Lung dosimetry}

Post-treatment lung shunt absorbed dose (LSD) was determined on ${ }^{90} \mathrm{Y} \mathrm{PET/CT}$ or ${ }^{166} \mathrm{Ho}$ SPECT/CT by segmenting the lungs on the associated CT (using a threshold and excluding the region of the lungs that is $<2 \mathrm{~cm}$ from the liver, Figure 4.1 ) and assuming a lung density of $0.3 \mathrm{~g} / \mathrm{mL}[29,40]$. The post-treatment lung shunt fraction (LSF), defined as the total lung activity divided by the total liver plus lung activity, was determined by extrapolating the activity in the part of the lungs inside the field of view to the lung volume as measured on the pre-treatment 2-[18$\left.{ }^{18}\right]$ FDG-PET/CT and using the activity in the previously delineated liver.

For comparison, estimated LSF and LSD based on pre-treatment planar scintigraphy of ${ }^{99 \mathrm{~m} T c] T c-M A A}$ was calculated. The clinically used estimated LSF was based on the geometric mean of posterior and anterior planar images of [99m Tc]TC-MAA. This estimated LSF was converted to a pre-treatment estimated LSD by multiplying the estimated LSF by the net administered activity (corrected for residual activity measurement) and assuming a lung mass of $1 \mathrm{~kg}$.

\section{Response and toxicity}

Response assessment was performed on a per-lesion basis. Response was defined based on pre- and post-treatment multiphasic CT according to response evaluation criteria in solid tumours, version 1.1 (RESIST 1.1) [131]. In addition, for patients who also underwent follow-up 2-[18F]FDG PET/CT, metabolic response was defined on a per-lesion basis as the percentage change in TLG between pre- and post-treatment 2-[18 $\mathrm{F}]$ FDG PET/CT, in line with PERCIST criteria. Over 45\% reduction in TLG was regarded as partial response, over $75 \%$ increase in $T L G$ was regarded as progressive disease, and anything in between was regarded as stable disease [132].

To assess liver toxicity, post-treatment imaging results (presence of ascites and splenomegaly) and laboratory results were assessed according to the hepatotoxicity or radioembolization-induced liver disease (REILD) classification, as proposed by Braat et al. [133] (Table 4.2).

\section{Statistics}

The different groups of microspheres were compared using the Kruskal-Wallis test. When the Kruskal-Wallis test indicated a significant difference for a metric, the microspheres were tested using the Mann-Whitney $\mathrm{U}$ test to indicate which microsphere(s) were different. A
$5 \%$ significance level was assumed. The relationship between MAA-based lung dosimetry and post-treatment lung dosimetry was tested using linear regression.

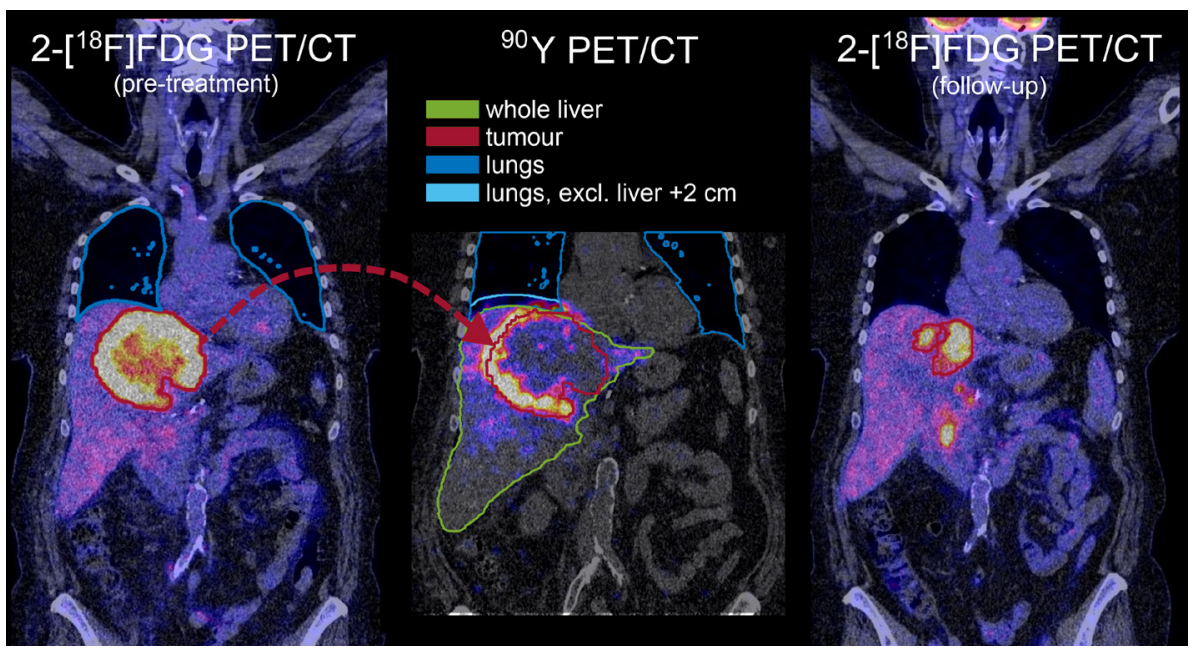

Figure 4.1: Volumes of interest used for analysis. CT in greyscale with PET in colour overlay. The tumour (red) is segmented by thresholding the 2-[18] FED PET (pre-treatment), and this delineation is transferred to the ${ }^{90} \mathrm{Y} \mathrm{PET/CT}$ (or ${ }^{166} \mathrm{Ho}$ SPECT/CT). The lungs (blue) are segmented by thresholding the associated CT of both the 2-[' $\left.{ }^{8} \mathrm{~F}\right] \mathrm{FDG}-\mathrm{PET} / \mathrm{CT}$ (pre-treatment), for lung volume measurement, and the associated CT of the ${ }^{90}$ PET/CT (or ${ }^{166} \mathrm{HO}$ SPECT/CT), for dosimetry where the part of the lungs within $2 \mathrm{~cm}$ of the liver boundary is excluded (light blue). The liver (green) and the treated liver (in this example the same as the liver) are manually delineated on the associated CT of the ${ }^{90}$ PET/CT (or 166 Ho SPECT/CT). The tumour (red) is again segmented by thresholding the 2-['8F]FDG PET (follow-up) for response assessment.

\begin{tabular}{ll} 
Table 4.2: REILD classification, as proposed by Braat et al. [133]. \\
\hline Hepatotoxicity classification & Description \\
\hline Grade 0 & No liver toxicity \\
Grade 1 & Minor liver toxicity, limited to increased aspartate aminotransferase, \\
& alanine aminotransferase, alkaline phosphatase, and/or $\gamma$-glutamyl \\
transpeptidase levels (not exceeding grade 1 CTCAE toxicity) & moderate liver toxicity, with a self-limiting course. No medical \\
Grade 2 & intervention necessary \\
GeILD, managable with noninvasive treatments such as diuretics, & ursodeoxycholic acid, and steroids \\
Grade 4 & $\begin{array}{l}\text { REILD necessitating invasive medical treatment such as paracentesis, } \\
\text { transfusions, hemodialysis, or a TIPS } \\
\text { fatal REILD }\end{array}$ \\
\hline
\end{tabular}
fatal REILD

RELD = radioembolization induced liver disease, $C$ CAE = common terminology criteria for adverse events, TIPS = transjugular intrahepatic portosystemic shun 


\section{Results}

Twenty-three locally advanced ICC patients were treated, of whom seven with ${ }^{90} \mathrm{Y}$ glass microspheres, eight with ${ }^{90} \mathrm{Y}$ resin microspheres, and eight with ${ }^{166} \mathrm{Ho}$ microspheres. Patients were treated between June 2011 and March 2020. One ${ }^{166} \mathrm{Ho}$ microsphere patient was excluded because the activity at the time of SPECT acquisition was considered too high for adequate quantitative imaging (1739 MBq) [16], and one ${ }^{90} \mathrm{Y}$ glass patient was excluded because of a 2-[18 F]FDG negative tumour, precluding PET-based segmentation. Patient characteristics of the remaining 21 patients are summarised in table 4.3. Four ${ }^{90}$ Y glass patients were treated with "first week" microspheres (within seven days postcalibration) and two ${ }^{90} Y$ glass patients were treated with "second week" microspheres.

Table 4.3: Patient characteristics.

\begin{tabular}{|c|c|c|c|}
\hline & ${ }^{90} \mathrm{Y}$ glass & ${ }^{90} \mathrm{Y}$ resin & ${ }^{166} \mathrm{Ho}$ \\
\hline Number of patients & 6 & 8 & 7 \\
\hline Median age in years (range) & $64.5(32-80)$ & $61.5(43-81)$ & $59(45-83)$ \\
\hline Male / female & $2 / 4$ & $3 / 5$ & $2 / 5$ \\
\hline Median liver volume in $\mathrm{mL}$ (range) & $2077(1636-2631)$ & $1842(1231-2182)$ & $2261(1725-4019)$ \\
\hline Number of tumours & 7 & 24 & 11 \\
\hline \multicolumn{4}{|l|}{ Type of radioembolization treatment } \\
\hline Whole liver & 0 & 8 & 4 \\
\hline Lobar & 5 & 0 & 2 \\
\hline Superselective & 1 & 0 & 1 \\
\hline \multicolumn{4}{|l|}{ Previous treatments } \\
\hline Chemotherapy & 2 & 4 & 5 \\
\hline Surgery & 1 & 0 & 0 \\
\hline Chemotherapy + surgery & 1 & 3 & 0 \\
\hline Chemotherapy + radioembolization & 1 & 0 & 0 \\
\hline Surgery + radioembolization & 0 & 0 & 1 \\
\hline none & 1 & 1 & 1 \\
\hline \multicolumn{4}{|l|}{ ECOG performance status } \\
\hline 0 & 5 & 7 & 5 \\
\hline 1 & 1 & 1 & 1 \\
\hline 2 & 0 & 0 & 1 \\
\hline Median administered activity in $\mathrm{MBq}$ (range) & $2273(894-4503)$ & 1541 (729-1943) & $6568(2391-12897$ \\
\hline Median administered energy in GJ (range) & $114(49-225)$ & $77(36-97)$ & $104(38-205)$ \\
\hline $\begin{array}{l}\text { Median activity in } \mathrm{MBq} \text { at time of post- } \\
\text { treatment image acquisition (range) }\end{array}$ & $1818(735-3536)$ & $1397(596-1697)$ & $324(156-374)$ \\
\hline $\begin{array}{l}\text { Median lung shunt fraction based on planar } \\
\text { MAA imaging (range) }\end{array}$ & $3.5 \%(1.9 \%-11.7 \%)$ & $2.2 \%(0.8 \%-14.8 \%)$ & $4.4 \%(1.6 \%-13.4 \%)$ \\
\hline
\end{tabular}

ECOG = eastern cooperative oncology group MAA = macroaggregated albumin
Figure 4.2 shows the T/N, tumour absorbed dose, treated healthy liver absorbed dose, and whole healthy liver absorbed dose for each group of microspheres. The ${ }^{90} \mathrm{Y}$ glass microspheres differ significantly from the ${ }^{90} \mathrm{Y}$ resin and ${ }^{166} \mathrm{Ho}$ microspheres for tumour absorbed dose (mean of 197 Gy vs. 73 Gy and 50 Gy respectively; $p=0.006$ ), and treated healthy liver absorbed dose (mean of 79 Gy vs. 37 Gy and 31 Gy respectively; $p=0.001$ ). The ${ }^{90} \mathrm{Y}$ glass, ${ }^{90} \mathrm{Y}$ resin and ${ }^{166} \mathrm{Ho}$ microspheres did not differ for whole healthy liver absorbed dose (mean of $41 \mathrm{~Gy}, 37 \mathrm{~Gy}$ and $28 \mathrm{~Gy}$ respectively) and tumour to non-tumour ratio $(\mathrm{T} / \mathrm{N})$ (mean of $2.5,2.1$ and 3.0 respectively).
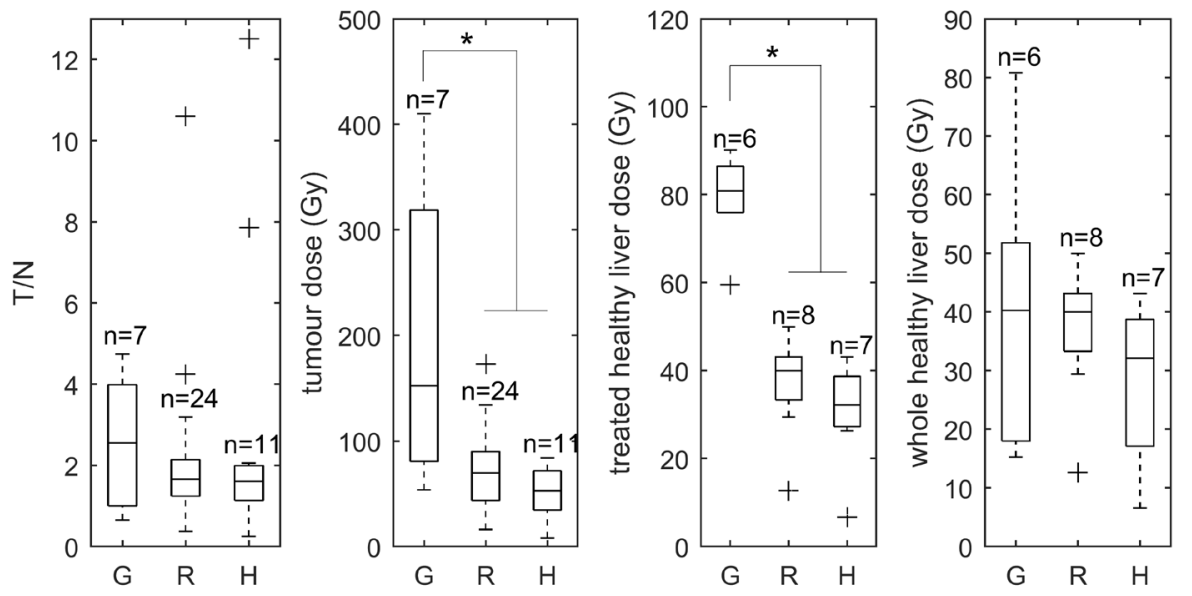

Figure 4.2: Dosimetry of the liver for different microspheres. $G={ }^{90}$ glass microspheres, $R={ }^{90}$ resin microspheres, $\mathrm{H}={ }^{100 \mathrm{H}} \mathrm{m}$ microspheres, $\mathrm{T} / \mathrm{N}=$ tumour to non-tumour activity concentration ratio. * denotes significant difference $(p<0.05)$ between groups. The number above the boxplots for $\mathrm{T} / \mathrm{N}$ and tumour dose indicates the number of tumours. The number above the boxplots for treated healthy liver dose and whole healthy liver dose indicates the number of patients.

The LSD was below 30 Gy and the LSF was below 20\% for all patients (Figure 4.3). Both LSD and LSF did not show significant differences between groups when based on $\left.{ }^{99 \mathrm{~m} T \mathrm{TC}}\right]$ TC-MAA planar scintigraphy, but did show significant differences when based on posttreatment imaging. For the LSD, all three groups of microspheres were significantly different from each other (mean of $5.0 \mathrm{~Gy}$ for ${ }^{90} \mathrm{Y}$ glass vs. $1.3 \mathrm{~Gy}$ for ${ }^{90} \mathrm{Y}$ resin vs. $0.1 \mathrm{~Gy}$ for ${ }^{166} \mathrm{Ho} ; \mathrm{p}=0.001$ ). For the LSF the ${ }^{166} \mathrm{Ho}$ microspheres differ significantly from the ${ }^{90} \mathrm{Y}$ glass and ${ }^{90} \mathrm{Y}$ resin microspheres (mean of $0.2 \%$ for ${ }^{166} \mathrm{Ho}$ vs. $3.7 \%$ for ${ }^{90} \mathrm{Y}$ glass and $1.5 \%$ for ${ }^{90} \mathrm{Y}$ resin; $\mathrm{p}=0.002$ ). None of the particles showed a linear trend with the MAA particles, neither for LSD nor LSF (all 95\% confidence intervals for slope include 0 and all $R^{2}<0.5$ ). 

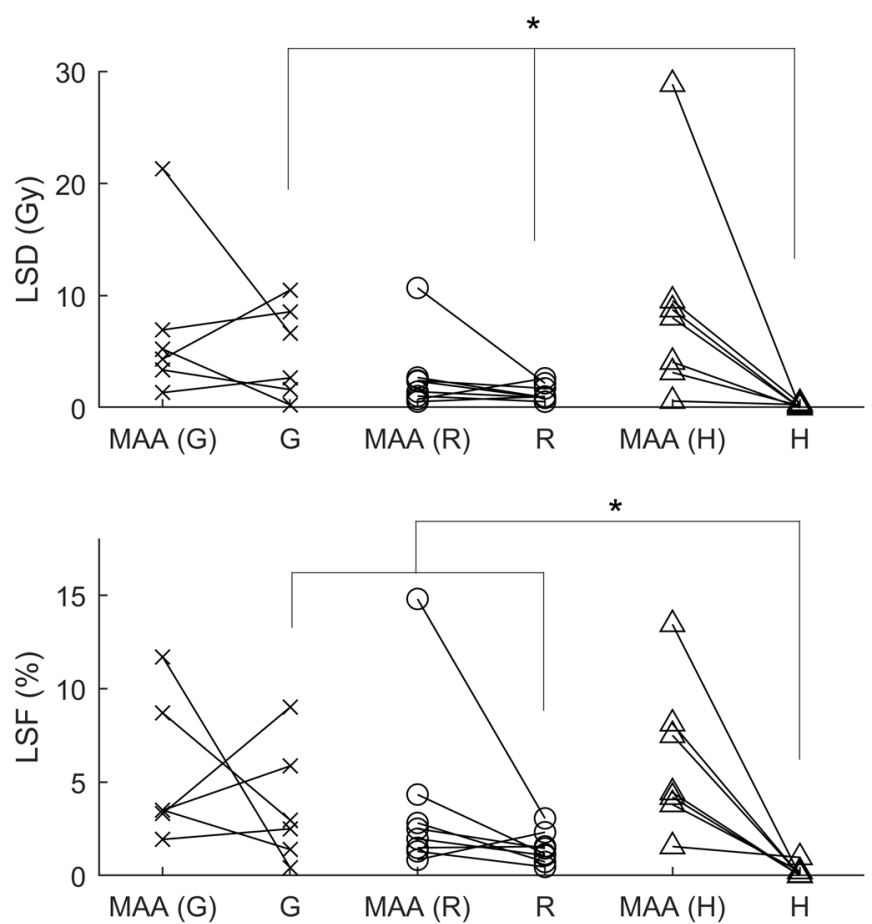

Figure 4.3: Dosimetry of the lungs for different microspheres. Data of individual patients are connected by a line. $L S D=$ lung shunt absorbed dose, $L S F=$ lung shunt fraction, $G={ }^{\circ 0} Y$ glass microspheres, $R=$ 90 resin microspheres, $\mathrm{H}={ }^{166} \mathrm{Ho}$ microspheres, $\mathrm{MAA}=$ technetium-99m labelled macroaggregated albumin. denotes significant difference between groups $(p<0.05)$.

Anatomic response according to RECIST 1.1 could be assessed in 20 patients (one patient could not be evaluated because the patient developed intrahepatic oedema after treatment (Figure 4.4), and of one patient only one tumour could be evaluated because the other two tumours suffered from beam hardening artefacts caused by surgical clips). Metabolic response based on [ $\left.{ }^{18} \mathrm{~F}\right] \mathrm{FDG}$ PET/CT could be assessed in 16 patients (the remaining 5 patients did not receive 3 month follow-up [ ${ }^{18}$ F]FDG PET/CT). Table 4.4 shows the response on a per-lesion basis. Number of tumours included for RECIST 1.1 and TLG change response were 6 and 6 for ${ }^{90} Y$ glass, 22 and 9 for ${ }^{90} Y$ resin, and 11 and 11 for ${ }^{166} \mathrm{Ho}$, respectively. Based on RECIST 1.1 criteria, ${ }^{90} \mathrm{Y}$ glass, ${ }^{90} \mathrm{Y}$ resin and ${ }^{166} \mathrm{Ho}$ microspheres showed no significant differences in response. Based on the TLG change, the ${ }^{166 \mathrm{Ho}}$ microspheres differed significantly from the ${ }^{90} \mathrm{Y}$ glass microspheres, but not from the ${ }^{90} Y$ resin microspheres (mean TLG change of $21 \%$ for ${ }^{166} \mathrm{Ho},-80 \%$ for ${ }^{90} Y$ glass, and $-15 \%$ for ${ }^{90} Y$ resin; $p=0.02$ ). Overall, on a per-patient basis according to RECIST 1.1, 2/20 patients showed partial response, 14/20 showed stable disease, and 4/20 showed progressive disease.

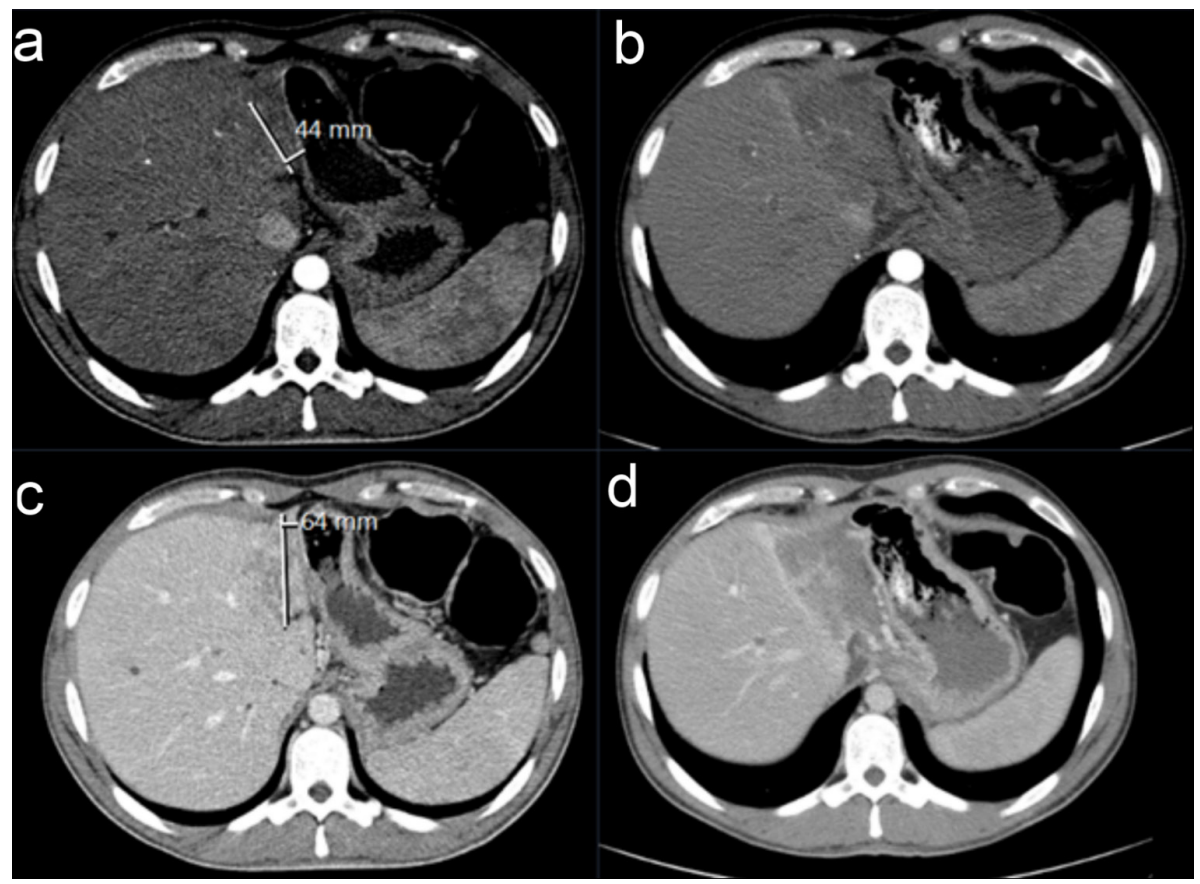

Figure 4.4: Extensive intrahepatic oedema and necrosis following treatment. Multiphase contrast-enhanced $C T$ at baseline $(a, c)$ and 3 months after left lobar treatment with $90 y$ glass microspheres $(b$, d). At baseline, the primary tumour invades the entire left liver lobe on the portal venous $C T$ (c) and shows mild enhancement in the arterial phase (a). After treatment the entire left liver lobe has intrahepatic radiation induced oedema and necrosis, making the primary tumour indistinguishable for response assessment according to RECIST 1.1. However, its contrast enhancement in the arterial phase has completely disappeared, in line with a complete response according to the modified RECIST (mRECIST).

Table 4.4: Response based on RECIST 1.1 and TLG change on a per-lesion basis

\begin{tabular}{lcccc}
\hline & & ${ }^{90} \mathbf{Y}$ glass & ${ }^{90}$ resin & ${ }^{166} \mathrm{Ho}$ \\
\hline \multirow{3}{*}{ RECIST 1.1 } & CR & $0 / 6(0 \%)$ & $0 / 22(0 \%)$ & $0 / 11(0 \%)$ \\
& PR & $1 / 6(17 \%)$ & $1 / 22(5 \%)$ & $2 / 11(18 \%)$ \\
& SD & $4 / 6(67 \%)$ & $16 / 22(73 \%)$ & $7 / 11(64 \%)$ \\
TLG change & PD & $1 / 6(17 \%)$ & $5 / 22(23 \%)$ & $2 / 11(18 \%)$ \\
& CR & $2 / 6(33 \%)$ & $2 / 9(22 \%)$ & $0 / 11(0 \%)$ \\
& PR & $4 / 6(67 \%)$ & $4 / 9(44 \%)$ & $3 / 11(27 \%)$ \\
& SD & $0 / 6(0 \%)$ & $2 / 9(22 \%)$ & $4 / 11(36 \%)$ \\
& PD & $0 / 6(0 \%)$ & $1 / 9(11 \%)$ & $4 / 11(36 \%)$ \\
\hline
\end{tabular}

$C R=$ complete response, $P R=$ partial response, $S D=$ stable disease, $P D=$ progressive disease, RECIST = response evaluation criteria in solid tumours, TLG = total lesion glycolysis 
Figure 4.5 shows the hepatotoxicity classification with respect to whole healthy liver absorbed dose and treated healthy liver absorbed dose. ${ }^{90} \mathrm{Y}$ glass, ${ }^{90} \mathrm{Y}$ resin and ${ }^{166} \mathrm{Ho}$ microspheres showed no significant differences in REILD classification. One patient (four days post-calibration ${ }^{90} \mathrm{Y}$ glass microspheres) had fatal REILD (grade 5), and this patient received the highest mean whole healthy liver absorbed dose (81 Gy). Furthermore, this patient showed one of the lowest T/N (0.6), although tumour targeting based on [99mTC] TC-MAA SPECT/CT was considered to be good. During the treatment angiography coil embolization of the segment 1 artery was performed, because of the development of a new collateral, which caused extrahepatic contrast enhancement of the lower oesophagus. Unfortunately, in retrospect, this artery also supplied a large portion of the tumour. This probably resulted in the low $\mathrm{T} / \mathrm{N}$, high whole healthy liver absorbed dose, and subsequent grade 5 REILD.
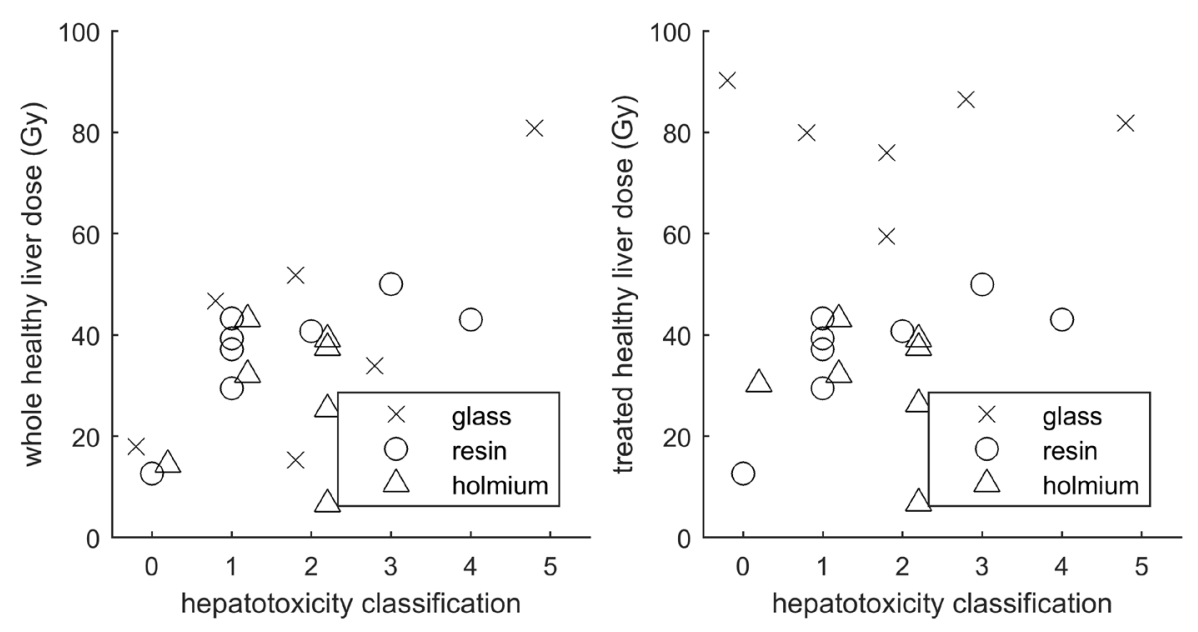

Figure 4.5: Hepatotoxicity classification with respect to whole healthy liver absorbed dose (left) and treated healthy liver absorbed dose (right).

\section{Discussion}

To our knowledge, this is the first case series comparing three commercially available microspheres for hepatic radioembolization treatment in locally advanced ICC. We showed differences in dosimetry between the three particles, where the ${ }^{90} Y$ glass microspheres delivered the highest absorbed doses. Nevertheless, the particles showed similar toxicity and response.
An important difference between the three microspheres is their specific activity. This results in large differences in the number of microspheres that are administered. Recently, Pasciak et al. showed that increasing the number of microspheres results in a more homogeneous dose distribution, leading to a greater volume fraction of the treated liver receiving a potentially toxic absorbed dose [134]. This could explain the differences observed in normal tissue complication probability found between ${ }^{90} \mathrm{Y}$ glass and ${ }^{90} \mathrm{Y}$ resin microspheres, where the smaller number of ${ }^{90} \mathrm{Y}$ glass microspheres show lower toxicity than ${ }^{90} Y$ resin microspheres at the same average absorbed dose $[123,135]$. The newer ${ }^{166} \mathrm{Ho}$ microspheres compare most to the ${ }^{90} \mathrm{Y}$ resin microspheres when it comes to the number of microspheres that are administered. The number of administered ${ }^{90} \mathrm{Y}$ glass microspheres is considerably less. However, care needs to be taken regarding the long shelf life of ${ }^{90} Y$ glass microspheres, that allows these microspheres to be used over a wide range of specific activities, and thus, number of administered microspheres. This needs to be considered during treatment planning. As different types of microspheres have different toxicity thresholds, this may also hold for ${ }^{90} \mathrm{Y}$ glass microspheres used at different days post-calibration.

The ${ }^{90} \mathrm{Y}$ glass microspheres delivered a higher tumour absorbed dose compared to the other particles. This difference was caused by two distinct differences: (i) a higher total amount of activity was administered with respect to the ${ }^{90} \mathrm{Y}$ resin microspheres (which is in line with other studies $[136,137])$, and (ii) all patients treated with glass microspheres received a partial liver treatment (e.g. lobar treatment or radiation segmentectomy). This results in a low whole healthy liver absorbed dose, while the treated healthy liver absorbed dose can be relatively high. The ${ }^{90} Y$ resin microsphere group consisted solely of whole liver treatments. Therefore, for this group, the whole healthy liver absorbed dose equals the treated healthy liver absorbed dose. The ${ }^{166} \mathrm{Ho}$ microsphere group consisted of a combination of whole liver and partial liver treatments.

Looking at response on a per-tumour basis, TLG change showed overall better response than RECIST 1.1. This was at least partially due to the fact that RECIST 1.1 does not distinguish between necrotic and non-necrotic tissue. Although we could not set a target dose for response based on these results, Figure 4.1 does show an example of a dose-response relationship within a single tumour. Figure 4.1 shows a residual tumour on follow-up 2-[18F]FDG PET/CT, corresponding to the part of the tumour receiving the lowest absorbed dose, as shown on ${ }^{90} \mathrm{Y}$ PET/CT.

In line with previous studies on radioembolization in ICC $[117,120,137,138]$, limited hepatotoxicity was found. Interestingly, grade 5 REILD occurred once in a patient who received by far the highest mean whole healthy liver absorbed dose ( $81 \mathrm{~Gy}$, where the $2^{\text {nd }}$ 
to highest whole healthy liver absorbed dose was only $52 \mathrm{~Gy}$ ). The treated healthy liver absorbed dose can be relatively high without inducing severe hepatotoxicity, as long as it relates to a partial liver treatment, so the whole healthy liver absorbed dose stays relatively low (Figure 4.5).

Planar scintigraphy images of MAA showed similar lung dosimetry for the entire population. This was expected [120,136,137], and confirms the similarity of the three groups at baseline. However, post-treatment lung dosimetry did show differences between therapeutic particles, where ${ }^{166 \mathrm{Ho}}$ microsphere stood out for both LSF and LSD. This is at least partially due to the different imaging modalities. ${ }^{166} \mathrm{Ho}$ images are acquired on a SPECT system, and the Monte Carlo reconstruction method used was able to perform accurate scatter correction, resulting in an accurate estimation of the LSF [61]. However, ${ }^{90} \mathrm{Y}$ images were acquired on a PET system, which suffers from both a low count rate and a high random fraction. This is known to cause a positive bias in the reconstruction, especially in parts of low counts (e.g. the lungs), resulting in an overestimation of the LSF [139-141]. LSD was higher for the ${ }^{90} Y$ glass microspheres than for the ${ }^{90} Y$ resin microspheres because of the higher administered activity.

The ultimate goal of radiation treatment is to deliver as high as possible dose to the tumours while keeping the dose to the healthy liver as low as possible. This is partially captured by the $\mathrm{T} / \mathrm{N}$, which did not show significant differences between the particles in this study. However, Nezami et al. [136] did show differences in T/N between ${ }^{90} \mathrm{Y}$ glass and ${ }^{90} \mathrm{Y}$ resin microspheres for ICC patients, where they assume this difference was caused by the differences in microsphere characteristics. This finding could not be replicated in the current study.

Contrary to other articles on dosimetry in radioembolization, absorbed dose was calculated based on post-treatment imaging, not on the pre-treatment [ ${ }^{99 \mathrm{~m} T C] T C-M A A ~ S P E C T / C T . ~ I n ~}$ addition, the reconstructed activity was not scaled based on the net administered activity. This introduces errors of ${ }^{90} \mathrm{Y} \mathrm{PET/CT}$ and ${ }^{166} \mathrm{HO}$ SPECT/CT quantification. However, it avoids errors introduced by scaling and residual activity measurement used to determine the administered net activity [142].

A limitation of this study is the small study population. In addition, follow-up 2-[18F]FDG PET/CT was not available for all patients. Also, modified RECIST could not be assessed in the majority of patients because of hypovascular tumours, and was therefore not assessed. This prevented us from drawing conclusions on dose-response and dose-toxicity relationships. However, looking at the entire population, irrespective of the particle used, and on a per-patient basis, the results were in line with literature $[117,120,137]$.
Another limitation was the bias in the groups regarding type of treatment. In our institute, all particles are used for whole liver treatments, but for selective treatments with a small target volume ${ }^{90} Y$ glass microspheres are preferred by the treating physicians, because of the high specific activity. Overall, in the presented locally advanced ICC patients, this led to a ${ }^{90} \mathrm{Y}$ glass group with only partial liver treatments, a ${ }^{90} \mathrm{Y}$ resin group with only whole liver treatments, and a mixed ${ }^{166} \mathrm{Ho}$ microsphere group. Additionally, we could not use the $\left.{ }^{999 m} \mathrm{Tc}\right] \mathrm{TC}-\mathrm{MAA}$ SPECT/CT data to compare intrahepatic dose metrics with posttreatment PET/CT or SPECT/CT. This was because of the high number of patients that had discrepancies between the MAA procedure and the therapeutic treatment (e.g. different injection positions, additional coiling) [34]. We did use the [99m Tc]Tc-MAA planar data for lung dosimetry, as a different catheter position would barely influence these results.

In the present study, we compared three commercially available microspheres in locally advanced ICC. These particles differ in physical characteristics and methods for calculating the prescribed activity. These differences lead to differences in absorbed dose, where ${ }^{90} \mathrm{Y}$ glass microspheres deliver the highest absorbed doses. Nevertheless, these groups show similar toxicity and response. This emphasises the need for separate dose constraints and dose targets for each particle. 


\section{Radioembolization lung shunt}

estimation based on a ${ }^{90} \mathrm{Y}$

pre-treatment procedure:

a phantom study

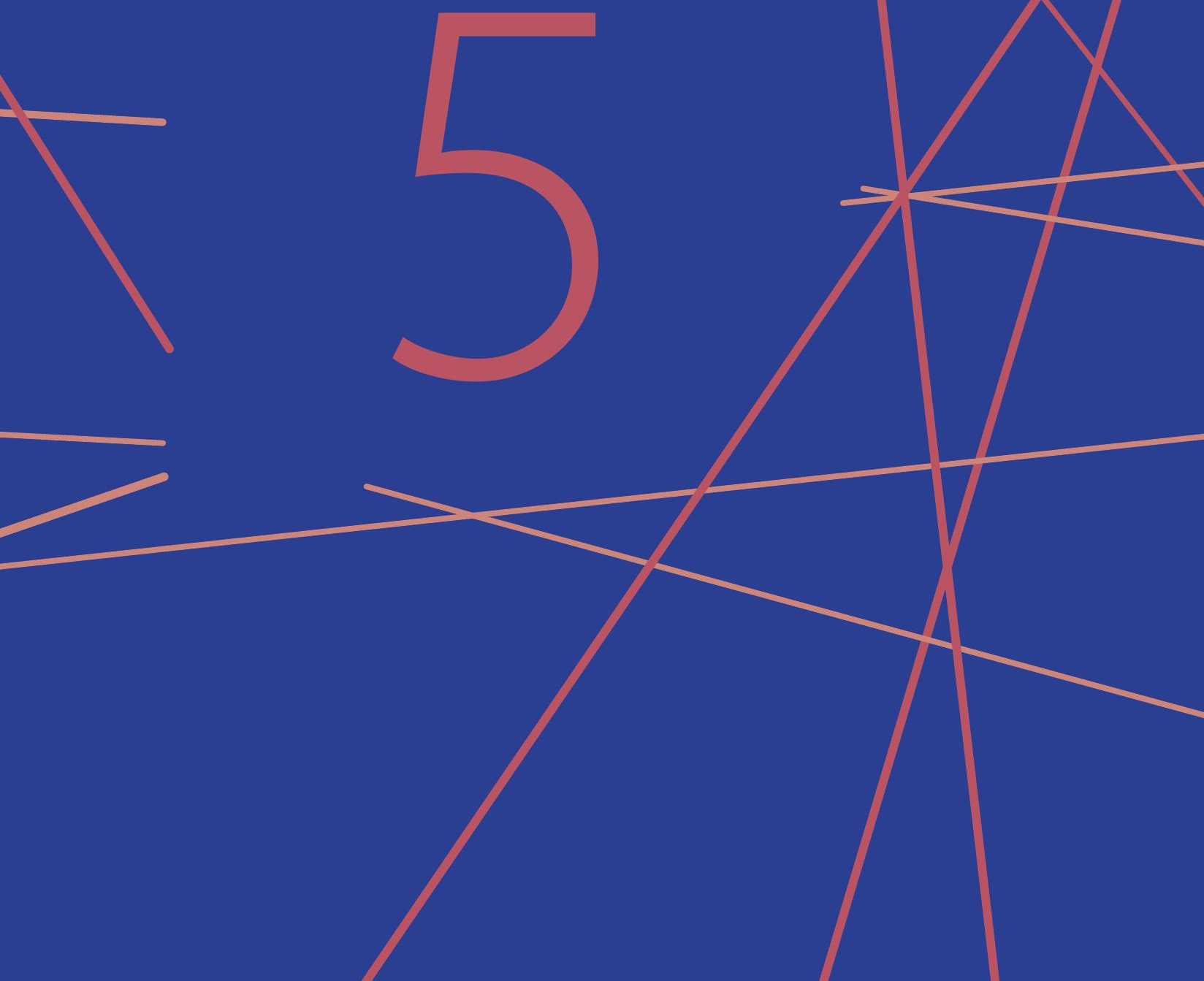




\section{Abstract}

Purpose: Prior to ${ }^{90} \mathrm{Y}$ radioembolization, a pre-treatment procedure is performed, in which

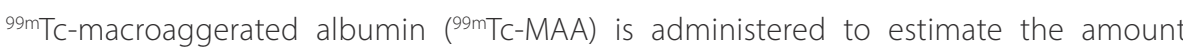
of activity shunting to the lungs. A high lung shunt fraction (LSF) may impose lower prescribed treatment activity or even impede treatment. Accurate LSF measurement is therefore important, but is hampered by the use of MAA particles, which differ from ${ }^{90} \mathrm{Y}$ microspheres. Ideally, ${ }^{90} \mathrm{Y}$ microspheres would also be used for the pre-treatment procedure, but this would require the activity to be lower than an estimated safety threshold of about $100 \mathrm{MBq}$ to avoid unintended radiation damage. However, ${ }^{90} \mathrm{Y}$ is very challenging to image, especially at low activities ( $<100 \mathrm{MBq}$ ). The purpose of this study was to evaluate the performance of three nuclear imaging techniques in estimating the LSF in a low activity ${ }^{90} Y$ pre-treatment scan, using an anthropomorphic phantom: (i) positron emission tomography/computed tomography (PET/CT), (ii) Bremsstrahlung single photon emission tomography/computed tomography (SPECT/CT), and (iii) planar imaging.

Methods: The lungs and liver of an anthropomorphic phantom were filled with ${ }^{90} \mathrm{Y}$ chloride to acquire an LSF of 15\%. Several PET/CT (Siemens Biograph mCT), Bremsstrahlung SPECT/ CT (Siemens Symbia T16) and planar images (Siemens Symbia T16) were acquired at a range of ${ }^{90} \mathrm{Y}$ activities (1586 MBq down to $25 \mathrm{MBq}$ ). PET images were reconstructed using a clinical protocol (attenuation correction, TOF, scatter and random correction, OP-OSEM), SPECT images were reconstructed using both a clinical protocol (attenuation correction, OSEM) and a Monte Carlo (MC) based reconstruction method (MC-based detector, scatter and attenuation modelling, OSEM), for planar images the geometric mean was calculated. In addition, in all cases except clinical SPECT, background correction was included. The LSF was calculated by assessing the reconstructed activity in the lungs and in the liver, as delineated on the CT images. In addition to the 15\% LSF, an extra 'cold' region was included to simulate an LSF of $0 \%$.

Results: PET reconstructions accurately estimated the LSF (absolute difference <2 percent point (pp)) when total activity was over $200 \mathrm{MBq}$, but greatly overestimated the LSF (up to 25pp) when activity decreased. Bremsstrahlung SPECT clinical reconstructions overestimated the LSF (up to 13pp) when activity was both high and low. Similarly, planar images overestimated the LSF (up to 23pp). MC-based SPECT reconstructions accurately estimated the LSF with an absolute difference of less than 1.3pp for activities as low as $70 \mathrm{MBq}$.
Conclusions: Bremsstrahlung SPECT/CT can accurately estimate the LSF for a ${ }^{90} \mathrm{Y}$ pretreatment procedure using a theoretically safe ${ }^{90} \mathrm{Y}$ activity as low as $70 \mathrm{MBq}$, when reconstructed with an MC-based model.

\section{Introduction}

Radioembolization is an established treatment for unresectable liver tumours where ${ }^{90} \mathrm{Y}$ loaded microspheres are administered in the arterial vasculature of the liver. Before the

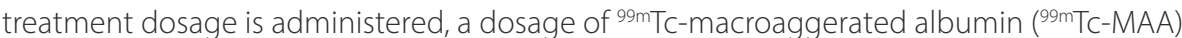
is administered to simulate ${ }^{90} \mathrm{Y}$ microsphere distribution [37]. We will refer to this procedure as the pre-treatment procedure. A single photon emission tomography/computed tomography (SPECT/CT) of the ${ }^{99 m} \mathrm{mc}-\mathrm{MAA}$ is acquired to detect potential extrahepatic depositions, and the lung shunt fraction (LSF) is estimated based on planar scintigraphy. However, the accuracy of this LSF estimation is uncertain and may be improved by using SPECT/CT images instead of planar scintigraphy [37-40]. Other problems regarding the accuracy of the LSF estimation still remain. First, the LSF may be overestimated by the MAA particles because of free circulating pertechnetate [44]. Second, the accuracy and reproducibility of the LSF estimation is thought to be affected by differences in shape and size distribution between MAA particles and microspheres [42,143]. These problems could be solved by using a particle for the pre-treatment procedure that is identical to the particle used for treatment, as is the essence of the ${ }^{166} \mathrm{Ho}$ microsphere treatment approach [144].

We have investigated the possibility of using ${ }^{90} \mathrm{Y}$ microspheres for the pre-treatment procedure to improve the estimation of the LSF for ${ }^{90} Y$ radioembolization. However, since ${ }^{90} \mathrm{Y}$ is an almost pure beta-emitter, it raises some concerns with regard to the safety of using it for the pre-treatment procedure. Low activities are preferred to avoid unintended radiation damage. For ${ }^{166} \mathrm{Ho}$ radioembolization, a pre-treatment procedure using an activity of $250 \mathrm{MBq}$, which is imaged by planar scintigraphy and SPECT/CT, is currently used in clinical studies and has been shown safe [26,144-146]. The total energy absorbed from the decay of $1 \mathrm{MBq}$ of ${ }^{166} \mathrm{Ho}$ is $15.9 \mathrm{~mJ}$, whereas the total energy absorbed from the decay of $1 \mathrm{MBq}$ of ${ }^{90} \mathrm{Y}$ is $49.4 \mathrm{~mJ}[29,145]$. Because the energy deposition of ${ }^{90} \mathrm{Y}$ is 3.11 times higher than that of ${ }^{166} \mathrm{Ho}$, theoretically a lower activity for ${ }^{90} \mathrm{Y}$ pre-treatment procedures should be used to be as safe as the current clinical ${ }^{166} \mathrm{Ho}$ protocol, for example an activity around 80-100 MBq. This low activity ( $<100 \mathrm{MBq}$ ) imposes some difficulties for imaging.

${ }^{90} \mathrm{Y}$ imaging with positron emission tomography (PET) is based on the small decay branch of ${ }^{90} Y$ generating positrons. Only 32 in a million decays result in positron emission [147]. This small positron decay ratio is sufficient for post-treatment dosimetry when patients 
receive a high amount of activity (treatment), but it may not be sufficient to accurately estimate the LSF when the activity is strongly reduced (pre-treatment procedure) [38] ${ }^{90} \mathrm{Y}$ PET accuracy under low count conditions has been investigated with phantom studies [143,148-151]. These studies focused on lesion detectability, recovery coefficients, reconstruction algorithms and image acquisition parameters. However, to estimate the LSF, one must quantify very low activity concentrations. Activity recovery of a low concentration (37 kBq/mL) of ${ }^{90} \mathrm{Y}$ has been described by Willowson et al. [148]. However, for a pre-treatment procedure one needs to be able to measure even lower activity concentrations, especially in the lung area $(<10 \mathrm{kBq} / \mathrm{mL}$, based on an activity of $100 \mathrm{MBq}$, maximum LSF of $20 \%$ and a lung volume of $2 \mathrm{~L}$ ).

Another possibility for ${ }^{90} \mathrm{Y}$ imaging is SPECT based on Bremsstrahlung photons Bremsstrahlung photons have a very broad and continuous spectrum which prohibits the use of scatter windows for scatter correction. Scatter correction for Bremsstrahlung SPECT therefore relies on time-consuming Monte Carlo (MC) reconstruction models. Although there is general consensus that ${ }^{90} \mathrm{Y} \mathrm{PET}$ produces superior image quality over Bremsstrahlung SPECT [152], Bremsstrahlung SPECT is more sensitive than ${ }^{90} \mathrm{Y} \mathrm{PET} \mathrm{and}$ might therefore be better suited to estimate low activities.

In this study an anthropomorphic phantom was used to investigate whether the LSF of a ${ }^{90} \mathrm{Y}$ pre-treatment procedure can be accurately determined by ${ }^{90} \mathrm{Y} \mathrm{PET/CT}$, Bremsstrahlung SPECT/CT, and/or planar scintigraphy.

\section{Materials and Methods}

\section{Phantom}

We used an anthropomorphic thorax phantom (Radiology Support Devices, Long Beach, CA), which represents the torso of an average male (Figure 5.1). The phantom was designed to realistically simulate PET and SPECT acquisitions. It includes the bone structures within the torso and fillable compartments representing the liver and lungs.

The liver $(1.1 \mathrm{~L})$ was filled with an initial activity of $1347 \mathrm{MBq}$, as measured by a dose calibrator (VDC-404, Veenstra Instruments), of ${ }^{90} \mathrm{Y}$ chloride in $0.5 \mathrm{M}$ of $\mathrm{HCl}$ to prevent adhesion to the plastic phantom walls [153]. The lungs, filled with styrofoam beads, were filled with a total initial activity of $239 \mathrm{MBq}$ of ${ }^{90} \mathrm{Y}$ chloride in $0.5 \mathrm{M}$ of $\mathrm{HCl}$. The total volume of the lungs was $2.0 \mathrm{~L}$ and their density was $0.40 \mathrm{~g} / \mathrm{cm}^{3}$. The remainder of the thorax phantom was filled with water. The total initial activity of the phantom, $1586 \mathrm{MBq}$, was comparable to a clinically used treatment dosage of ${ }^{90} \mathrm{Y}$ resin microspheres (SIR-Spheres ${ }^{\varpi}$,
Sirtex) $[124,154]$. The phantom was imaged multiple times during decay, down to a final total activity of $25 \mathrm{MBq}$, which is well below the estimated safety threshold of about 100 $\mathrm{MBq}$ for a ${ }^{90} \mathrm{Y}$ pre-treatment procedure.

The LSF was defined as the activity in the lungs divided by the activity in the lungs and the liver [37]. Resin microspheres are contraindicated in patients who have an LSF of more than $20 \%$. It is advocated to reduce the prescribed activity in patients who have an LSF between 10\% and 20\% [127]. Glass microspheres (TheraSphere ${ }^{\oplus}$ BTG) are contraindicated in patients whose LSF could result in a delivery of more than $610 \mathrm{MBq}$ of ${ }^{90} Y$ to the lungs (i.e. approximately 30 Gy for 1 kg lungs) [25]. The LSF of our phantom was 15\%. This LSF was chosen because it is within the range where LSF-indicated activity reductions are generally advocated; therefore, the LSF needs to be accurately estimated within this range.

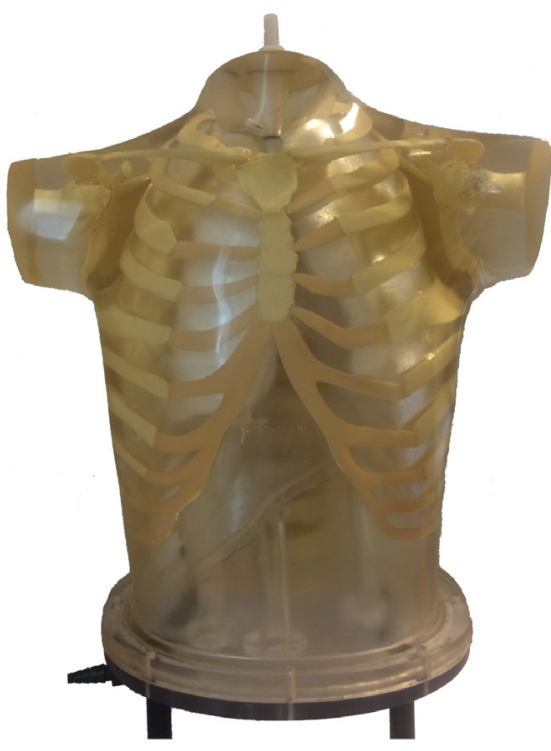

Figure 5.1: Anthropomorphic thorax phantom with ung and liver inserts.

\section{Image acquisition}

Twelve PET/CT scans, twelve SPECT/CT scans and twelve planar scintigraphy images were acquired over a period of six half-lives to include a broad range of ${ }^{90} \mathrm{Y}$ activities $\left(\mathrm{t}_{1 / 2}=64.1\right.$ hours) (Table 5.1).

\section{Table 5.1: Phantom activities at time of acquisition}

\begin{tabular}{lcccccccccccc}
\hline Scan number & $\mathbf{1}$ & $\mathbf{2}$ & $\mathbf{3}$ & $\mathbf{4}$ & $\mathbf{5}$ & $\mathbf{6}$ & $\mathbf{7}$ & $\mathbf{8}$ & $\mathbf{9}$ & $\mathbf{1 0}$ & $\mathbf{1 1}$ & $\mathbf{1 2}$ \\
\hline $\begin{array}{l}\text { Total phantom activity at PET } \\
\text { acquisition (MBq) }\end{array}$ & 1570 & 946 & 557 & 336 & 220 & 100 & 89 & 70 & 54 & 42 & 35 & 25 \\
$\begin{array}{l}\text { Total phantom activity at SPECT } \\
\text { acquisition (MBq) }\end{array}$ & 1550 & 959 & 56 & 3411 & 223 & 119 & 88 & 70 & 55 & 42 & 35 & 26 \\
$\begin{array}{l}\text { Total phantom activity at planar } \\
\text { scintigraphy (MBq) }\end{array}$ & 1524 & 952 & 561 & 338 & 221 & 118 & 87 & 69 & 54 & 42 & 35 & 26 \\
\hline
\end{tabular}


PET/CT images were acquired on a Siemens Biograph mCT time of flight (TOF) system. Three bed positions were scanned to fit the entire phantom in the field of view (FOV). Acquisition time was 15 minutes per bed position (in agreement with our clinical protocol [100]) (total acquisition time of 45 minutes) and consecutive bed positions overlapped approximately 43\%. A CT scan (120 kVp, 39 mAs) was made for attenuation correction and to support organ delineation.

SPECT/CT and planar images were acquired on a dual head Siemens Symbia T16 system For the SPECT/CT scans the lungs and liver of the phantom fit in a single FOV. Data were acquired in a 50-250 keV energy window with the high-energy collimators mounted [16]. Projections were acquired for 30 seconds per angle, using 120 angles over 360 degrees (total acquisition time of 30 minutes). These acquisition parameters were identical to our clinical settings [100]. A CT scan (110 kVp, $30 \mathrm{mAs})$ was made for attenuation correction and to support organ delineation.

Planar scintigraphy images were acquired by means of a whole body scan with a scanning speed of $5 \mathrm{~cm} / \mathrm{min}$ (total acquisition time of 20 minutes). The two gamma cameras were positioned opposite each other to acquire anterior and posterior images. Data were acquired in a 50-250 keV energy window with the high-energy collimators mounted.

For both SPECT and planar acquisitions a single background measurement was performed without the phantom present using the settings described above. For PET a single long background measurement (total scan time of 24 hours) was performed with the phantom without activity in the scanner.

\section{Image reconstruction}

For each imaging modality, data was reconstructed using the clinical protocol of our institution. In addition, a background correction was performed for each modality.

For PET data, the first reconstruction method (PET-clinical) was a clinically used reconstruction algorithm on the scanner console. This is an ordinary Poisson ordered subset expectation maximization (OP-OSEM) reconstruction method, including resolution recovery (TrueX), TOF information, random, attenuation, scatter, dead time, and decay correction. Random correction was based on smoothed delayed coincidences, Attenuation correction was based on a CT scan. Images were reconstructed using 4 iterations with 21 subsets and a $5 \mathrm{~mm}$ full width at half maximum (FWHM) Gaussian post-reconstruction filter was applied. The reconstructed voxel size was $4.1 \times 4.1 \times 3.0$ $\mathrm{mm}^{3}$. Because ${ }^{90} \mathrm{Y}$ is not provided on the isotope list of the scanner, ${ }^{89} \mathrm{Zr}$ was used as an alternative isotope for acquisition and reconstructed images were corrected for the different half-life and positron branching ratio. The second method (PET-BGcorr) included a background correction in which the activity in the volumes of interest (VOIs) of the background image (reconstructed as described above) was subtracted from the activity in the VOls of the phantom image.

For SPECT data, the first reconstruction method (SPECT-clinical) was a clinically used twodimensional OSEM (2D OSEM) algorithm using 8 iterations with 5 subsets. Attenuation correction was based on a CT scan. No scatter correction or post-reconstruction filter was applied. The reconstructed voxel size was $2.4 \times 2.4 \times 2.4 \mathrm{~mm}^{3}$. The second algorithm (SPECT-MC) was an MC-based OSEM algorithm. In this reconstruction method scatter, attenuation, and collimator-detector response were MC simulated. This reconstruction method and its validation are described in detail by Elschot et al. [17]. Since MC-based reconstructions take longer to converge, images were reconstructed using 60 iterations and 8 subsets [17]. No post-reconstruction filter was applied. The reconstructed voxel size was $4.8 \times 4.8 \times 4.8 \mathrm{~mm}^{3}$. The third algorithm (SPECT-MC-BGcorr) was the same as the SPECT-MC algorithm, but in addition included a background correction in this MC reconstruction algorithm by adding the average measured background count to each forward projection.

Planar images were created from the geometric means of the anterior and posterior images and the pixel size was $2.4 \times 2.4 \mathrm{~mm}^{2}$ (planar-clinical). Background correction was performed by subtracting the counts in the regions of interest (ROIs) of the background image from the counts in the ROls of the phantom image (planar-BGcorr).

\section{Analysis}

The lungs and the liver were manually delineated on one CT scan to create a lung and a liver volume of interest ( $\mathrm{VOI}$ ). This reference $\mathrm{CT}$ was rigidly registered to all other $\mathrm{CT}$ scans and the $\mathrm{VOI}$ of the liver was transformed accordingly to ensure that the same volume was analysed for each scan. In between scans, during transport, the lungs could slightly move within the phantom which could induce slight differences in position for each scan. Therefore, for each lung, a separate rigid registration from the reference $\mathrm{CT}$ to all other CTs was performed. VOIs of the lungs were transformed accordingly. All registrations and transformations were performed using Elastix and visually inspected [129].

To partly compensate for partial volume effects, the VOls were dilated by the spatial resolution of each system, $6 \mathrm{~mm}$ for PET and $14 \mathrm{~mm}$ for SPECT. When this dilation resulted in overlapping VOIs, the liver VOI had priority over the lung VOI. This approach was equal to what is used in clinical LSF analysis. VOls were transferred to the PET and SPECT reconstructions and the LSF was calculated by dividing the activity in the lung 
VOI by the activity in the lung and liver VOIs. The recovery coefficient was calculated by dividing the observed activity by the true activity. Because SPECT and planar images hold counts instead of $\mathrm{Bq} / \mathrm{mL}$ like PET, the observed activity for these modalities is based on the known total activity in the phantom as:

$$
\mathrm{A}_{\mathrm{ROI}}=\frac{\mathrm{A}_{\text {tot }}}{\mathrm{C}_{\text {lung }}+\mathrm{C}_{\text {liver }}} \mathrm{C}_{\mathrm{ROI}} \text {, }
$$

where $A_{\text {ROI }}$ is the observed activity in either the lung or liver ROI, $A_{\text {tot }}$ is the total activity of the phantom at the time of imaging, Clung is the amount of lung counts, $C_{\text {liver }}$ is the amount of liver counts and $C_{\text {ROI }}$ is the amount of counts in either the lung of the liver ROI.

The LSF of the anthropomorphic phantom in this study was 15\%. However, the majority of radioembolization patients have an LSF $<10 \%$ [124]. These patients should be identified as suitable for treatment without the need of dosage reduction. To examine the accuracy of the LSF estimations for low LSFs, an extra region, apart from the lungs and the liver, was delineated on the reference $C T$. This region was a large $(1.5 \mathrm{~L})$, fully connected VOI located below the liver that fitted inside the FOV of all scans (Figure 5.2). It held cold (no activity present) water and served to simulate lungs with no activity (LSF of 0\%). This cold VOI was transformed to the other $\mathrm{CT}$ scans according to the liver transformations previously described. The cold VOI had a smaller volume than the lung VOI, which leads to a smaller LSF estimate for equal activity concentrations. To compensate for this volume difference, the activity in the cold VOI was scaled to the lung volume by multiplying the activity in the cold VOI by the fraction of lung VOI volume over cold VOI volume.

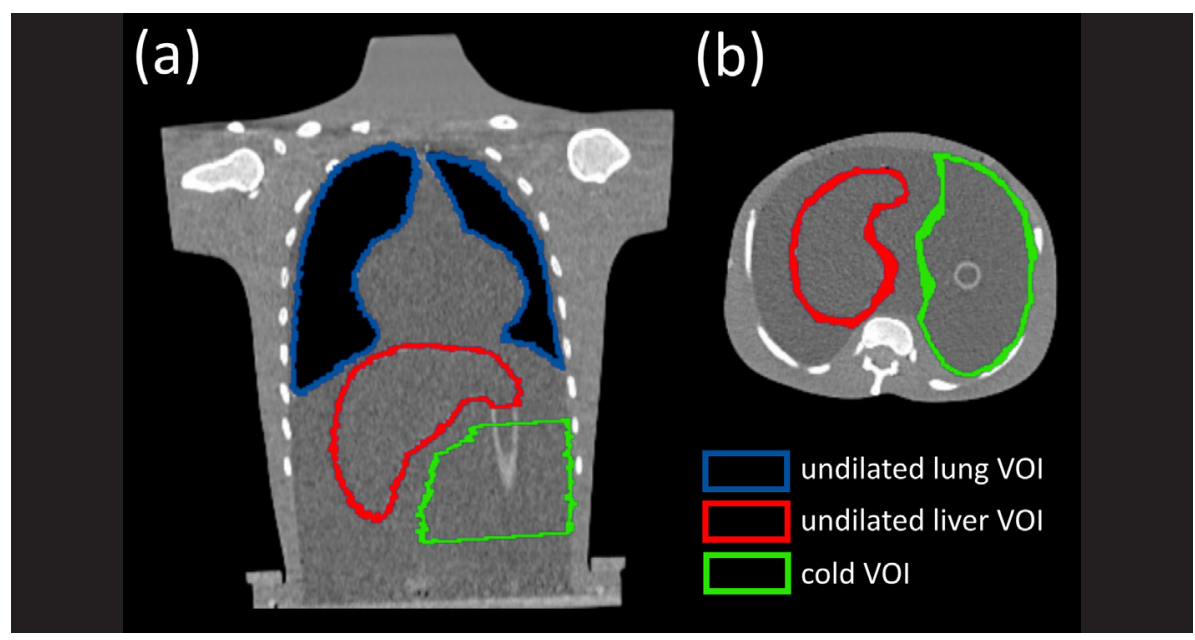

Figure 5.2: Coronal (a) and axial (b) views of a CT of the anthropomorphic thorax phantom with the undilated lung VOI (blue), the undilated liver VOI (red), and the cold VOI (green).
For planar images, all previously described 3D VOls from the corresponding SPECT/CT scans were projected to form $2 \mathrm{D}$ ROIs. Since the planar images had no reference to the CT scans, the liver ROI was manually positioned over the hot liver region in the planar image, which was clearly visible on all images. This approach was equal to what is used in clinical LSF analysis. The same transformation was then applied to the lung and cold ROls, because the phantom was not moved between the SPECT/CT and the planar acquisition. The LSF was calculated as described previously.

In order to get an estimate of the error margins on these LSF measurements we varied the dilation applied to the lung and liver VOls by $\pm 5 \mathrm{~mm}$. This represented the variation in delineation caused by different observers.

\section{Results}

The number of counts or true coincidences acquired during the different exams are listed in Table 5.2.

Figure 5.3 shows the coronal views of the PET and SPECT reconstructions and the planar images for scans with a total phantom activity of approximately 1550, 88 and $26 \mathrm{MBq}$. For total activities over $\sim 350 \mathrm{MBq}$, the PET reconstructions had a visually higher spatial resolution than the SPECT reconstructions. For total activities below 350 MBq the PET reconstructions were more noisy and below $\sim 70 \mathrm{MBq}$ the liver was no longer identifiable.

\begin{tabular}{|c|c|c|c|}
\hline & $\begin{array}{c}\text { Scan } 1 \\
(\sim 1550 \mathrm{MBq})\end{array}$ & $\begin{array}{c}\text { Scan } 12 \\
(\sim 26 \mathrm{MBq})\end{array}$ & $\begin{array}{c}\text { Background measurement } \\
\text { (0 MBq) }\end{array}$ \\
\hline $\begin{array}{l}\text { Counts acquired during SPECT exam } \\
\text { (30 minutes) }\end{array}$ & $1.9 \cdot 10^{7}$ & $6.0 \cdot 10^{5}$ & $2.8 \cdot 10^{5}$ \\
\hline $\begin{array}{l}\text { Counts acquired during planar } \\
\text { imaging (20 minutes) }\end{array}$ & $6.1 \cdot 10^{6}$ & $2.3 \cdot 10^{5}$ & $1.3 \cdot 10^{5}$ \\
\hline $\begin{array}{l}\text { True coincidences acquired during } \\
\text { PET exam (45 minutes) }\end{array}$ & $3.9 \cdot 10^{5}$ & $1.7 \cdot 10^{4}$ & $\begin{array}{c}3.0 \cdot 10^{5} \text { (24-hour exam) } \\
9.3 \cdot 10^{3} \text { (scaled to } 45 \text {-minute } \\
\text { exam) }\end{array}$ \\
\hline
\end{tabular}

SPECT-clinical reconstructed a hot liver for all scans, but with substantial spillover activity outside the liver. Part of this spillover activity was reconstructed inside the lungs, but there was no visual homogeneous activity reconstructed inside the lungs (the upper parts of the lungs had no reconstructed activity in any of the scans). 


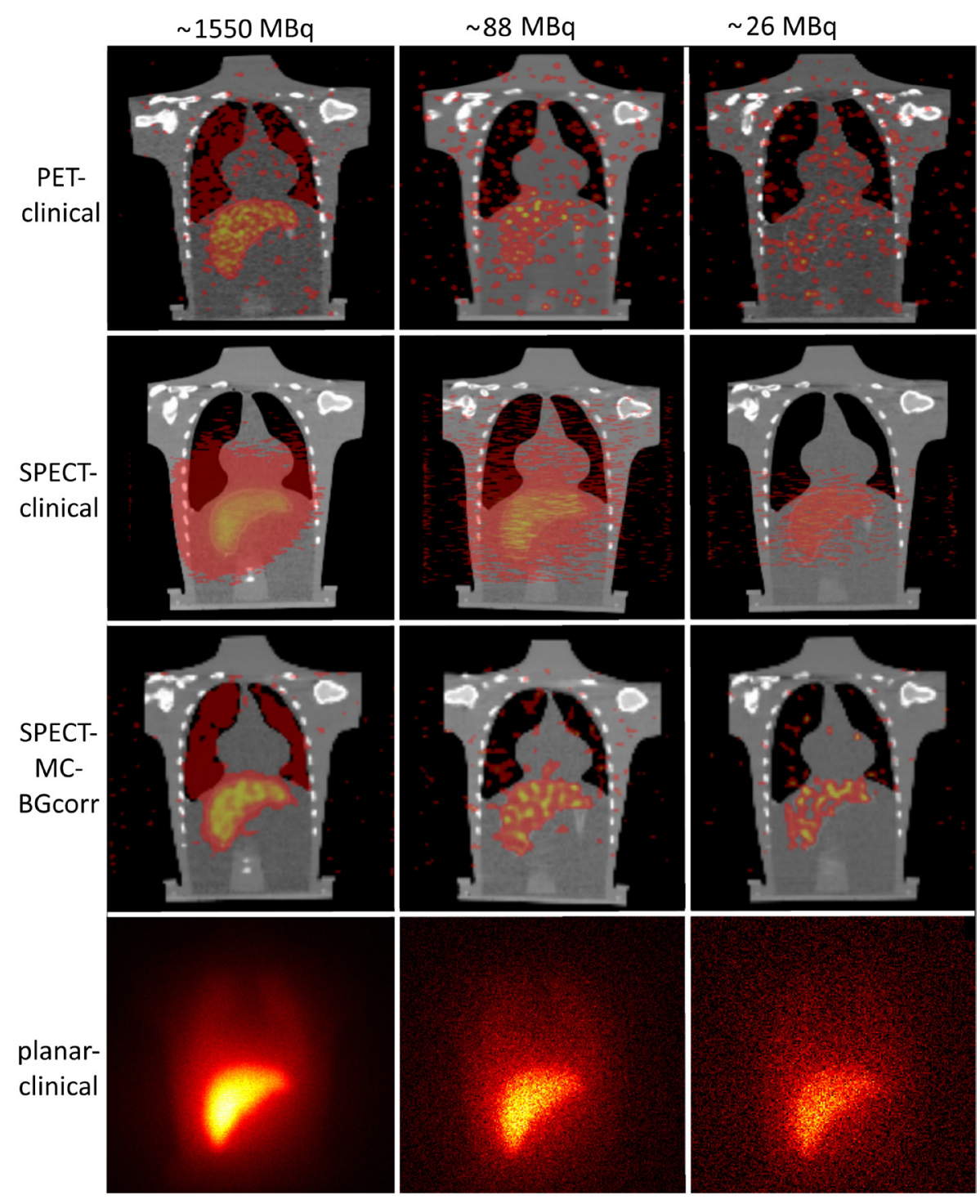

Figure 5.3: Coronal views of the PET-clinical, SPECT-clinical, and SPECT-MC-BGcorr reconstructions and geometric means of the planar-clinical images for scans with a total phantom activity of approximately 1550,88 and $26 \mathrm{MBq}$

SPECT-MC and SPECT-MC-BGCorr were visually very similar (SPECT-MC had slightly more noise than SPECT-MC-BGcorr). Both reconstructed uniform activity in both the liver and the lungs for total activities over $\sim 500 \mathrm{MBq}$. For total activities below $\sim 500$ $\mathrm{MBq}$, the reconstructions were more noisy, but in contrast to PET, the liver was still visible in all reconstructions.
Planar images showed the liver clearly, just like the SPECT-clinical reconstructions, but with substantial (scattered) background and spillover activity.

Figure 5.4 shows the activity recovery of the liver and the lungs for all imaging modalities. For PET, activity recovery was accurate (less than 10\% difference) for activities over 45 MBq, but activity was largely overestimated for activities below $35 \mathrm{MBq}$. This overestimation was slightly reduced by performing a background correction.
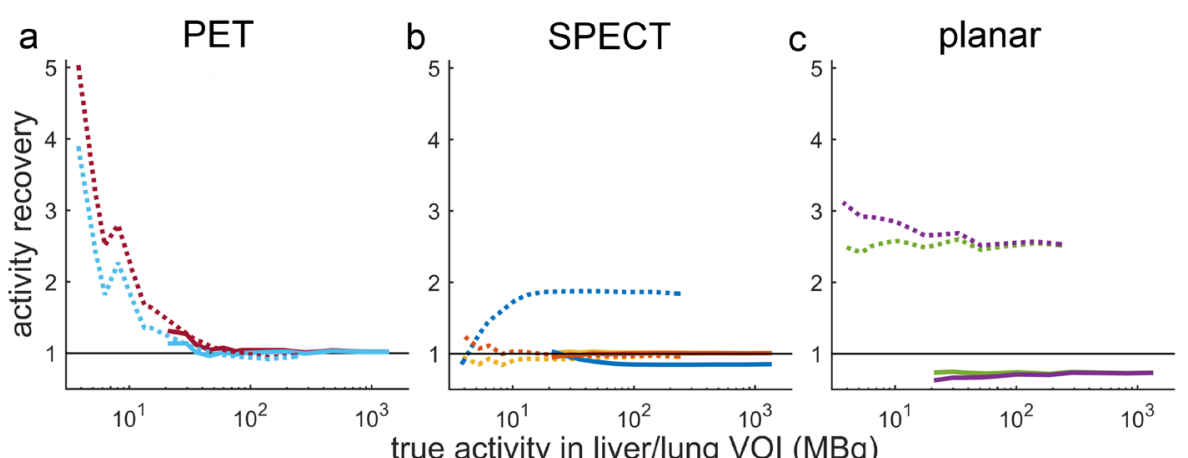

- liver PET-clinical

true activity in liver/lung $\stackrel{10^{2}}{10^{3}}(\mathrm{MBq})$ SPECT-clinical SPECT-MC

planar-clinical SPECT-MC-BGcorr

Figure 5.4: Activity recovery for the liver (solid lines) and the lungs (dotted lines), for PET (a), SPECT (b), and planar images (c). Recovery coefficients $>1$ indicate overestimation of true activity, recovery coefficient $<1$ indicate underestimation of true activity.

SPECT-clinical showed overestimation of activity in the lungs and underestimation of activity in the liver. The SPECT-MC reconstruction method showed large improvement over the SPECT-clinical reconstruction method. Using an MC-based reconstruction method led to accurate activity recovery (less than 10\% difference) for activities over $10 \mathrm{MBq}$. Including a background correction in the MC-based reconstruction method had most effect on the scans with a total activity below $100 \mathrm{MBq}$. The activity recovery of the SPECT-MC-BGcorr varied between 1.01 and 1.03 for the liver compartment and between 0.84 and 0.97 for the lung compartment. The activity recovery of the SPECT-MC varied between 0.95 and 1.01 for the liver compartment and between 0.96 and 1.26 for the lung compartment.

Planar images largely overestimated the activity in the lungs and underestimated the activity in the liver, for the full range of activities. The background correction made the activity recovery more stable over the range of activities, but did not completely correct for the over- and underestimation in the lungs and liver, respectively. 
Figure $5.5 \mathrm{a}, \mathrm{b}, \mathrm{c}$ shows the estimated LSF for a true LSF of 15\%. PET accurately estimated the LSF (absolute difference <2 percent point (pp)) for total activities over $200 \mathrm{MBq}$ (absolute difference of $0.6 \mathrm{pp}$ (PET-clinical) and $0.8 \mathrm{pp}$ (PET-BGcorr) at $1570 \mathrm{MBq}$ ), but started to overestimate (up to $25 \mathrm{pp}$ ) the LSF as the activity decreased. The background correction had most effect on scans with a total activity below $100 \mathrm{MBq}$. Furthermore, the LSF was more sensitive to varying delineations as the activity decreased, shown by the increasing shaded area.

SPECT-clinical overestimated the LSF for almost all activities (absolute difference of 13pp at $1550 \mathrm{MBq}$ ), as expected based on visual inspections of the reconstructions. Similarly, planar imaging largely overestimated the LSF for all scans (absolute difference between $21 \mathrm{pp}$ and $31 \mathrm{pp})$.
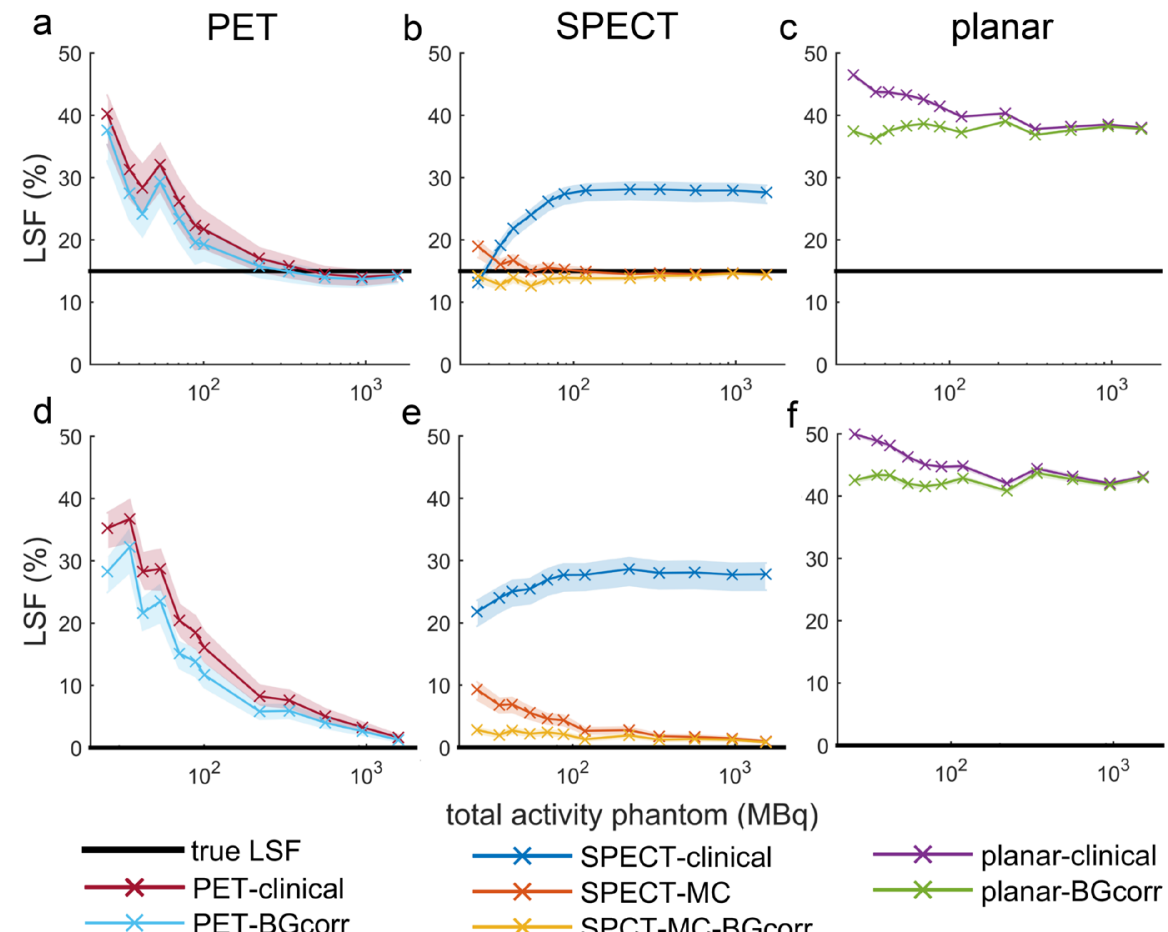

total activity phantom $(\mathrm{MBq})$

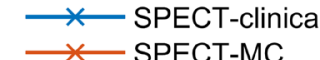
$\longrightarrow$ SPECT-MC

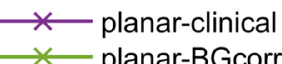

Figure 5.5: $\operatorname{LSF}$ for PET (a, d), $\operatorname{SPECT}(b, e)$, and planar images $(c, f)$ as a function of total ${ }^{90} \mathrm{Y}$ activity in the phantom. The horizontal black lines indicate the true LSF of the phantom, which is either $15 \%(a, b, c)$ or $0 \%$ $(d, e, f)$. Shaded areas show the uncertainty in the LSF caused by varying the dilation of the VOls by $\pm 5 \mathrm{~mm}$.
SPECT-MC and SPECT-MC-BGcorr both estimated the LSF accurately for total activities over $70 \mathrm{MBq}$ (absolute difference of 0.5pp (SPECT-MC) and 0.6pp (SPECT-MC-BGcorr) at $1550 \mathrm{MBq}$ and an absolute difference of 0.5pp (SPECT-MC) and 1.3pp (SPECT-MC-BGcorr) at $70 \mathrm{MBq}$ ). Furthermore, the uncertainty represented by the shaded area was quite small for total activities over $70 \mathrm{MBq}( \pm 0.9 \mathrm{pp}$ (SPECT-MC) and $\pm 0.5 \mathrm{pp}$ (SPECT-MC-BGcorr) at $70 \mathrm{MBq})$.

Figure $5.5 \mathrm{~d}$, e, f shows the LSF based on the cold VOI, simulating an LSF of 0\%. PET estimated the LSF accurately only for the scan with a total activity of $1570 \mathrm{MBq}$ (absolute difference of $1.7 p p$ (PET-clinical) and 1.3pp (PET-BGcorr) at $1570 \mathrm{MBq}$ ), but largely overestimated the LSF as the activity decreased (up to $35 \mathrm{pp}$ ).

Planar-clinical, planar-BGcorr and SPECT-clinical overestimated the LSF, similarly to the overestimation seen for the LSF of $15 \%$

SPECT-MC and SPECT-MC-BGcorr estimated the LSF accurately for total activities over $100 \mathrm{MBq}$ (absolute difference of 1.0pp (SPECT-MC) and 0.8pp (SPECT-MC-BGcorr) at 1550 $\mathrm{MBq}$ ). As the activity decreased, SPECT-MC-BGcorr outperformed SPECT-MC, having a more accurate LSF estimation (absolute difference of 2.4pp (SPECT-MC-BGcorr) vs. 4.6pp (SPECT-MC) at $70 \mathrm{MBq}$ ) and less uncertainty in its LSF estimation ( \pm 0.5pp (SPECT-MCBGcorr) vs. $\pm 0.8 p p(S P E C T-M C)$ at $70 \mathrm{MBq})$.

\section{Discussion}

This study evaluated the ability to accurately estimate the LSF of ${ }^{90} Y$-based radioembolization by means of a low activity ${ }^{90} \mathrm{Y}$-based pre-treatment procedure. The imaging methods investigated comprised PET/CT, Bremsstrahlung SPECT/CT and planar imaging. We found that Bremsstrahlung SPECT/CT, when reconstructed with an MC-based model including a background correction, estimated the LSF down to activities as low as $70 \mathrm{MBq}$ with a maximum absolute difference of 1.3 percentage points.

Since ${ }^{90} \mathrm{Y}$ is a high-energy beta-emitting radioisotope, it raises concerns with regard to the safety of using it for the pre-treatment procedure. Low concentrated activity in abdominal organs, such as the stomach, duodenum and pancreas, may result in a high absorbed dose with dismal consequences. Because of the low incidence of extrahepatic deposition of activity, no dose-effect relationships have been established, but the general consensus is that absorbed doses in abdominal organs are safe up to approximately 50 Gy [155]. Based on the empirically proven safety of a ${ }^{166} \mathrm{Ho}$ pre-treatment procedure using 
$250 \mathrm{MBq}$, and the fact that ${ }^{90} \mathrm{Y}$ has a 3.11 times higher energy deposition compared to ${ }^{166} \mathrm{Ho}$, theoretically, a lower activity for the ${ }^{90} \mathrm{Y}$ pre-treatment procedure should be used $[29,144,145]$. That would result in a ${ }^{90} \mathrm{Y}$ activity of around $80-100 \mathrm{MBq}$, which is above the threshold for accurate LSF estimation using Bremsstrahlung SPECT/CT when reconstructed with an MC-based reconstruction model. The use of $100 \mathrm{MBq}$ of ${ }^{90} \mathrm{Y}$ for the pre-treatment procedure could result in a maximum lung dose of 5 Gy (worst case scenario of 100\% LSF for $1 \mathrm{~kg}$ lungs). This is well below the safety threshold of $30 \mathrm{~Gy}$ to the lungs.

On the one hand low activities for pre-treatment procedures are preferred for safety reasons. On the other hand the activity should also be sufficient to observe any extrahepatic depositions. Prince et al. investigated 34 extrahepatic depositions from a group of 160 patients who received a ${ }^{99 m} \mathrm{mC}-M A A$ injection as part of their radioembolization workup [145]. The extrahepatic depositions had a median activity of $1.3 \%$ of the administered activity and a median volume of $6.8 \mathrm{~mL}$. For a ${ }^{90} \mathrm{Y}$ pre-treatment procedure using $80 \mathrm{MBq}$ this would result in an activity concentration of $153 \mathrm{kBq} /$ $\mathrm{mL}$. According to some studies, spheres with activity concentrations this low are not visible on PET/CT $[143,148,151]$. However, these studies were performed to investigate lesion detectability and the spheres in these studies had to be distinguishable from background activity. Extrahepatic depositions are generally located in a cold area, so visibility could be better since there is no background activity. To our knowledge, there are no studies describing the limits of lesion detectability in MC-based reconstruction models for Bremsstrahlung SPECT/CT. However, Dewaraja et al. did describe the visibility of extrahepatic depositions in a ${ }^{90} \mathrm{Y}$ phantom study in which their reconstruction model included MC-based scatter correction [18]. The lowest simulated extrahepatic activity concentration in their study was $108 \mathrm{kBq} / \mathrm{mL}$, and when the MC-based scatter correction was included in the reconstruction model, this extrahepatic deposition was clearly visible. While this result gives confidence in the potential of MC-based Bremstrahlung SPECT/CT for quantification of extrahepatic depositions, the visibility of such depositions with ${ }^{90} Y$ imaging needs further investigation.

The number of background counts acquired in the PET, SPECT and planar acquisition protocols were a small fraction of the total counts for the acquisitions with high activity, but the background counts take up a significant part of the total counts for the acquisitions with low activities(<100 MBq). For these low activities, background correction had a significant impact on LSF estimation.

The SPECT-clinical reconstruction algorithm did not include a background correction. Since SPECT-clinical reconstructions overestimated the LSF for high activities, and a background correction will only impact on low activity scans, this overestimation would probably not be corrected by including a background correction. However, switching to an MC-based reconstruction model, and thereby including scatter correction, does impact the LSF estimation at the full range of activities. Adding an additional background correction to this MC-based reconstruction model again only influences scans with a low activity. Therefore, for Bremsstrahlung SPECT/CT, proper modelling of scatter is more important than including a background correction for accurate LSF estimation. Only for the low activity scans ( $<100 \mathrm{MBq}$ ) a combination of MC-based modelling and background correction is necessary in order to acquire accurate LSF estimations. However, since a background correction seems to underestimate the LSF at low activities (as opposed to a tendency to overestimate the LSF without background correction) one may conservatively choose not to use a background correction.

For the PET acquisitions the background correction could not be incorporated in the clinical Siemens reconstruction algorithm, like is the case for SPECT-MC-BGcorr, and is therefore performed on an image basis. ${ }^{90} \mathrm{Y}$ PET suffers from low count rate and high random fraction. In addition, there is the natural background signal originating from the ${ }^{176} \mathrm{Lu}$ in the LSO crystal. Both this, and the resulting sparse sinograms, are prone to a positive bias in maximum likelihood reconstructions at low activities [156]. The algorithm used in this study was designed to minimize the effects of algorithm bias to the sparse data. Still, Carlier et al. show that increasing random fractions increase algorithm bias [149]. Their study reports small algorithm bias for random fractions ranging from 0.70 to 0.85. Random fractions in our study ranged from 0.91 to 0.99 , indicating we can expect considerably more algorithm bias than observed by Carlier et al.. By performing a long background measurement (24 hours), we attempted to obtain a reliable background estimation. Subtracting the reconstructed background activity from our reconstructed phantom activity did improve the LSF estimation, but still shows large overestimation of LSF for low activities, indicating that our PET reconstructions suffer more from the algorithm bias than from the background activity. Correcting this algorithm bias requires (partly) redesigning the reconstruction algorithm, which was beyond the scope of this research.

The activity recovery of the PET reconstructions showed good agreement with the study by Willowson et al. [148]. They reported good activity recovery for an activity concentration of $37 \mathrm{kBq} / \mathrm{mL}$. We found good activity recovery (less than 10\% difference) for activities as low as $45 \mathrm{MBq}$, which translates to an activity concentration of $41 \mathrm{kBq} / \mathrm{mL}$ for the liver compartment and $23 \mathrm{kBq} / \mathrm{mL}$ for the lung compartment. 
Willowson et al. [157] recommend slightly different reconstruction settings for our scanner (recommended: 2 iterations, 21 subsets; this study: 4 iterations, 21 subsets). For these recommended settings they show an average error in activity concentration at 300 $\mathrm{kBq} / \mathrm{mL}$ of $-2 \%$ (range $+4 \%$ to $-9 \%$ ). We show an error in activity concentration at $260 \mathrm{kBq} /$ $\mathrm{mL}$ of $-1 \%$. This error is within the range reported by Willowson et al..

Limitations of our study include that, owing to practical reasons, no phantom data with a 0\% LSF was acquired. Therefore, a 0\% LSF was simulated by delineating an additional, cold VOI. This simulation of a 0\% LSF was suboptimal because the cold VOI was a water compartment instead of a lung-like compartment. Furthermore, the cold $\mathrm{VOI}$ compartment in this phantom was located close to the liver. This position resulted in more spillover activity from the liver inside the cold VOI than in the top part of the lung VOI, which is located relatively far from the liver. The effect of the position of the cold VOI in relation to the liver can be observed by comparing the LSF estimations of the planar acquisitions for the 15\% and 0\% LSF simulations. LSF estimations by planar imaging were heavily influenced by the spillover activity from the liver and resulted in higher LSF estimations for the cold VOI (0\%) than for the lung VOI (15\%). It is expected that a $0 \%$ LSF measurement with no activity in the lungs in a clinical setting would result in less overestimation of the LSF as compared with the current method with the cold VOI.

Another limitation of our study is the fact that MC-based SPECT/CT reconstructions take more time (approximately 3 hours) than clinical reconstructions and that MC-based reconstruction algorithms are not available for clinical practice. For availability of the MCbased reconstruction algorithm used in this study, please contact the authors.

Our phantom was filled with ${ }^{90} \mathrm{Y}$ chloride and has uniform activity distribution throughout the liver and the lungs, whereas in a clinical setting, patients treated with ${ }^{90} Y$ microspheres might have non-uniform activity distribution within the liver and/or lungs. This could influence image quality and quantification and therefore affect LSF estimation.

Another issue that might be encountered in a clinical setting are motion artefacts. Breathing can cause a mismatch between CT and PET or SPECT, which can result in spillover of liver activity into the lungs. This spillover effect can result in an overestimation of the LSF.Yu et al. [40] show that this can be substantially avoided by excluding the region of the lungs that is within $2 \mathrm{~cm}$ of the diaphragm.

This study focused on the LSF estimation part of the ${ }^{90} Y$ pre-treatment procedure. It should be regarded as a first step toward application of a ${ }^{90} \mathrm{Y}$ pre-treatment procedure. A next step toward implementation could be a multicenter phantom study to verify repeatability, or a clinical patient study including a ${ }^{90} Y$ pre-treatment procedure in combination with a ${ }^{99 m} \mathrm{TC}$-MAA pre-treatment procedure for comparison. This would require the ordering of a small dosage of ${ }^{90}$, but it is not uncommon to order multiple vials (one of which could be used for the pre-treatment procedure) summing to an overall desired activity, as was done in same-day radioembolization procedures [158].

\section{Conclusions}

This study evaluated the ability to accurately estimate the LSF of ${ }^{90}$ Y-based radioembolization by means of a low activity ${ }^{90} \mathrm{Y}$-based pre-treatment procedure. The imaging methods investigated comprised PET/CT, Bremsstrahlung SPECT/CT, and planar imaging. Bremsstrahlung SPECT/CT reconstructed with an MC-based model including a background correction can accurately estimate the LSF for a ${ }^{90} Y$ pre-treatment procedure using a theoretically safe ${ }^{90} \mathrm{Y}$ activity as low as $70 \mathrm{MBq}$. 



\section{Abstract}

Purpose: Prior to ${ }^{90} \mathrm{Y}$ hepatic radioembolization, a dosage of ${ }^{99 \mathrm{~m} T \mathrm{C}-m a c r o a g g r e g a t e d}$

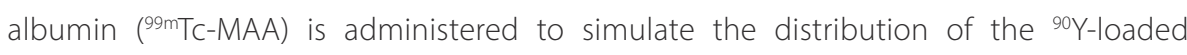
microspheres. This pre-treatment procedure enables lung shunt estimation, detection of potential extrahepatic depositions, and estimation of the intrahepatic dose distribution. However, the predictive accuracy of the MAA particle distribution is often limited. Ideally, ${ }^{90} \mathrm{Y}$ microspheres would also be used for the pre-treatment procedure. Based on previous research, the pre-treatment activity should be limited to the estimated safety threshold of $100 \mathrm{MBq}$, making imaging challenging. The purpose of this study was to evaluate the quality of intra- and extrahepatic imaging of ${ }^{\circ 0}$ - -based pre-treatment PET/CT and quantitative SPECT/CT scans, by means of phantom experiments and a patient study.

Methods: An anthropomorphic phantom with three extrahepatic depositions was filled with ${ }^{90} Y$ chloride to simulate a lung shunt fraction (LSF) of 5.3\% and a tumour to nontumour ratio (T/N) of 7.9. PET/CT (Siemens Biograph $\mathrm{mCT}$ ) and Bremsstrahlung SPECT/ CT (Siemens Symbia T16) images were acquired at activities ranging from 1999 MBq down to $24 \mathrm{MBq}$, representing post- and pre-treatment activities. PET/CT images were reconstructed with the clinical protocol and SPECT/CT images were reconstructed with a quantitative Monte Carlo-based reconstruction protocol. Estimated LSF, T/N, contrast to noise ratio of all extrahepatic depositions, and liver parenchymal and tumour dose were compared with the phantom ground truth. A clinically reconstructed SPECT/CT of 150 $\mathrm{MBq}{ }^{99 \mathrm{mT}} \mathrm{Tc}$ represented the current clinical standard. In addition, a ${ }^{90} \mathrm{Y}$ pre-treatment scan was simulated for a patient by acquiring post-treatment PET/CT and SPECT/CT data with shortened acquisition times.

Results: At an activity of $100 \mathrm{MBq}{ }^{90} \mathrm{Y}, \mathrm{PET} / \mathrm{CT}$ overestimated LSF (+10 percentage point $(\mathrm{pp})$ ), underestimated liver parenchymal dose (-3 Gy/GBq), and could not detect the extrahepatic depositions. SPECT/CT more accurately estimated LSF (-0.7 pp), parenchymal dose (-0.3 Gy/GBq) and could detect all three extrahepatic depositions. ${ }^{99 m}$ TC SPECT/CT showed similar accuracy as ${ }^{90} \mathrm{Y}$ SPECT/CT (LSF: +0.2 pp, parenchymal dose: +0.4 Gy/GBq all extrahepatic depositions visible), although the noise level in the liver compartment was considerably lower for ${ }^{99 \mathrm{~m} T C}$ SPECT/CT compared to ${ }^{90} \mathrm{Y} \mathrm{SPECT/CT.} \mathrm{The} \mathrm{patient's} \mathrm{SPECT/CT}$ simulating a pre-treatment ${ }^{90} \mathrm{Y}$ procedure accurately represented the post-treatment ${ }^{90} \mathrm{Y}$ microsphere distribution

Conclusion: Quantitative SPECT/CT of $100 \mathrm{MBq}{ }^{90} \mathrm{Y}$ could accurately estimate LSF, T/N parenchymal and tumour dose, and visualise extrahepatic depositions.

\section{Introduction}

Prior to ${ }^{90} \mathrm{Y}$ radioembolization treatment of liver tumours, a dosage of ${ }^{99 \mathrm{mT} \mathrm{T} \text { - }}$ macroaggregated albumin ( $\left.{ }^{99 m} \mathrm{Tc}-\mathrm{MAA}\right)$ is administered to simulate the distribution of the ${ }^{90}$-loaded microspheres. This pre-treatment safety procedure is mainly performed to estimate the lung shunt fraction (LSF) and to detect potential extrahepatic depositions [37]. In addition, a single photon emission computed tomography/computed tomography (SPECT/CT) scan of ${ }^{99 m} \mathrm{TC}-M A A$ may be used for intrahepatic dosimetry [122].

The prescribed activity of ${ }^{90} Y$ microspheres can be calculated based on the Body Surface Area (BSA)-method for resin microspheres or the mono-compartment model for glass microspheres $[25,127]$. Both methods assume a uniform dose distribution within the (targeted) liver volume, and thereby neglect individual patient's dose distribution. Another model to calculate prescribed activity is the partition model, which takes the average tumour to non-tumour ratio into account [159]. However, this method may still be an oversimplification in the case of multiple tumours with varying uptake.[122] As an alternative to the tumour to non-tumour ratio, the tumour dose may be maximized based on the maximal parenchymal dose [123].

All image-based dosimetry methods rely on the accuracy of the pre-treatment procedure,

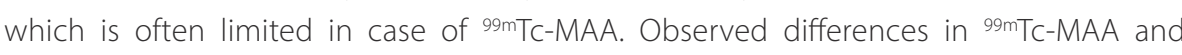
${ }^{90} Y$-microsphere distributions could be caused by their differences in shape and size and by free circulating pertechnetate $[34,42,44]$. A better representation of the microsphere distribution may be achieved by using identical particles for pre-treatment and treatment, as is feasible in ${ }^{166} \mathrm{Ho}$ microsphere radioembolization $[144,145]$

A similar objective may be pursued for ${ }^{90} Y$-loaded microspheres. However, the total energy absorbed per $\mathrm{Bq}$ is higher for ${ }^{90} \mathrm{Y}$ than for ${ }^{166} \mathrm{Ho}$, which limits the pre-treatment activity to the estimated safety threshold of $100 \mathrm{MBq}[29,140,145]$. In addition, SPECT of ${ }^{90} Y$ is based on Bremsstrahlung imaging, which prevents the use of a photopeak energy window and energy window based scatter correction. Quantitative SPECT can be accomplished by applying Monte Carlo based reconstruction [17]. Alternatively, ${ }^{90} \mathrm{Y}$ may be imaged using positron emission tomography (PET). However, the small positron branching ratio of ${ }^{90} \mathrm{Y}$ in combination with the required low activity for pre-treatment makes PET challenging.

In an earlier study, we demonstrated the feasibility of accurately estimating the LSF of a low activity ${ }^{90} Y$-based pre-treatment procedure when imaged with SPECT/CT and reconstructed with a Monte Carlo-based reconstruction model to include scatter correction [140]. In the present study it was investigated whether low activity ${ }^{90}$ Y SPECT/ 
CT and PET/CT allowed detection of extrahepatic depositions and intrahepatic dosimetry, using an anthropomorphic phantom and short acquisition scans of a clinical patient to simulate ${ }^{90} Y$ pre-treatment scan.

\section{Methods}

\section{Phantom}

We used an anthropomorphic thorax phantom (model ECT/TOR/P, IEL, Chilcompton, UK) with a liver compartment, lung compartments (filled with styrofoam beads), and a spine insert. Two tumour compartments were added to the liver (one solid and one necrotic tumour) and three extrahepatic depositions were added to the background volume. The extrahepatic depositions were located posteriorly to the liver and below the left lung (small extrahepatic deposition), posteriorly to the liver and below the right lung (medium sized extrahepatic deposition) and above the liver and in between the lungs (large extrahepatic deposition) (Figure 6.1).

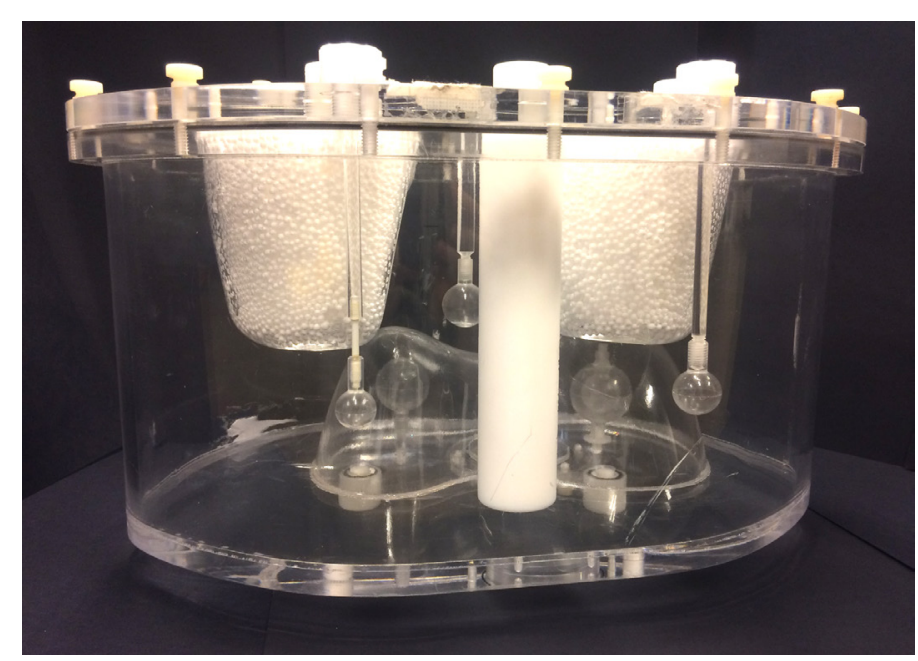

Figure 6.1: Anthropomorphic phantom including liver, lung, tumour, and extrahepatic deposition compartments.

The phantom was filled with $2.0 \mathrm{GBq}{ }^{90} \mathrm{Y}$ chloride in $0.5 \mathrm{M}$ of $\mathrm{HCl}$ to prevent adhesion to the plastic phantom walls [153]. The initial activity, size, and shape of all compartments are listed in Table 6.1. The phantom had a lung shunt fraction (LSF) of 5.3\% and a tumour to non-tumour ratio ( $\mathrm{T} / \mathrm{N})$ of 7.9. Based on the results of a previous study by Prince et al., the activity concentration of the extrahepatic depositions was chosen to be $1.3 \%$ of the total activity in the phantom divided by $6.8 \mathrm{~mL}$ [145].

\begin{tabular}{lccc}
\multicolumn{4}{l}{ Table 6.1: Initial activity, size, and shape of the compartments within the anthropomorphic phantom. } \\
\hline & $\begin{array}{c}\text { Initial activity } \\
\text { [MBq] }\end{array}$ & Size $[\mathbf{m L}$ ] & Shape \\
\hline Liver & 1477 & $1.2 \cdot 10^{3}$ & Liver \\
Lungs & 102 & $2.1 \cdot 10^{3}$ & Left and right lung \\
Solid intrahepatic tumour & 158 & 15.9 & Sphere \\
Necrotic intrahepatic tumour & 188 (outer rim), & 18.9 (outer rim), & Sphere inside \\
& 0 (core) & 5.6 (core) & a sphere \\
Small extrahepatic deposition & 7 & 2.0 & Sphere \\
Medium extrahepatic deposition & 15 & 4.2 & Sphere \\
Large extrahepatic deposition & 30 & 8.2 & Sphere \\
\hline
\end{tabular}

To represent the current clinical pre-treatment procedure using ${ }^{99 m} \mathrm{TC}-\mathrm{MAA}$, the phantom

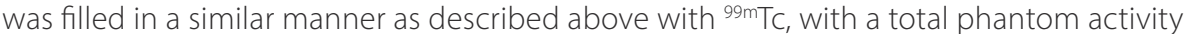
of $153 \mathrm{MBq}$, an LSF of $5.2 \%$, and a T/N of 7.6

\section{Image acquisition}

Thirteen PET/CT and SPECT/CT scans were acquired of the anthropomorphic phantom as the ${ }^{90} \mathrm{Y}$ activity decayed from $1999 \mathrm{MBq}$ down to $24 \mathrm{MBq}$. The total activity of the phantom at the time of imaging is listed in Table 6.2. For all scans, a CT scan was made for attenuation correction and to support delineation.

Table 6.2: Total activity of anthropomorphic phantom at time of imaging

\begin{tabular}{lccccccccccccc}
\hline Scan number & $\mathbf{1}$ & $\mathbf{2}$ & $\mathbf{3}$ & $\mathbf{4}$ & $\mathbf{5}$ & $\mathbf{6}$ & $\mathbf{7}$ & $\mathbf{8}$ & $\mathbf{9}$ & $\mathbf{1 0}$ & $\mathbf{1 1}$ & $\mathbf{1 2}$ & $\mathbf{1 3}$ \\
\hline $\begin{array}{l}\text { Total activity PET } \\
\text { (MBq) }\end{array}$ & 1966 & 1178 & 701 & 438 & 248 & 149 & 114 & 95 & 73 & 53 & 40 & 31 & 24 \\
$\begin{array}{l}\text { Total activity SPECT } \\
\text { (MBq) }\end{array}$ & 1999 & 1192 & 727 & 441 & 251 & 148 & 116 & 96 & 74 & 52 & 41 & 31 & 24 \\
\hline
\end{tabular}

All ${ }^{90} \mathrm{Y}$ PET/CT images were acquired on a Siemens Biograph $\mathrm{mCT}$ time of flight (TOF) scanner. Two bed positions were scanned to fit the entire phantom in the field of view. Acquisition time was 15 minutes per bed position, resulting in a total acquisition time of 30 minutes. Consecutive bed positions overlapped approximately $43 \%$.

All ${ }^{90} Y$ SPECT/CT images were acquired on a dual-head Siemens Symbia T16 scanner. Photons were acquired in a 50-250 keV energy window with the high-energy collimators mounted. Projections were acquired for 30 seconds per angle, using 120 angles over 360 degrees, resulting in a total acquisition time of 30 minutes. 
The ${ }^{99 m}$ TC SPECT/CT image was acquired on a dual-head Siemens Symbia T16 scanner. Photons were acquired in a 129-150 keV photopeak window and a 108-129 keV lower scatter window with the low-energy high-resolution collimators mounted. Projections were acquired for 20 seconds per angle, using 120 angles over 360 degrees, resulting in a total acquisition time of 20 minutes.

For ${ }^{90}$ Y SPECT a single background measurement was performed without the phantom present using the settings described above. For ${ }^{90} \mathrm{Y}$ PET, a single long background measurement (total scan time of 24 hours) was performed with the phantom without activity in the scanner. These background measurements were used to perform background corrections.

\section{Image reconstruction}

${ }^{90}$ Y PET projections were reconstructed with an Ordinary Poisson Ordered Subset Expectation Maximization (OP-OSEM) reconstruction algorithm, including resolution recovery (TrueX), TOF information, random, attenuation, scatter, dead time, and decay correction. The reconstruction used 4 iterations with 21 subsets, and a $5 \mathrm{~mm}$ full width at half maximum (FWHM) Gaussian post-reconstruction filter was applied. The reconstructed voxel size was $4.1 \times 4.1 \times 3.0 \mathrm{~mm}^{3}$. The background measurement was reconstructed using the same settings. Background correction was performed by subtraction (for mean or summed values) or quadratic addition (for standard deviations) of the reconstructed activity in the volumes of interest (VOIs) of the background reconstruction, from the reconstructed activity in the VOIs of the ${ }^{90} \mathrm{Y}$ reconstruction.

${ }^{90} Y$ SPECT projections were reconstructed with a Monte Carlo (MC) based OSEM reconstruction algorithm, including attenuation correction, resolution recovery, and MCbased scatter correction. The background correction was performed by adding the average measured background count to the forward projection. Since MC-based reconstructions take longer to converge, the reconstruction used 60 iterations with 8 subsets, and an 8 $\mathrm{mm}$ FWHM Gaussian post-reconstruction filter was applied [17]. The reconstructed voxel size was $4.8 \times 4.8 \times 4.8 \mathrm{~mm}^{3}$

99mTC SPECT projections were reconstructed using an OSEM reconstruction algorithm with flash3D, dual energy window scatter correction, and attenuation correction. The reconstruction used 6 iterations with 8 subsets, and a $5 \mathrm{~mm}$ FWHM Gaussian postreconstruction filter was applied. The reconstructed voxel size was $2.4 \times 2.4 \times 2.4 \mathrm{~mm}^{3}$.

\section{Analysis}

The liver, lungs, tumours, and extrahepatic depositions were semi-automatically delineated on one CT scan using 3D Slicer [130]. The reference CT was rigidly registered to all other CT scans, and all VOIs were transformed accordingly, to ensure that the same volume was analysed for each scan. Registrations and transformations were performed using Elastix and were visually inspected [129]. The liver, lungs, and extrahepatic deposition VOls were dilated by the spatial resolution of each system ( $14 \mathrm{~mm}$ for ${ }^{90} \mathrm{Y}$ SPECT and $6 \mathrm{~mm}$ for ${ }^{90} \mathrm{Y}$ PET and ${ }^{99 \mathrm{~m} T C}$ SPECT) to partially compensate for the partial volume effect. For ${ }^{90} \mathrm{Y}$ SPECT the dilation resulted in overlapping lung and liver VOIs, and the liver VOI was chosen to have priority over the lung VOI (as is custom in clinical practice).

\section{Mono-compartment model}

The mono-compartment model uses the LSF to calculate the prescribed activity for radioembolization. The LSF was calculated as:

$$
\mathrm{LSF}=\frac{\mathrm{C}_{\text {lung }}}{\mathrm{C}_{\text {lung }}+\mathrm{C}_{\text {liver }}} \cdot 100 \%
$$

where $C_{\text {lung }}$ is the total number of counts in the dilated lung VOI and $C_{\text {liver }}$ is the total number of counts in the dilated liver VOI.

In addition, potential extrahepatic depositions need to be detected in a pre-treatment scan. As a measure for detectability, the contrast to noise ratio (CNR) for each extrahepatic deposition was calculated as:

\section{$\mathrm{CNR}=\frac{\overline{\text { extrahepatic_deposition }}-\overline{\text { background }}}{\mathrm{S}_{\text {backgound }}}$}

where extrahepatic_deposition is the mean pixel value of the non-dilated extrahepatic deposition VOI, background is the mean pixel value of the background VOI (defined as the entire phantom minus the dilated VOIs of the liver, lungs and extrahepatic depositions), and $\mathrm{S}_{\text {background }}$ is the standard deviation of the background VOI.

The low count rate, and the ensuing high noise level, may induce false positive detection of extrahepatic depositions. To identify the presence of such false positives, spherical VOls with the same size as the extrahepatic depositions were centered on all voxels within the phantom background VOI, and the associated CNR of these background spheres was calculated using equation 2, where the background is defined as the background VOI minus the background sphere. An extrahepatic deposition was considered to be detectable when its CNR was twice that of the largest CNR of the background spheres. 


\section{Multi compartment model}

Another way to calculate the prescribed activity for radioembolization is by using the partition model, which in addition to the LSF, uses the T/N. To calculate the T/N, a parenchymal VOI was created by subtracting the tumour VOIs from the liver VOI. T/N was calculated as:

\section{$\mathrm{T} / \mathrm{N}=\frac{\overline{\text { tumour }}}{\overline{\text { parenchyma }}}$}

where $\overline{\text { tumour }}$ is the mean pixel value of the non-dilated tumour VOI and $\overline{\text { parenchyma }}$ is the mean pixel value of the non-dilated parenchymal VOI.

The prescribed activity for radioembolization can also be based on a threshold for parenchymal dose and/or tumour dose. The predicted dose per GBq injected ${ }^{9} Y$ was calculated as:

$$
\mathrm{D}_{\text {target }}=\frac{\frac{\mathrm{C}_{\text {target }}}{\mathrm{C}_{\text {total }}} \cdot 50}{\mathrm{~m}_{\text {target }}}
$$

where $C_{\text {target }}$ is the total number of counts in the non-dilated target VOI. The target is either the parenchyma, solid tumour or necrotic tumour. Fifty is the absorbed energy in joule from the decay of $1 \mathrm{GBq}$ of ${ }^{90} \mathrm{Y}$, and $\mathrm{m}_{\text {target }}$ is the mass of the target $\mathrm{VOI}$ in $\mathrm{kg}$ (determined using the target $\mathrm{VOI}$ and a conversion factor of $1.03 \mathrm{~g} / \mathrm{mL}$ ) [29]. $C_{\text {total }}$ is the total number of reconstructed counts and was defined as:

\section{$\mathrm{C}_{\text {total }}=\mathrm{C}_{\text {liver }}+\mathrm{C}_{\text {lung }}+\mathrm{C}_{\text {extrahepatic depositions }}$}

This definition of $C_{\text {total }}$ is based on the assumption that all injected activity ends up in either the liver or the lungs, and that for this particular phantom there is also activity located in the extrahepatic depositions.

The SPECT reconstructions were calibrated against an external source to yield $\mathrm{Bq} / \mathrm{mL}$ by scaling the total number of reconstructed counts to the injected activity. The PET reconstructions already provide $\mathrm{Bq} / \mathrm{mL}$ and thus it was possible to directly calculate the dose per GBq injected ${ }^{90} \mathrm{Y}$ as:

$\mathrm{D}_{\text {target }}\left(\right.$ PET only) $=\frac{\frac{A_{\text {target }}}{A_{\text {total }}} \cdot 50}{m_{\text {target }}}$

where $A_{\text {target }}$ is the activity in the non-dilated target VOI in GBq and $A_{\text {total }}$ is the known total activity in the phantom in GBq.

\section{Voxel-based dosimetry}

Two additional metrics to those that could be used for the calculation of prescribed activity were analysed. These are the CNR of the tumours, calculated according to equation 2 where the background is the parenchyma VOI eroded by the spatial resolution of each system, and the background variability (BV) of the parenchyma calculated as:

\section{$\mathrm{BV}=\frac{S_{\text {parenchyma }}}{\overline{\text { parenchyma }}}$}

where $\mathrm{S}_{\text {parenchyma }}$ is the standard deviation of the parenchyma VOI eroded by the spatial resolution of each system and $\overline{\text { parenchyma }}$ is the mean pixel value of the parenchyma VOI eroded by the spatial resolution of each system.

\section{Patient dosimetry}

To evaluate the image quality for a more inhomogeneous activity distribution, one patient who underwent radioembolization with ${ }^{90} Y$-doped glass microspheres (TheraSphere, BTG International Ltd.) gave informed consent to receive additional scans. The patient received a total of $3.87 \mathrm{GBq}{ }^{90} \mathrm{Y}(3.12 \mathrm{GBq}$ at time of PET imaging and $3.09 \mathrm{GBq}$ at time of SPECT imaging). At our institute, all radioembolization patients receive a post-treatment PET/CT with the image acquisition and reconstruction settings described in the previous section.

This patient received an additional PET with shortened acquisition time of 29 seconds per bed position (instead of 15 minutes per bed position) to simulate a pre-treatment scan of $100 \mathrm{MBq}{ }^{90} \mathrm{Y}$. In addition, this patient received a SPECT/CT with the image acquisition and reconstruction settings described in the previous section, and a SPECT with shortened acquisition time of 1 second per view (instead of 30 seconds per view) to simulate a pretreatment scan of $100 \mathrm{MBq}{ }^{90} \mathrm{Y}$. A SPECT background measurement was performed with an acquisition time of 29 seconds per view. These background projections were added to the $1 \mathrm{sec} / v i e w$ projections to ensure the right noise level. Prior to performing the clinical scan, we tested whether a short scan accurately mimics a low activity scan by acquiring additional short acquisitions of the anthropomorphic phantom at 1984, 1182 and $711 \mathrm{MBq}$, and comparing the metrics described above with the anthropomorphic scan acquired at $100 \mathrm{MBq}$

Liver VOIs were manually drawn on the low dose CTs and were split into two VOls: a highdose (HD) and a low-dose (LD) VOI. The HD VOI included all voxels with a concentration greater than twice the average liver activity concentration on one of the reconstructed images; the LD VOI included all remaining liver voxels. The SPECT and PET reconstructed counts were converted into units of activity by normalisation of the total counts in the liver VOI to the activity administered to the patient. The absorbed dose images assumed 
that all emitted energy was absorbed within the voxel of origin. Mean absorbed dose and cumulative dose volume histograms were calculated for both VOls.

\section{Results}

\section{Phantom study}

Figure 6.2 shows axial and coronal views of the PET and SPECT reconstructions of the phantom. At 2.0 GBq, both ${ }^{90} \mathrm{Y} \mathrm{PET} \mathrm{and}{ }^{90} \mathrm{Y}$ SPECT clearly visualised the solid and necrotic tumour, as well as all extrahepatic depositions. The PET reconstruction had a higher spatial resolution than the SPECT reconstruction, which led to a more apparent 'cold' core of the necrotic tumour. At $\sim 100 \mathrm{MBq}$, noise started to prevail in the PET reconstruction and the extrahepatic depositions were not visible anymore. However, for the SPECT reconstruction at $\sim 100 \mathrm{MBq}$, all extrahepatic depositions were still visible. For ${ }^{99 \mathrm{~m} T C}$ SPECT, both tumours and the extrahepatic depositions were clearly visible.

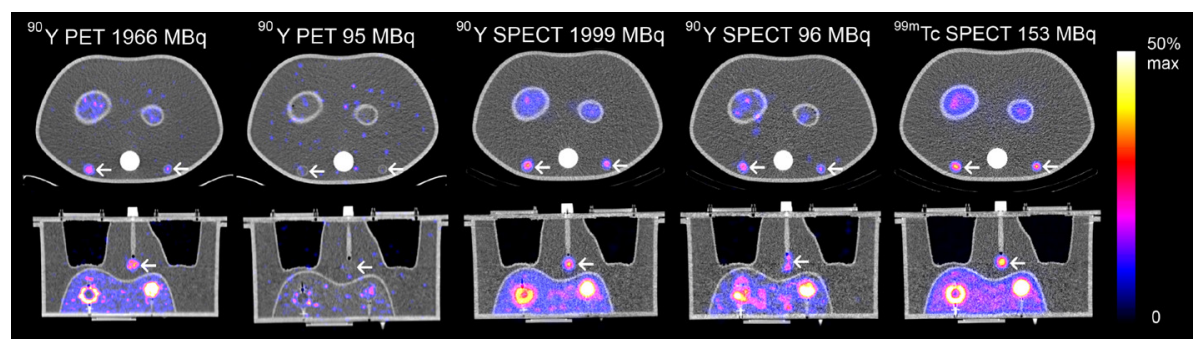

Figure 6.2: Axial (top row) and coronal (bottom row) views of the phantom with $x$-ray images in gray scale and nuclear images in colour overlay, scaled between 0 and $50 \%$ of the maximum number of counts in the reconstruction. Arrows indicate the locations of the extrahepatic depositions.

\section{Mono-compartment model}

Figure 6.3A shows the LSF as a function of phantom activity. PET accurately estimated the LSF (absolute difference $<1$ percent point (pp)) for activities over $\sim 1 \mathrm{GBq}$, and overestimated the LSF for activities below $\sim 1 \mathrm{GBq}$. SPECT accurately estimated the LSF (absolute difference $<1 \mathrm{pp}$ ) for activities over $\sim 50 \mathrm{MBq}$. The ${ }^{99 \mathrm{~m} T C}$ SPECT estimated an LSF of $5.0 \%$ (where the true LSF was 5.2\%).

Figure 6.3B shows the CNR for the extrahepatic depositions within the phantom. For both PET and SPECT the CNR of the extrahepatic depositions decreased with the phantom activity. SPECT had a higher CNR than PET, over the entire range of phantom activities. The CNR of the ${ }^{99 \mathrm{~m} T C}$ SPECT was slightly higher than the CNR of ${ }^{90} \mathrm{Y} \mathrm{SPECT}$ at $~ 100 \mathrm{MBq}$. Table 6.3 shows the minimal total activity at which the extrahepatic depositions were detectable. The smaller the extrahepatic deposition, the larger the minimal total activity needed. For SPECT, all extrahepatic depositions were detectable at the activity that would theoretically be safe to use for a ${ }^{90} \mathrm{Y}$-based pre-treatment scan (100 MBq).
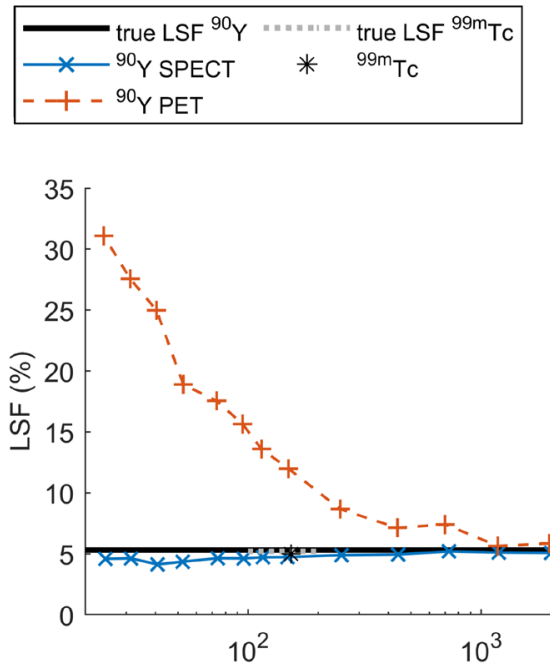

total activity phantom $(\mathrm{MBQ})$
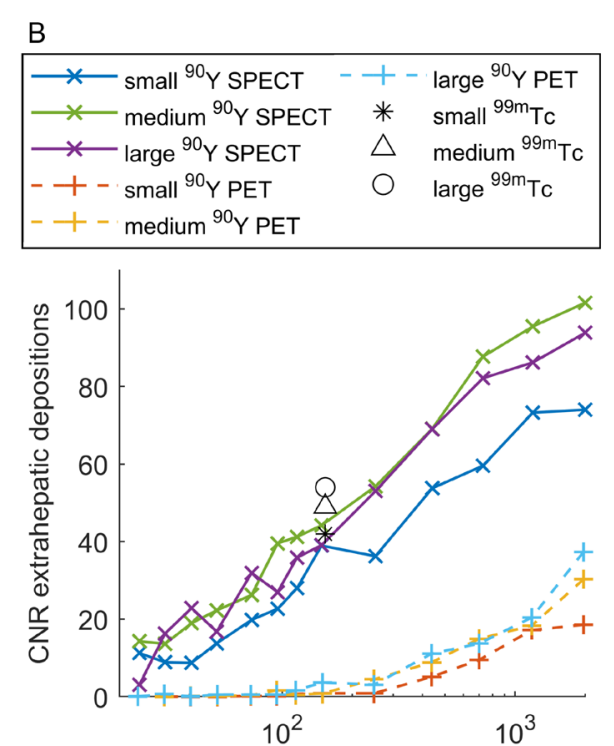

total activity phantom $(\mathrm{MBq})$
Figure 6.3: A) LSF as a function of total phantom activity. B) CNR of the small, medium and large extrahepatic depositions as a function of total phantom activity.

Table 6.3: Total activity in the phantom (and activity concentration of the extrahepatic depositions between parentheses) at which extrahepatic depositions were detectable.

\begin{tabular}{lccc}
\hline \multicolumn{1}{c}{ Large deposition } & Medium deposition & Small deposition \\
\hline $\mathrm{PET}$ & $>\sim 300 \mathrm{MBq}(0.6 \mathrm{MBq} / \mathrm{mL})$ & $>\sim 300 \mathrm{MBq}(0.6 \mathrm{MBq} / \mathrm{mL})$ & $>\sim 1000 \mathrm{MBq}(1.9 \mathrm{MBq} / \mathrm{mL})$ \\
$\mathrm{SPECT}$ & $>\sim 30 \mathrm{MBq}(0.06 \mathrm{MBg} / \mathrm{mL})$ & $>\sim 45 \mathrm{MBq}(0.08 \mathrm{MBq} / \mathrm{mL})$ & $>\sim 90 \mathrm{MBq}(0.2 \mathrm{MBq} / \mathrm{mL})$ \\
\hline
\end{tabular}

\section{Multi compartment model}

Figure 6.4A shows the estimated parenchymal dose per GBq of ${ }^{90} \mathrm{Y}$ injected. For SPECT, the estimated parenchymal dose was accurate (absolute difference $<1$ Gy/GBq) over the entire range of ${ }^{90} \mathrm{Y}$ activities. For PET, scaling the reconstructed counts to the injected activity resulted in accurate parenchymal dose estimates (absolute difference <1 Gy/GBq) for activities over 1 GBq. Calculating the dose using the quantitative nature of PET led to underestimation of the dose for activities over $\sim 70 \mathrm{MBq}$ and to overestimation of the dose for activities under $\sim 70 \mathrm{MBq}$. The ${ }^{99 \mathrm{mTC}}$ SPECT estimated parenchymal dose was $31.1 \mathrm{~Gy} /$ $\mathrm{GBq}$ (where the true dose was $31.3 \mathrm{~Gy} / \mathrm{GBq}$ ). 


\section{Patient dosimetry}

The method of adding a background measurement to the measured sinogram to achieve the right noise level for a simulated low activity scan for SPECT was tested with the anthropomorphic phantom. It showed that the simulated low activity scans acquired at a true total activity of 1984, 1182 and $711 \mathrm{MBq}$ resemble a measured low activity scan of 100 MBq quite well in terms of LSF (difference less than $0.1 \mathrm{pp}$ ), parenchymal dose (difference less than $0.5 \mathrm{~Gy} / \mathrm{GBq}$ ) and tumour dose (difference less than $20 \mathrm{~Gy} / \mathrm{GBq}$ ). We therefore conclude that the simulated $100 \mathrm{MBq}$ scan of the patient was a good indicator for the image quality of a ${ }^{90}$-based pre-treatment scan.

Figure 6.6 shows the dose volume histograms and the axial slices of the dose maps of the SPECT scan of the patient at long and short acquisitions times. Visually, the dose maps were very similar. Quantitatively, the dose volume histograms were similar and the mean dose for the LD and HD VOls were in close agreement (mean doses HD: 497 and 518 Gy, mean doses LD: 32.5 and 28.1 Gy, D50\% HD: 405 and 407 Gy, D50\% LD: 3.6 and $1.8 \mathrm{~Gy}$, for long and short acquisition times respectively).
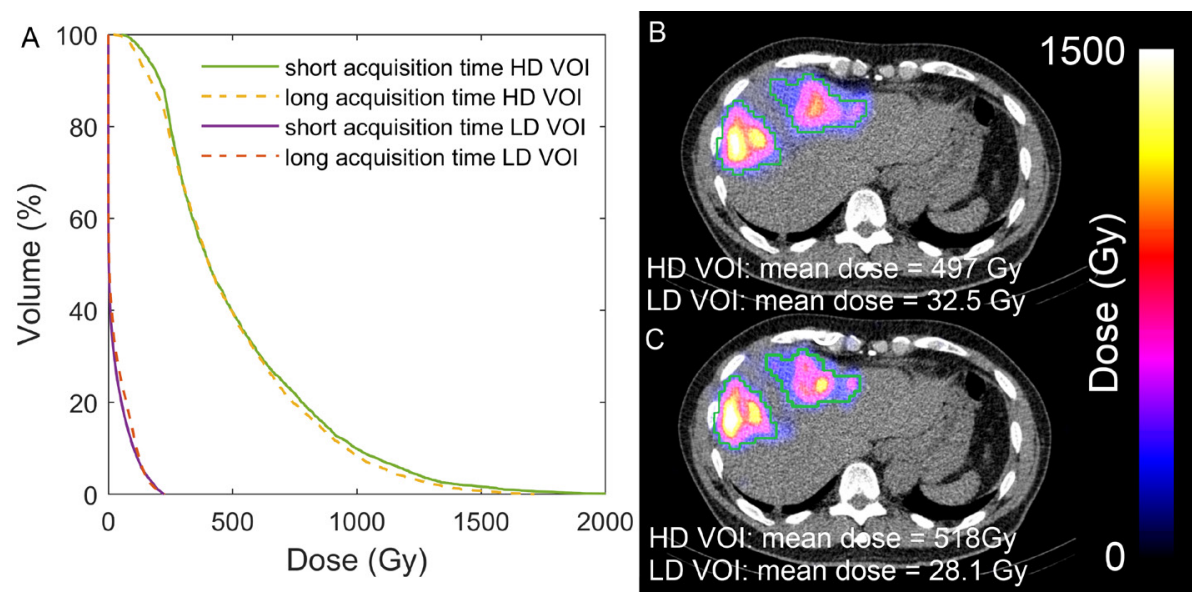

Figure 6.6: Cumulative dose volume histograms of the SPECT/CT scans with long and short acquisition times (A). Axial slice of the dose map of the long acquisition time (B) and of the short acquisition time (C). The green delineation represents the high dose (HD) VOI. The low dose (LD) VOI consists of the remaining liver.

Figure 6.7 shows the dose volume histograms and the axial slices of the dose maps of the PET scan of the patient at long and short acquisition times. Visually, the short acquisition reconstruction was a lot noisier than the long acquisition reconstruction. Although the mean doses for the LD and HD VOls were relatively comparable, the dose volume histograms showed differences, implying substantial differences in dose distribution (mean doses HD: 608 and 663 Gy, mean doses LD: 16.0 and 6.2 Gy, D50\% HD: 482 and 328 Gy, D50\% LD: 2.0 and 0.0 Gy, for long and short acquisition times respectively).
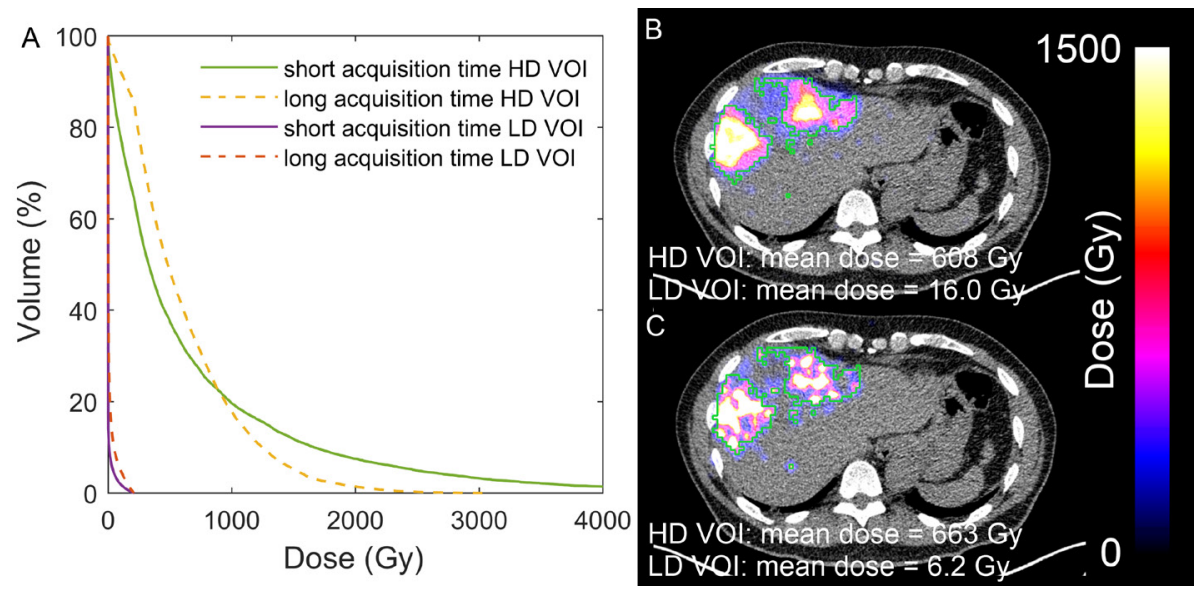

Figure 6.7: Cumulative dose volume histograms of the PET/CT scan with long and short acquisition times (A). Axial slice of the dose map of the long acquisition time (B) and of the short acquisition time (C). The green delineation represents the high dose (HD) VOI. The low dose (LD) VOI consists of the remaining liver:

\section{Discussion}

This study showed that the image quality of a ${ }^{90} \mathrm{Y}$ pre-treatment SPECT/CT scan (100 $\mathrm{MBq}$ ), when reconstructed with an MC-based reconstruction model, was sufficiently accurate for LSF, T/N, parenchymal, and tumour dose estimation and that extrahepatic depositions could be detected. A simulated ${ }^{90} Y$ pre-treatment (100 MBq) SPECT/CT scan of a radioembolization patient showed accurate dosimetry. Furthermore, our previous results on accurate LSF estimation with ${ }^{90} Y$ SPECT/CT were confirmed [140]

The estimates of T/N and tumour dose were lower than the true T/N and tumour dose for both PET and SPECT, even at high phantom activities. This is caused by the partial volume effect. PET has a higher spatial resolution than SPECT and therefore the partial volume effect was more severe in SPECT than in PET (at 2.0 GBq, PET had a higher estimated T/N and tumour dose than SPECT). The partial volume effect also explains the higher estimated $\mathrm{T} / \mathrm{N}$ and tumour dose of the solid tumour as compared with the necrotic tumour, since the solid tumour had a smaller surface-volume ratio than the necrotic tumour. The increase in underestimation of T/N and tumour dose at low phantom activities for PET is caused by the dominant noise component in these images (at $24 \mathrm{MBq}, 61 \%$ of the trues could be attributed to background activity). 
To determine the detectability of extrahepatic depositions in a scan, ideally an observer study should be performed. Since this is not possible for a phantom study, we chose to compare the CNR of the extrahepatic depositions with the CNR of spheres placed in the cold background of the phantom, to study false positive detection. As a threshold value for detectability we chose a factor of two for the ratio between those CNRs in order to avoid background spheres that have similar visibility as the extrahepatic depositions.

The clinical standard for the pre-treatment procedure is the use of ${ }^{99 m} \mathrm{mc}-\mathrm{MAA}$. ${ }^{99 \mathrm{mTC}}$ with its single photopeak, is easier to image than ${ }^{90} Y$, which has a broad Bremsstrahlung

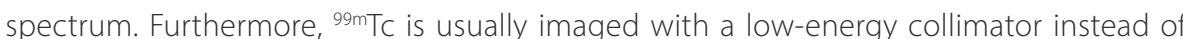

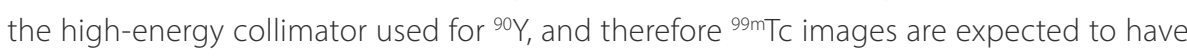
a better spatial resolution. It is thus expected that a pre-treatment ${ }^{99 m}$ TC-MAA SPECT will have a higher image quality than a pre-treatment ${ }^{90} Y$ SPECT. Even though image quality of pre-treatment ${ }^{99 m}$ TC-MAA SPECT is superior, as followed from the CNR for the extrahepatic depositions, CNR of the tumours, and background variability of the parenchyma, the quantitative measures LSF, T/N, parenchymal, and tumour dose were comparable for both ${ }^{99 m} \mathrm{Tc}$ and ${ }^{90} \mathrm{Y}$ pre-treatment scans.

Current guidelines on dosage calculation from the manufacturers of microspheres are all based on a mono-compartment model and advise to determine the lung shunt fraction before proceeding with radioembolization treatment $[24,25,127]$. This makes the LSF the most significant metric of this study for current clinical practice. A different approach to dosage calculation is to use a multi-compartment model, which would be more personalised. This requires a T/N ratio (partition model) for dosage calculation. The downside of using the $\mathrm{T} / \mathrm{N}$ ratio is that there is currently no consensus on how to calculate the T/N and how to deal with multiple tumours [122]. Chiesa et al. proposed to use a multi-compartment model using the parenchymal dose as input for dosage calculation [123]. Both LSF and parenchymal dose are metrics that could be accurately estimated by ${ }^{90} Y$ SPECT in this study. When moving toward voxel level estimates, a metric like the background variability (BV) becomes important. The BV favours the ${ }^{99 m} T C$ SPECT, which has a considerably lower BV than ${ }^{90} \mathrm{Y}$ SPECT. However, the dose volume histograms of the single patient show promising results, which might imply that voxel level estimates could be achievable for diagnostic activity ${ }^{90}$ Y SPECT.

From a biological point of view, it is expected that using ${ }^{90} \mathrm{Y}$ microspheres instead of ${ }^{99 \mathrm{mT}} \mathrm{T}$ MAA for the pre-treatment procedure will lead to a distribution that is more similar to the treatment procedure, since the same particle is used for the pre-treatment and treatment procedures. In combination with accurate imaging, this can lead to more accurate dose planning. However, since microspheres are not metabolised [109], the microspheres administered during a pre-treatment procedure will still be present in the liver vasculature during the treatment procedure, which could change the vasculature and limit the prognostic value of the pre-treatment procedure. It may be important to not only limit the activity of the pre-treatment procedure to avoid unintended radiation damage, but also to limit the number of microspheres administered to avoid an embolic effect. The ideal number of microspheres that should be used for a pre-treatment procedure needs further investigation.

A limitation of our study is that, owing to practical reasons, only one phantom configuration was scanned. The extrahepatic depositions all had the same activity concentration, and only three depositions were inserted in our phantom. The volumes of our extrahepatic depositions covered the lower end of the range of extrahepatic depositions described by Prince et al. (1.1-41.3 mL) [145]. The same holds for the activity, where our extrahepatic depositions contained $0.35 \%, 0.76 \%$ and $1.52 \%$ of the total activity, while Prince et al. found extrahepatic deposition activity ranging from $0.1 \%$ to $19.5 \%$. Although based on this phantom experiment we cannot state that all extrahepatic depositions found by Prince et al. (on ${ }^{99 m} \mathrm{mc}-\mathrm{MAA}$ SPECT) would be visible on a $100 \mathrm{MBq}{ }^{90} \mathrm{Y}$ SPECT, the majority of these extrahepatic depositions would likely be visible, because our phantom was on the lower end of both volume and activity, and we can assume that larger and/or more active extrahepatic depositions will be easier to visualise.

Due to the single phantom configuration, this study only investigated image quality of a phantom representing a radioembolization patient with a $\mathrm{T} / \mathrm{N}$ of 7.9. A wide range of T/N has been reported in patients $[159,160]$. The results from this study cannot be directly extrapolated to distributions with other $\mathrm{T} / \mathrm{N}$, although the CNR of the tumours would most likely go down for tumours with a lower T/N.

Another limitation of the phantom is that the activity distribution within the phantom compartments was uniform, and did not represent a clinical setting. Therefore, we included a patient scan to evaluate the image quality for a more inhomogeneous activity distribution. There is no ground truth distribution for the patient scan, however, compared to the long acquisition time scan, results were comparable.

The SPECT data were reconstructed with an MC-based reconstruction algorithm, which is currently not available for clinical practice. However, the image quality of the ${ }^{90} \mathrm{Y}$ SPECT scan relies heavily on the scatter correction offered by the MC-based reconstruction model, which has also been implemented by other groups $[18,161]$. For availability of the MC-based reconstruction algorithm, please contact the authors. 
Chapter 6. Feasibility of imaging ${ }^{90}$ y microspheres at diagnostic activity levels

A next step toward implementation of a ${ }^{90}$-based pre-treatment procedure would be a patient study with several patients who receive both a pre-treatment ${ }^{99 m}$ TC-MAA SPECT and a pre-treatment ${ }^{90} \mathrm{Y}$ SPECT for comparison. Another option is to simulate the pretreatment ${ }^{90}$ Y SPECT using a short acquisition time as was done in this study.

\section{Conclusion}

In this phantom study, a ${ }^{90} \mathrm{Y}$ pre-treatment SPECT/CT scan (100 MBq), reconstructed with

a Monte Carlo-based reconstruction model, was found to have a similar quantitative accuracy as ${ }^{99 \mathrm{~m} T c-M A A}$ SPECT/CT in estimating LSF, T/N, parenchymal, and tumour dose, while it can also visualise extrahepatic depositions. The image quality of a simulated ${ }^{90}$ Y pre-treatment scan of a patient was visually and quantitatively similar to the posttreatment scan. 


\section{Chapter 7}

Comparison of the Biograph Vision and Biograph $\mathrm{mCT}$ for quantitative ${ }^{90} \mathrm{Y}$ PET/CT imaging for radioembolization

Published as:

B. Kunnen, C. Beijst, M.G.E.H. Lam, M.A. Viergever, H.W.A.M. de Jong.

Comparison of the Biograph Vision and Biograph mCT

for quantitative Physics, 7 (1): 14; (2020) 


\section{Abstract}

Background: New digital PET scanners with improved time of flight timing and extended axial field of view such as the Siemens Biograph Vision have come on the market and are expected to replace current generation photomultiplier tube (PMT)-based systems such as the Siemens Biograph $\mathrm{mCT}$. These replacements warrant a direct comparison between the systems, so that a smooth transition in clinical practice and research is guaranteed, especially when quantitative values are used for dosimetry-based treatment guidance. The new generation digital PET scanners offer increased sensitivity. This could particularly benefit ${ }^{90} \mathrm{Y}$ imaging, which tends to be very noisy owing to the small positron branching ratio and high random fraction of ${ }^{\circ} \mathrm{Y}$. This study aims to determine the ideal reconstruction settings for the digital Vision for quantitative ${ }^{90} Y$ imaging and to evaluate the image quality and quantification of the digital Vision in comparison with its predecessor, the PMT-based $\mathrm{mCT}$, for ${ }^{90} \mathrm{Y}$ imaging in radioembolization procedures.

Methods: The NEMA image quality phantom was scanned to determine the ideal reconstruction settings for the Vision. In addition, an anthropomorphic phantom was scanned with both the Vision and the $\mathrm{mCT}$, mimicking a radioembolization patient with lung, liver, tumour, and extrahepatic deposition inserts. Image quantification of the anthropomorphic phantom was assessed by the lung shunt fraction, the tumour to non-tumour ratio, the parenchymal dose, and the contrast to noise ratio of extrahepatic depositions.

Results: For the Vision, a reconstruction with 3 iterations, 5 subsets and no postreconstruction filter is recommended for quantitative ${ }^{90} Y$ imaging, based on the convergence of the recovery coefficient. Comparing both systems showed that the noise level of the Vision is significantly lower than that of the $\mathrm{mCT}$ (background variability of $14 \%$ for the Vision and $25 \%$ for the $\mathrm{mCT}$ at $2.5 \cdot 10^{3} \mathrm{MBq}$ for the $37 \mathrm{~mm}$ sphere size). For quantitative ${ }^{90} \mathrm{Y}$ measures, such as needed in radioembolization, both systems perform similarly.

Conclusions: We recommend to reconstruct ${ }^{90} \mathrm{Y}$ images acquired on the Vision with 3 iterations, 5 subsets and no post-reconstruction filter for quantitative imaging. The Vision provides a reduced noise level, but similar quantitative accuracy as compared with its predecessor the $\mathrm{mCT}$.

\section{Introduction}

New digital positron emission tomography/computed tomography (PET/CT) scanners, such as the GE Discovery MI and the Siemens Biograph Vision, offer better time of flight (TOF) performance and a larger axial field of view (FOV) for higher effective sensitivity than their photomultiplier tube (PMT)-based counterparts. This increased sensitivity can be used to shorten acquisition time, reduce radionuclide activity and/or improve image quality. It is likely that hospitals will gradually switch to these next generation digital PET/ CT scanners.

The higher effective sensitivity of new digital PET/CT scanners could particularly benefit yttrium-90 (90Y) imaging, which tends to be very noisy owing to the small positron branching ratio. PET is often regarded as the preferred imaging modality for ${ }^{90} \mathrm{Y}$ imaging since it offers higher resolution than Bremsstrahlung single photon emission computed tomography (SPECT) $[100,152]$. It is used to image the distribution of ${ }^{90} Y$ microspheres after radioembolization treatment. Clinical relevant features of a post-treatment ${ }^{90} \mathrm{Y}$ PET scan include the lung shunt fraction (LSF), presence of extrahepatic depositions, and intrahepatic dose distribution [152,162]. At the time of imaging, ${ }^{90} \mathrm{Y}$ activities in the liver typically range from 500-5000 MBq [34,163-165]. Since radioembolization and the dosimetry involved constitute a relatively new clinical area, ${ }^{90} \mathrm{Y}$ imaging is often performed in the setting of research studies, where ${ }^{90} \mathrm{Y} \mathrm{PET} \mathrm{is} \mathrm{used} \mathrm{in} \mathrm{dose-response}$ studies $[45,124,163,166]$. Consistency of quantitative measurements is key throughout an imaging study, and a change of scanner during the study may affect this consistency. To warrant study consistency, a good understanding of the ${ }^{90} Y$ imaging properties of both systems is required.

The higher sensitivity of new digital PET/CT scanners may open doors to new applications. Recently, we proposed the use of a low dosage of ${ }^{90} \mathrm{Y}$ microspheres ( 100 MBq) for a pre-treatment radioembolization procedure for therapy planning purposes $[140,141]$. Currently, pre-treatment radioembolization procedures are performed with technetium$99 \mathrm{~m}\left({ }^{99 \mathrm{~m} T C)}\right.$ labelled macroaggregated albumin (MAA). Because of the differences in shape and size between the MAA particles and the microspheres, differences between estimated dose distribution by MAA and the true dose distribution have been reported [34,42,44,167]. These differences could be minimised by using the same particle for treatment and pre-treatment procedure, as has been shown for holmium-166 $\left({ }^{166} \mathrm{Ho}\right)$ microsphere radioembolization [47]. A safe dosage for the pre-treatment ${ }^{166} \mathrm{Ho}$ microsphere procedure is $250 \mathrm{MBq}[144,145]$. Inasmuch as the total energy absorbed per $\mathrm{Bq}$ is higher for ${ }^{90} \mathrm{Y}$ than for ${ }^{166} \mathrm{Ho}$, this dosage would translate to $100 \mathrm{MBq}$ of ${ }^{90} \mathrm{Y}$ to avoid unintended radiation damage $[29,140,141]$ 
The low activity of ${ }^{90} Y$ for the pre-treatment procedure makes imaging challenging. Using $100 \mathrm{MBq}$, Bremsstrahlung SPECT can produce quantitative images to accurately estimate the LSF, but images are of low resolution [140]. PET overestimates the LSF at $100 \mathrm{MBq}$ [140]. This is caused by the low count statistics and the high random fraction, which result in a positive bias and high noise levels in the PET reconstruction. This makes PET unsuitable as an imaging modality for imaging the dose distribution of a pre-treatment ${ }^{90} \mathrm{Y}$ procedure.

The previous study that showed the infeasibility of PET as an imaging modality for a ${ }^{90} Y$ pre-treatment procedure, was performed with the PMT-based Biograph mCT PET system (Siemens) [140]. It's successor, the digital Biograph Vision, has improved spatial resolution, improved timing resolution, an extended axial FOV, and increased sensitivity by $70 \%$ measured at the centre of the transaxial FOV (Table 7.1) [168]. When comparing fluorine-18 ( $\left.{ }^{(18} \mathrm{F}\right)$ fluorodeoxyglucose images of oncological patients from both systems, the Vision scored higher in terms of overall image quality and image noise, than the $\mathrm{mCT}$ [169].

Table 7.1: Technical specifications of Biograph Vision and Biograph $\mathrm{mCT}$. LSO = lutetium oxyorthosilicate, FOV = field of view, FWHM = full width at half maximum.

\begin{tabular}{lcc}
\hline & Vision[168] & mCT[170] \\
\hline Crystals & LSO, 3.2 $3.2 \times 20 \mathrm{~mm}$ & LSO, $4.0 \times 4.0 \times 20 \mathrm{~mm}$ \\
Detector elements & Silicon photomultipliers & Photomultiplier tubes \\
Axial FOV & $26.1 \mathrm{~cm}$ & $22.1 \mathrm{~cm}$ \\
TOF timing resolution & $210-215 \mathrm{ps}$ & $540 \mathrm{ps}$ \\
Transverse spatial resolution (measured at $1 \mathrm{~cm}$ & $3.7 \mathrm{~mm}$ (FWHM) & $4.4 \mathrm{~mm}$ (FWHM) \\
vertically from the centre of the FOV with ${ }^{8 \mathrm{~F}}$ ) & $16.4 \mathrm{kcps} / \mathrm{MBq}$ & $10.0 \mathrm{kcps} / \mathrm{MBq}$ \\
Sensitivity (according to NEMA NU-2 2012) & $4.7 \mathrm{~ns}$ & $4.1 \mathrm{~ns}$ \\
Time coincidence window & $435-585 \mathrm{keV}$ & $435-650 \mathrm{keV}$ \\
Energy window & $49 \%$ & $43 \%$ \\
Bed overlap & &
\end{tabular}

The improvements of the Vision with regard to the $\mathrm{mCT}$ are expected to lead to improved ${ }^{90} Y$ imaging. The QUEST phantom study [157] has extensively studied the performance of multiple PET systems for ${ }^{90} \mathrm{Y}$ imaging and recommends reconstruction settings for quantitative purposes for the systems involved. However, the Vision was not part of the QUEST study and to our knowledge there are no recommended reconstruction settings for this system regarding quantitative ${ }^{90} Y$ imaging.
The purpose of the present study is to evaluate the performance of the Siemens Biograph Vision in comparison with its PMT-based counterpart, the $\mathrm{mCT}$, for ${ }^{90} \mathrm{Y}$ imaging. We used the NEMA phantom to determine the ideal reconstruction settings for the Vision in analogy to the QUEST study [157]. These reconstruction settings for the Vision were compared with the recommended reconstruction setting of the MCT [157] in terms of standardized image quality metrics. In addition, clinical relevant features for radioembolization were compared by using an anthropomorphic phantom.

\section{Methods}

\section{Phantoms}

Two phantoms were used for this study: the NEMA image quality phantom (PTW, Freiburg, Germany) and a modified anthropomorphic thorax phantom (model ECT/TOR/P, IEL, Chilcompton, UK). The NEMA phantom is used to compare standardised image quality metrics in analogy with the QUEST study [157]. The thorax phantom is used to simulate a radioembolization patient and to evaluate the accuracy of lung shunt estimation, extrahepatic deposition visibility, and intrahepatic activity distribution.

The NEMA phantom consists of a $9.7 \mathrm{~L}$ torso-shaped compartment containing six fillable spheres (inner diameter of 10,13, 17, 22, 28 and $37 \mathrm{~mm}$ ) and a cold, cylindrical lung insert. It was prepared in a similar way as in the QUEST study protocol [157], where the phantom was filled with ${ }^{90} \mathrm{Y}$ chloride, in $0.5 \mathrm{M} \mathrm{HCl}$ to prevent adhesion to the plastic phantom walls [153], to acquire a sphere-to-background concentration ratio of 8 and a total initial activity of $2.5 \cdot 10^{3} \mathrm{MBq}$.

The modified thorax phantom consists of a torso-shaped compartment containing a liver compartment with a solid tumour (sphere of $15.9 \mathrm{~mL}$ ) and a necrotic tumour (outer rim $18.9 \mathrm{~mL}$ and inner sphere $5.6 \mathrm{~mL}$ ), two lung compartments, a cylindrical spine insert, and three extrahepatic compartments (spheres of 2.0, 4.2 and $8.2 \mathrm{~mL}$ ). The phantom was filled with ${ }^{90} \mathrm{Y}$ chloride in $0.5 \mathrm{M} \mathrm{HCl}$, to acquire a lung shunt fraction (LSF) of $5.0 \%$, a tumour to non-tumour ratio (T/N) of 8.0 and a total initial activity of $1.0 \cdot 10^{3} \mathrm{MBq}$. The activity concentration of the extrahepatic depositions in the phantom was based on the median size ( $6.8 \mathrm{~mL}$, range $1.1-42 \mathrm{~mL}$ ) and median activity (1.3\% of the infused activity) of 34 extrahepatic depositions found by Prince et al. [145], and was therefore chosen to be: 1.3\% of the total activity in the phantom / $6.8 \mathrm{~mL}$ 


\section{Image acquisition}

Both phantoms were scanned consecutively on a Siemens Biograph $\mathrm{mCT}$ and a Siemens Biograph Vision. Table 7.1 lists technical specifications of both systems. Scans were acquired during decay and all activities at the time of imaging are listed in Table 7.2.

Table 7.2: Total activity (MBq) in the NEMA and thorax phantoms at time of imaging.

\begin{tabular}{|c|c|c|c|c|c|c|c|c|}
\hline & & Day 0 & Day 2 & Day 4 & Day 6 & Day 8 & Day 9 & Day 10 \\
\hline \multirow{2}{*}{ NEMA } & $\mathrm{mCT}$ & 2511 & 1560 & 901 & 537 & 328 & 259 & 194 \\
\hline & Vision & 2563 & 1526 & 930 & 553 & 322 & 264 & 205 \\
\hline \multirow{2}{*}{ thorax } & $\mathrm{mCT}$ & 961 & 597 & 345 & 206 & 126 & 99 & 74 \\
\hline & Vision & 982 & 585 & 356 & 212 & 123 & 101 & 78 \\
\hline
\end{tabular}

Both phantoms were scanned in two bed positions of 15 minutes per bed position on both systems. A CT scan was made for attenuation correction and to support delineation.

\section{Image reconstruction}

Images acquired by the $\mathrm{mCT}$ were reconstructed with the reconstruction settings recommended by the QUEST study [157]. This is an ordered subset expectation maximization (OSEM) reconstruction algorithm, including time of flight (TOF) information, resolution recovery (TrueX), attenuation, scatter, random, dead time, and decay correction. The reconstruction used 2 iterations with 21 subsets, resulting in a voxel size of $4.1 \times 4.1 \times$ $3.0 \mathrm{~mm}^{3}$ and no post-reconstruction filter was applied.

Since, to our knowledge, no prior study has been published on ${ }^{90}$ Y PET imaging using the Vision, images acquired at the Vision were reconstructed with a variety of reconstruction settings, so as to determine the optimal setting. All reconstruction methods used an OSEM reconstruction algorithm, including TOF information, resolution recovery (TrueX), attenuation, scatter, random, dead time, and decay correction. The number of subsets is fixed at 5 by the vendor and the number of iterations was varied between 1 and 17 . Images were reconstructed on a 220x220 matrix resulting in a voxel size of $3.3 \times 3.3 \times 3.0 \mathrm{~mm}^{3}$. No post-reconstruction filter was applied. The optimal reconstruction was determined based on convergence of the recovery coefficient with iteration number.

\section{Analysis}

For the NEMA image quality phantom we analysed the same metrics as the QUEST phantom study. These are:

Percent background variability (BV), following the NEMA NU 2-2007 guidelines, defined for each sphere size as:

$$
B V=\frac{S D_{B, S}}{C_{B, S}} * 100 \%
$$

where $\mathrm{SD}_{\mathrm{BS}}$ is the standard deviation of the average of the 60 background regions of interest (ROIs) for sphere size $s$ and $C_{B, s}$ is the average count of the 60 background ROls for sphere size $\mathrm{s}$.

Percentage misplaced counts in the lung insert $\left(\Delta C_{\text {lung }}\right.$ ), following the NEMA NU 2-2007 guidelines, defined as:

$\Delta C_{\text {lung }}=\frac{C_{\text {lung }}}{C_{B}} * 100 \%$

where $C_{\text {lung }}$ is the average count in the lung insert $\mathrm{ROI}$ and $\mathrm{C}_{B}$ is the average count of the background ROIs.

Background concentration accuracy (BCA), defined for each sphere size as:

$B C A=\frac{a_{B, \text { measured }}-a_{B, \text { true }}}{a_{B, \text { true }}} * 100 \%$

where $\mathrm{a}_{\mathrm{B} \text {,measured }}$ is the measured activity concentration in the background and $\mathrm{a}_{\mathrm{B}, \mathrm{true}}$ is the true activity concentration in the background.

Total activity accuracy (TAA), defined as:

$T A A=\frac{A_{\text {TOT,measured }}-A_{\text {TOT }, \text { true }}}{A_{\text {TOT }, \text { true }}} * 100 \%$

where $A_{\text {roT,measured }}$ is the measured total activity in the entire FOV and $A_{\text {roTt,true }}$ is the known true total activity in the phantom

Recovery coefficient (RC), following the NEMA NU 2-2007 guidelines for delineation of the spheres, defined for each sphere size as:

$R C=\frac{a_{S, \text { measured }}}{a_{S, \text { tue }}} * 100 \%$ 
where $a_{5, \text { measured }}$ is the measured activity concentration in the sphere and $a_{S, t r u e}$ is the true activity concentration in the sphere.

To evaluate the thorax phantom we assessed the following metrics, which are commonly assessed for radioembolization treatment planning:

The lung shunt fraction (LSF), defined as:

$L S F=\frac{C_{\text {lung }}}{C_{\text {lung }}+C_{\text {liver }}} * 100 \%$

where $C_{\text {lung }}$ is the total count in the lungs dilated by $6 \mathrm{~mm}$ and $C_{\text {liver }}$ is the total count in the liver dilated by $6 \mathrm{~mm}$. The lung and liver contours were dilated by the spatial resolution of the PET systems to partially compensate for the partial volume effect.

Tumour to non-tumour ratio (T/N) of the solid and the necrotic tumour, defined as:

$$
T / N=\frac{C_{\text {tumour }}}{C_{\text {parenchyma }}}
$$

where $C_{\text {tumour }}$ is the average count in the tumour volume of interest $(\mathrm{VOI})$ and $\mathrm{C}_{\text {parenchyma }}$ is the average count in the parenchymal VOI

Parenchymal dose per $\mathrm{GBq}$ of ${ }^{90} \mathrm{Y}$ administered, defined as:

$D_{\text {parenchyma }}=\frac{\frac{A_{\text {parenchyma }}}{A_{\text {total }}} * 50}{m_{\text {parenchyma }}}$

where $A_{\text {parenchyma }}$ is the activity in the parenchymal $\mathrm{VOI}, \mathrm{A}_{\text {total }}$ is the known total activity in the phantom, $m_{\text {parenchyma }}$ is the mass of the parenchymal VOI (determined using the liver VOI volume and a conversion factor of $1.03 \mathrm{~g} / \mathrm{mL}$ ), and fifty is the absorbed energy in joule from the decay of $1 \mathrm{GBq}$ of ${ }^{90} \mathrm{Y}$.

Contrast-to-noise ratio (CNR) of the extrahepatic depositions as a measure of visibility, defined as:

$C N R=\frac{C_{\text {deposition }}-C_{B}}{S D_{B}}$

where $C_{\text {deposition }}$ is the average count in the extrahepatic deposition VOI, $C_{B}$ is the average count in a background $\mathrm{VOI}$ and $\mathrm{SD}_{B}$ is the standard deviation of a background VOI.
Differences between the Vision and the $\mathrm{mCT}$ are tested for significance with a paired t-test assuming a $5 \%$ significance level.

\section{Results}

Figure 7.1 shows the total prompts, randoms, and net trues for the Vision and $\mathrm{mCT}$ acquisitions of the NEMA and thorax phantoms. The net trues show a strong linear trend with activity, of which the slope, intercept, and coefficient of determination are listed in Table 7.3. The Vision has an increased trues rate compared with the mCT by 68\% (NEMA phantom) and 65\% (thorax phantom).

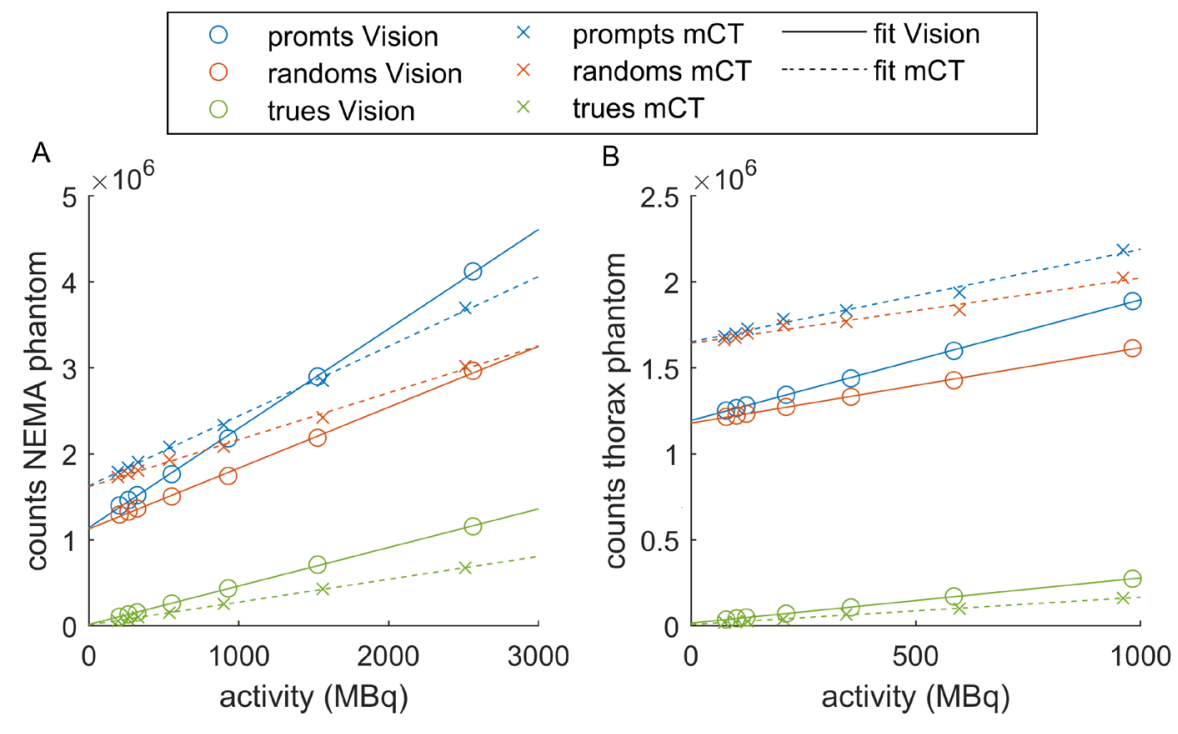

Figure 7.1: Total prompts, randoms, and net trues for a range of ${ }^{90} \mathrm{Y}$ activities for the Vision and $\mathrm{mCT}$ acquisitions of the NEMA phantom (a) and the thorax phantom (b). The solid and dashed lines are linear fits of the data for the Vision and the $\mathrm{mCT}$ respectively.

Table 7.3: Slope, intercept, and coefficient of determination $\left(R^{2}\right)$ of the linear fit of the net true counts in the sinograms. Numbers between brackets are the 95\% confidence intervals.

\begin{tabular}{lccc}
\hline Measurement & Slope (counts/MBq) & Intercept (counts) & $\mathbf{R}^{\mathbf{2}}$ \\
\hline NEMA Vision & $448[439-457]$ & $1.57 \cdot 10^{4}\left[5.16 \cdot 10^{3}-2.623 \cdot 10^{4}\right]$ & 0.9997 \\
NEMA mCT & $266[261-271]$ & $1.04 \cdot 10^{4}\left[4.53 \cdot 10^{3}-1.62 \cdot 10^{4}\right]$ & 0.9997 \\
Thorax Vision & $263[259-267]$ & $1.58 \cdot 10^{4}\left[1.37 \cdot 10^{4}-1.78 \cdot 10^{4}\right]$ & 0.9998 \\
Thorax mCT & $159[152-167]$ & $0.762 \cdot 10^{4}\left[4.15 \cdot 10^{3}-1.11 \cdot 10^{4}\right]$ & 0.9983 \\
\hline
\end{tabular}




\section{Ideal reconstruction settings Vision}

Figure 7.2 shows the transverse slice of the NEMA phantom at day $0\left(2.5 \cdot 10^{3} \mathrm{MBq}\right)$ for three reconstruction settings for the Vision acquisition and for the 2-iterations setting for the $\mathrm{mCT}$ acquisition. Visually the reconstructions of the Vision outperform the reconstruction of the $\mathrm{mCT}$ because of the lower noise level and the better visibility of the (smaller) spheres. Increasing the number of iterations increases noise.

Figure 7.3 shows the recovery coefficient and the percentage background variability plotted against iteration number of the different sphere diameters at day 0 . Depending on the study purpose, one can either choose a number of iterations that favours high recovery (higher number of iterations) or one that favours low noise (low number of iterations). Since the purpose of this study is quantitative imaging, we like to achieve a high RC. The RC curves of the three largest spheres shown in Figure 7.3 converge at 3 iterations. The RC curves of the three smallest spheres show more varying trends. In order to not increase the background variability while the recovery coefficient barely improves, we choose 3 iterations with 5 subsets as the ideal reconstruction setting for the Vision. For subsequent comparisons between the Vision and the $m C T$, the images acquired by the Vision will therefore be reconstructed using 3 iterations with 5 subsets without a post-reconstruction filter.

\section{Comparison Vision and $\mathbf{m C T}$}

Figure 7.4 compares the NEMA phantom scanned at the Vision (reconstructed with 3 iterations, 5 subsets) with the $\mathrm{mCT}$ scans (reconstructed with 2 iterations, 21 subsets). Paired t-tests showed a significant difference between the Vision and the $\mathrm{mCT}$ for background variability (BV) ( $p$-value $<0.001$ ) and background concentration accuracy $(p$-value $<0.05)$. The BV was about twice as high for the $\mathrm{mCT}$. This affects the visibility of hot and cold spots. For other quantitative measures there was no significant difference between the Vision and the $\mathrm{mCT}$.

The thorax phantom mimics a radioembolization patient and has an activity distribution that is clinically more relevant than that of the NEMA phantom. Figure 7.5 shows metrics that are commonly assessed for radioembolization treatment planning. Paired t-tests showed a significant difference between both systems for the LSF ( $p$-value $<0.05)$, the parenchymal dose ( $p$-value $<0.05$ ), and the CNR ( $p$-value $<0.001$ ). Although significant differences were observed, both systems showed similar trends for these metrics. Both overestimated the LSF, and as expected, the overestimation got worse when the total activity decreased. This can be attributed to the earlier described positive bias in maximum likelihood reconstructions at low activities $[140,156]$. However, unexpectedly, the Vision showed a slightly larger overestimation of the LSF than the $\mathrm{mCT}$. Metrics regarding intrahepatic dose distribution (T/N and parenchymal dose) were underestimated by both systems and showed large variations. The CNR, a measure for the visibility of the extrahepatic depositions, showed the most stable trend and decreased with decreasing phantom activity. The CNR was considerably higher for the Vision than for the $\mathrm{mCT}$.
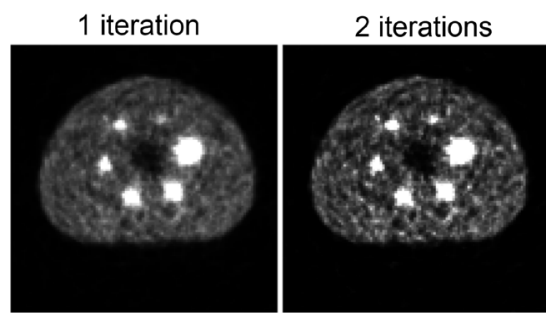

3 iterations
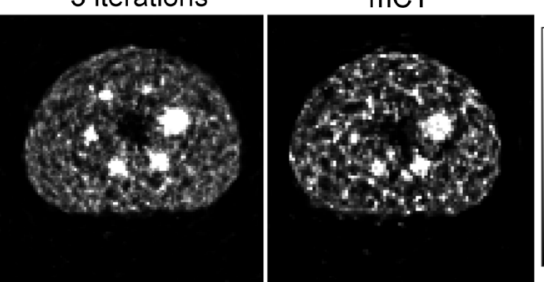

Figure 7.2: Transverse slice of three Vision reconstructions (1, 2, 3 iterations with 5 subsets) and $\mathrm{mCT}$ reconstruction (2 iterations with 21 subsets) of the NEMA phantom at day $0\left(2.5 \cdot 10^{3} \mathrm{MBq}\right)$, centred on the spheres. Images are scaled between 0 and $1 \mathrm{MBq} / \mathrm{mL}$.
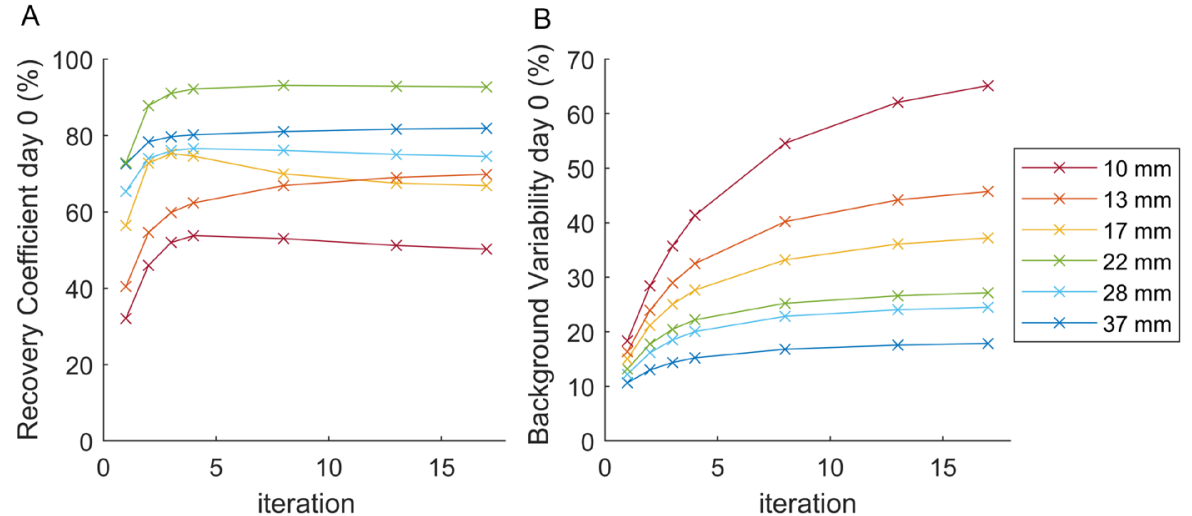

Figure 7.3: Recovery coefficient (a) and percentage background variability (b) of the NEMA phantom spheres with varying diameter at day $0\left(25.10^{3} \mathrm{MBq}\right.$ ) as a function of iteration number. 

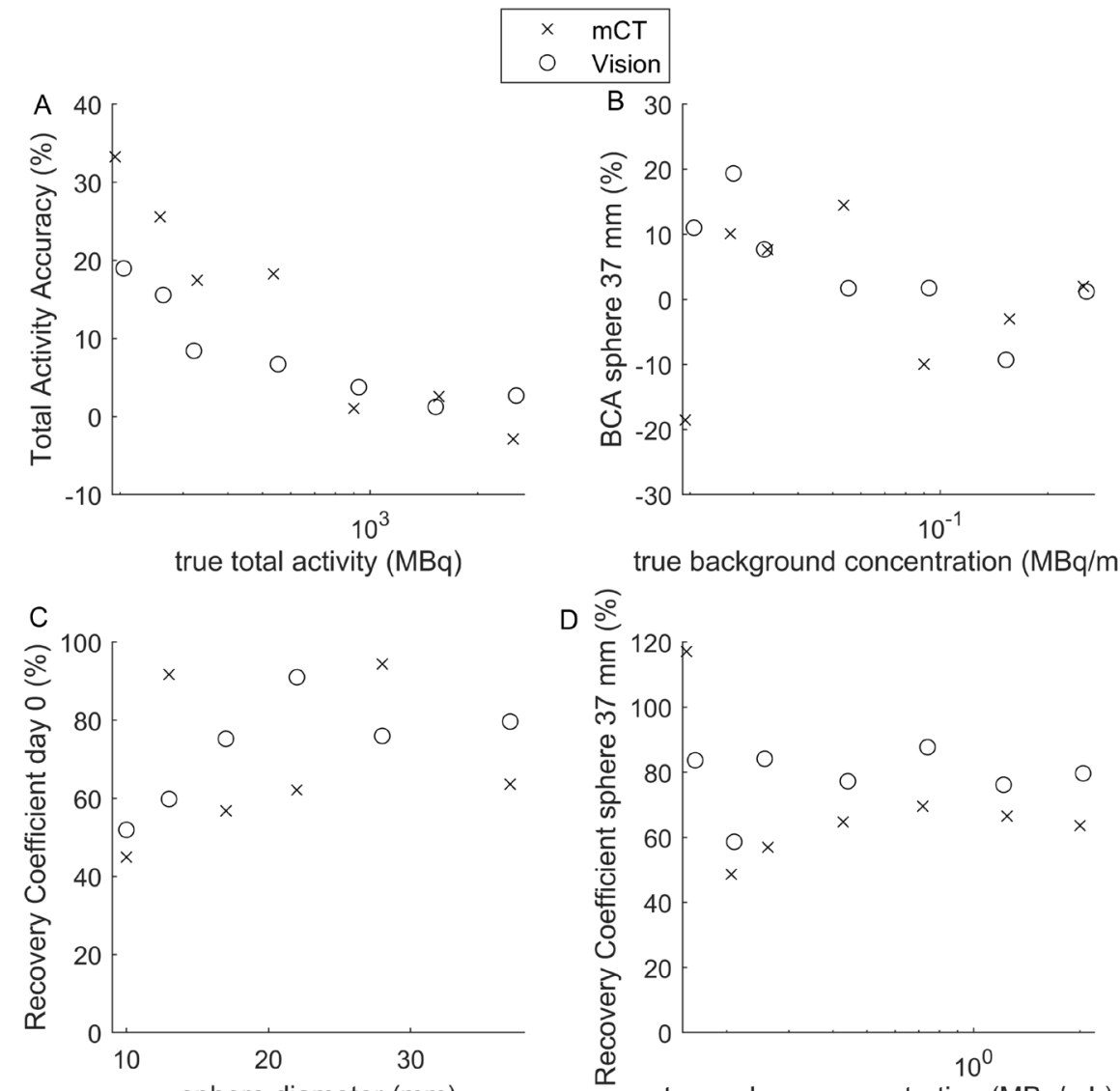

$\mathrm{D} \stackrel{\mathrm{o}}{\mathrm{a}}$

true background concentration $(\mathrm{MBq} / \mathrm{mL})$
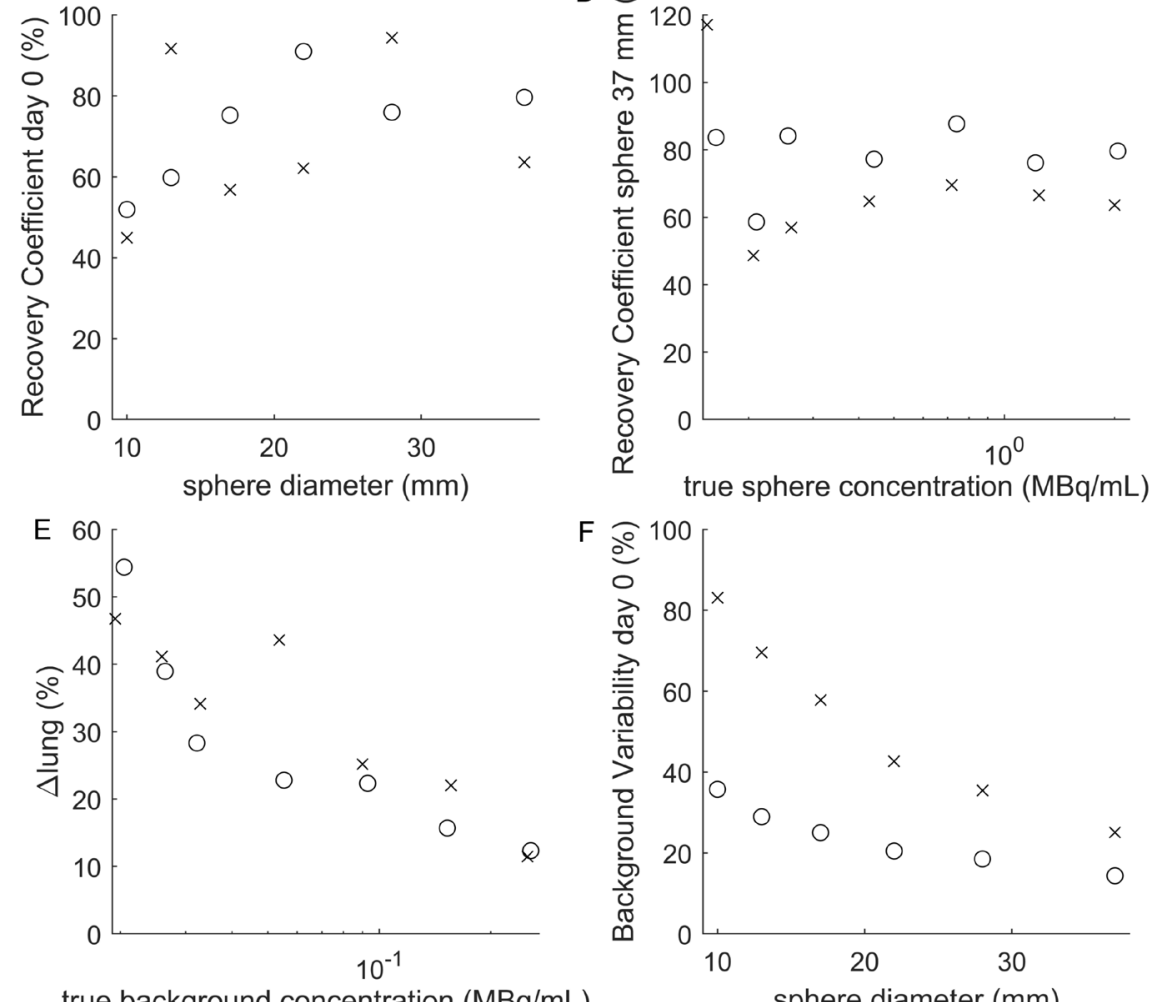

true background concentration $(\mathrm{MBq} / \mathrm{mL})$

sphere diameter $(\mathrm{mm})$

Figure 7.4: Results of the NEMA phantom comparing the $\mathrm{mCT}$ ( 2 iterations, 21 subsets) with the Vision (3 iterations, 5 subsets). BCA = background concentration accuracy.
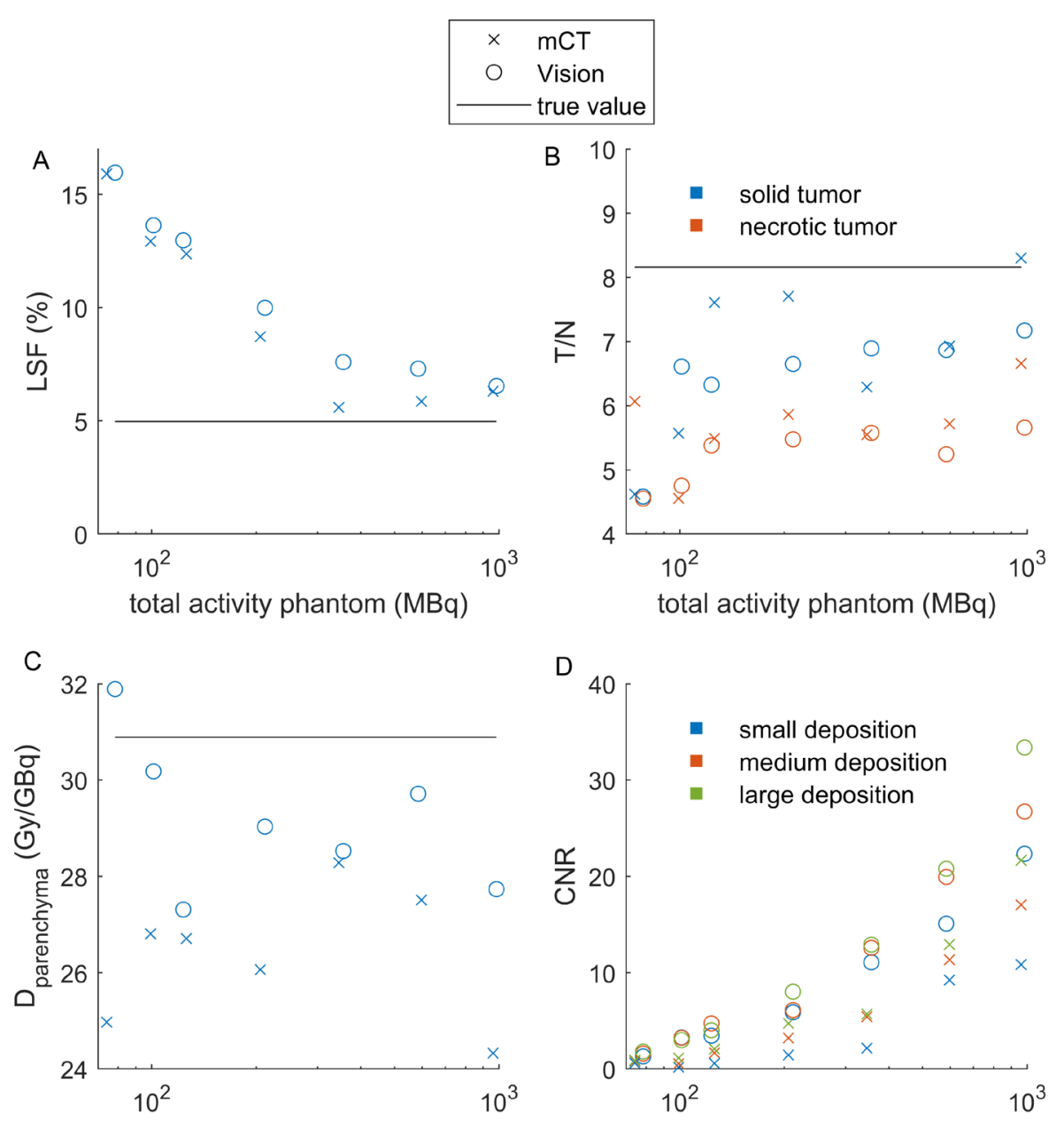

total activity phantom $(\mathrm{MBq})$

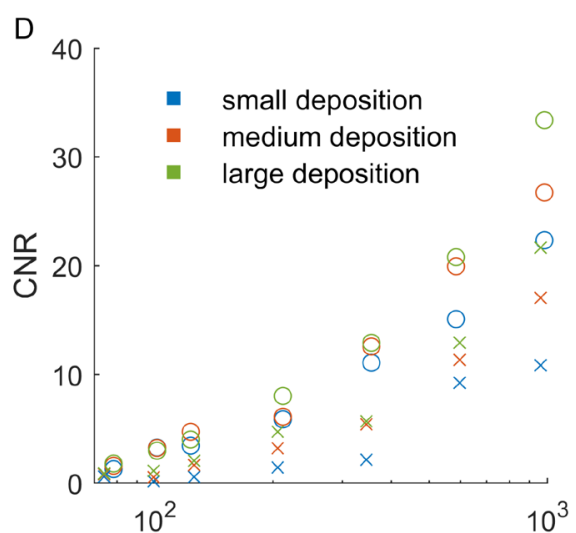

total activity phantom $(\mathrm{MBq})$

Figure 7.5: Performance of the Vision and the $\mathrm{mCT}$ for measures concerning radioembolization treatment planning: (a) lung shunt fraction (LSF), (b) tumour to non-tumour ration (T/N), (c) parenchymal dose (Dparenchymal), and (d) contrast-to-noise ratio (CNR) of the extrahepatic depositions.

\section{Discussion}

In this study we evaluated the performance of the Vision for ${ }^{90} Y$ imaging and compared it with the performance of its predecessor, the $\mathrm{mCT}$. We determined the optimal reconstruction settings for the Vision to be 3 iterations with 5 subsets. We found that the Vision outperformed the $\mathrm{mCT}$ in terms of noise level, but performed similarly in terms of quantitative accuracy. 
The prompts, randoms, and trues of both the Vision and the $\mathrm{mCT}$ show good linearity, which implies that no saturation of the detectors occurs. The Vision had 65\% (thorax phantom) or $68 \%$ (NEMA phantom) more net trues than the $\mathrm{MCT}$. This is in agreement with the $70 \%$ increase in sensitivity found by van Sluis et al. for ${ }^{18} \mathrm{~F}[168]$. The slope of the true count with activity is dependent on the geometry and activity distribution of the scanned object, since the slopes of the true count with activity on the same scanner with the same acquisition parameters differ between NEMA and thorax phantoms. The total prompts and randoms have an offset due to the presence of lutetium-176 $\left({ }^{176} \mathrm{Lu}\right)$ in the crystals. The magnitude of this offset is dependent on e.g., the amount of crystals (and therefore the amount of ${ }^{176} \mathrm{Lu}$ ), the number of possible coincidence line-of-response, the time coincidence window and the energy window [171]. All these specifications differ between the $\mathrm{mCT}$ and the Vision and result in a larger offset for the $\mathrm{MCT}$ than for the vision.

Scans at both systems were acquired in step-and-shoot mode, instead of continuoustable-motion mode. For scans with a short scan length and long acquisition time, like ${ }^{90}$ Y PET liver scans, it has been shown that step-and-shoot mode has a higher counting efficiency compared to continuous-table-motion mode [172]. Therefore, we chose to perform all scans in step-and-shoot mode to obtain the best image quality and quantification precision.

The ideal reconstruction setting is dependent on the study purpose. Just like the QUEST study, we focused on quantitative accuracy. We recommend to reconstruct ${ }^{90} Y$ data acquired on the Vision with 3 iterations, 5 subsets and no post-reconstruction filter. This is based on the fact that after three iterations quantitative metrics will only marginally improve while noise will still substantially increase. When the objective of a scan is focused on visual image quality, one could decide to add a post-reconstruction filter. In this study, we did not study the influence of different filters in detail, since Gaussian filters will reduce quantitative accuracy.

The results from our NEMA measurements on the $\mathrm{MCT}$ were comparable with the results from the QUEST study. There were some small deviations (like the steep trend seen for our background variability versus sphere diameter, which is less pronounced in the QUEST study) but these can be explained by the fact that the data represented by QUEST consisted of multiple scanners. The measurements on our single scanner still fall within the error margins of the QUEST study.
Comparing quantitative results of the Vision with the $\mathrm{mCT}$ does not show clinically relevant differences between the systems. This suggests that a smooth upgrade during quantitative studies is possible, if the proper reconstruction protocol is chosen. However, the noise level of the Vision is substantially lower than the noise lever of the mCT. This could make visualization of hot and/or cold regions, such as extrahepatic depositions, easier on the Vision. Van Sluis et al. shows similar results comparing the Vision and the $\mathrm{mCT}$ for ${ }^{18} \mathrm{~F}$, where they show that the Vision outperforms the $\mathrm{mCT}$ visually, but based on quantitative measures both systems are comparable [169].

For the specific clinical case of ${ }^{90} Y$ imaging for radioembolization, quantitative measures are important. Although the LSF and parenchymal dose show significant differences between the Vision and the $\mathrm{mCT}$, these differences are small (mean LSF difference is 0.9 percent point (pp), mean parenchymal dose difference is $2.8 \mathrm{~Gy} / \mathrm{GBq}$ ). Furthermore, the uncertainty in volume measurement and uncertainty in the activity calibration, cause an uncertainty of $\pm 10 \%$ in the true parenchymal dose of the thorax phantom. This makes it difficult to conclude with certainty whether the Vision or the $\mathrm{mCT}$ results in a more accurate estimated parenchymal dose. With a ${ }^{90} \mathrm{Y}$ pre-treatment procedure in mind, errors in estimated parenchymal dose could cause under- or overdosage. In combination with the overestimation of the LSF by $9 \mathrm{pp}$ at $100 \mathrm{MBq}$, we have to conclude that the Vision is, just like its predecessor the $\mathrm{mCT}$, unsuitable as an imaging system for imaging of a theoretically safe pre-treatment dosage of $100 \mathrm{MBq}{ }^{90} \mathrm{Y}$.

The Vision does have a lower noise level compared with the $\mathrm{mCT}$. This means that the Vision would be the more appropriate system when addressing the dose distribution visually, for example after radioembolization treatment to identify whether the dose distribution in a tumour is homogenous or not.

\section{Conclusion}

In this study we evaluated the performance of the Vision for ${ }^{90} Y$ imaging and compared it with the performance of its predecessor, the $\mathrm{mCT}$. We recommend to reconstruct ${ }^{90} \mathrm{Y}$ images acquired on the Vision with 3 iterations, 5 subsets and no post-reconstruction filter, for quantitative purposes. Visually, the Vision outperforms the $\mathrm{mCT}$ because of its lower noise level, but based on quantitative measurements both scanners perform similarly. 


\section{Chapter 8}

\section{General discussion}

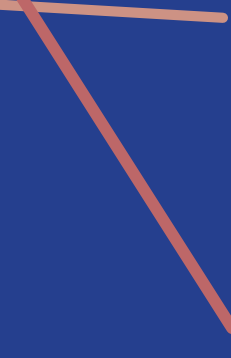


Radioembolization may profit from accurate dosimetry, similarly to radiotherapy. However, accurate dosimetry in radioembolization is hampered by a number of factors, including (i) the separate pre-treatment and treatment procedures which are days apart, thereby introducing potential poor matching of the catheter position; and (ii) the use of ${ }^{99 \mathrm{mT} \mathrm{T}-}$ MAA for the pre-treatment procedure, which inaccurately mimics the ${ }^{90} \mathrm{Y}$ microsphere distribution. A solution to this second problem is replacing the ${ }^{90} \mathrm{Y}$ microsphere/99mTCMAA tandem with the ${ }^{166} \mathrm{Ho}$ microsphere, which can be used for both the low-dose pre-treatment and the high-dose treatment procedures. In this thesis, two additional solutions were suggested and investigated. The first is the introduction of a dual layer scanner for simultaneous hybrid nuclear and fluoroscopic imaging in the intervention room. This scanner could guide the physician during the pre-treatment procedure and perhaps combine the pre-treatment and treatment procedure into one procedure, preventing misplacement of the catheter. The second is inspired by the ${ }^{166 \mathrm{Ho}}$ microsphere

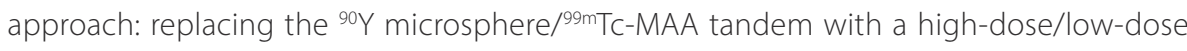
${ }^{90} \mathrm{Y}$ microsphere tandem. However, low dose ${ }^{90} \mathrm{Y}$ imaging is extremely challenging. In this thesis, the feasibility of quantitative low dose ${ }^{9} Y$ imaging was investigated. We showed that imaging by SPECT/CT is sensitive enough to accurately estimate all important parameters needed for radioembolization treatment planning. In addition, the dosimetric characteristics of different types of microspheres were compared and a the quantitative accuracy of a new digital PET/CT scanner for ${ }^{90} \mathrm{Y}$ imaging was investigated.

\section{Hybrid interventional $x$-ray and scintigraphy imaging}

With the experimental prototype of the dual layer detector evaluated in Chapter 3, we have shown that simultaneous nuclear and scintigraphy imaging with a dual layer detector is possible. However, the configuration of this prototype was not flexible and portable, and therefore not suitable for the intervention room. To take the next step towards clinical testing we needed a clinical prototype. This clinical prototype has been built and is named IXSI: Interventional X-ray and Scintigraphy Imaging (Figure 8.1). At the moment of writing, IXSI is in the process of being approved for clinical use. IXSI differs in some ways from the design discussed in Chapter 3.

First of all, the dual layer detector is mounted on a c-arm that is suited for use in the intervention room. The c-arm is mobile and can be moved in and out of the intervention room, so it only takes up space when the scanner is actually needed. Furthermore, the open design of the c-arm grants the physician easy access to the patient, even when the patient is being imaged.

Second, the c-arm can rotate around the patient to acquire projection images that can be used to reconstruct 3D images. These rotations are semi-body tracing orbits, unlike the conventional c-arms that only perform circular orbits (Figure 8.1). These semi-body tracing orbits allow to acquire nuclear images with a higher spatial resolution compared with the circular orbits.
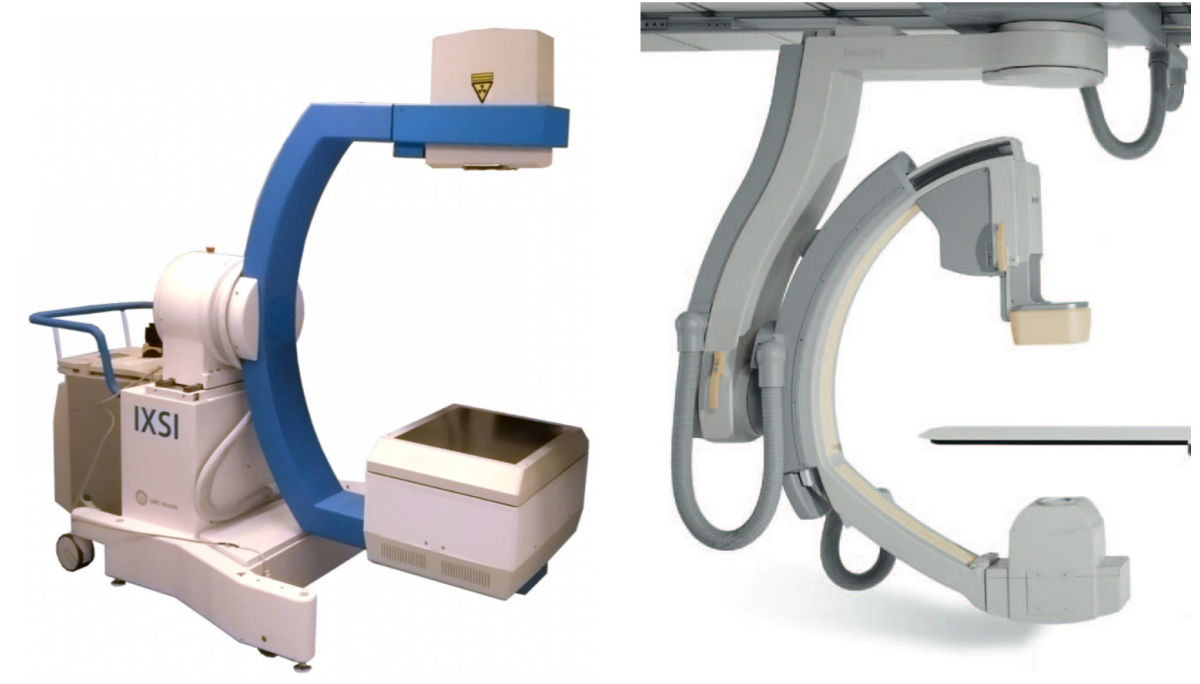

Figure 8.1: Interventional X-ray and Scintigraphy Imaging (IXSI) clinical prototype scanner (left), and a conventional c-arm system, Philips Allura Xper FD10 (right).

Third, the $x$-ray tube has been placed slightly off-focus. In the original design (Chapter 3), the $x$-ray tube was placed in the focal point of the cone beam collimator. This yields intrinsically registered fluoroscopic and nuclear images, but it also limits the peak voltage of the $x$-ray tube to $80 \mathrm{kVp}$. In a clinical setting, higher peak voltages are sedirable. By placing the $x$-ray tube slightly out of the cone beam collimator focus, fewer $x$-rays will pass the collimator and hit the gamma camera, so higher tube voltages can be used. In the current clinical prototype, the $x$-ray tube is placed $5 \mathrm{~cm}$ out of focus and can be used with peak voltages up to at least $100 \mathrm{kVp}$. The downside of this design is that $\mathrm{x}$-ray images and nuclear images are not intrinsically aligned anymore. However, this mismatch is less than $1 \mathrm{~cm}$, depending on source-detector distance, which may be acceptable given the resolution of more than $1 \mathrm{~cm}$.

\section{Clinical IXSI study}

A clinical study will be performed with IXSI in which radioembolization patients will be imaged by IXSI in the intervention room during the pre-treatment procedure. $2 \mathrm{D}$ real time images of the administration of the ${ }^{99 m} \mathrm{TC}-\mathrm{MAA}$ and $3 \mathrm{D}$ SPECT/CBCT images of the final 
dose distribution will be acquired. The clinical study will have a primary focus on the safety of the use of IXSI in the intervention room. Additional time spend in the intervention room, additional dose delivered by IXSI, ability to confirm the ${ }^{99 m T c-M A A ~ i n j e c t i o n ~ p o s i t i o n ~}$ with IXSI, and ability to perform semi-body tracing orbits, are issues that will be monitored in the study.

The secondary aim of the study is to investigate the added value of the acquired images The 2D images acquired by IXSI could provide the physician with information on the dose distribution in real time. Thus far, nuclear imaging is performed at the nuclear medicine department, after administration of ${ }^{99 m} \mathrm{mc}-M A A$, thus only showing the final dose distribution. Nuclear imaging during the administration has never been performed, and could provide valuable information about the flow dynamics of the microspheres in vivo. The physician could use this information to optimise the catheter position, for example when there is a flow of microspheres to parts that should not be treated (extrahepatic depositions, or non-target liver segments), or to add additional catheter positions when there is insufficient tumour targeting. Real-time 2D imaging would allow the physician to immediately intervene in the procedure, and to directly see the results of this intervention.

The SPECT/CBCT images acquired by IXSI can be compared with clinical SPECT/CT images acquired at the nuclear medicine department. IXSI has one detector head and will acquire a SPECT image in 10 minutes, whereas a clinical SPECT scanner has two detector heads and acquires a SPECT image in 20 minutes (current clinical protocol of a ${ }^{99 m T C-M A A ~ s c a n ~}$ at the UMC Utrecht). This means that IXSI will acquire fewer counts in the projection data compared with a clinical SPECT/CT scan. Therefore, the image quality of a SPECT image acquired by IXSI will be worse than one acquired on a clinical scanner. However, the aim of IXSI is not to provide the physician with images of the highest diagnostic quality, but with information otherwise not available. In addition, using a short acquisition time yields estimated lung shunt factions and tumour to non-tumour ratios that are as accurate as with a long acquisition time clinical protocol [173]. Furthermore, the quality of the SPECT images acquired by IXSI can be improved by motion compensation [174]. This is possible because of the simultaneous acquisition of SPECT and CBCT of IXSI. This is not possible with a clinical SPECT/CT scanner, where the SPECT and CT are acquired consecutively.

\section{Technical improvements of IXSI}

Although the clinical IXSI prototype will show to be improved over the experimental prototype discussed in Chapter 3, it can still be further optimised. Reducing the amount of aluminium surrounding the flat panel will increase the transmission of gamma rays. Furthermore, electronic components that are now positioned at the back of the flat panel could be moved to the sides. This will improve the homogeneity of the transmission.
Second, to allow for higher tube voltages to be used, one could use a scintillation crystal that has less afterglow. Examples are cerium bromide $\left(\mathrm{CeBr}_{3}\right)$ or cadmium zinc telluride (CdZnTe), but both are expensive [175]. Therefore, we chose for the more cost-effective solution of placing the $x$-ray tube off-focus, with the downside of introducing a small mismatch between nuclear and scintigraphy images.

\section{Future perspectives of IXSI}

If the physician can determine in the intervention room whether radioembolization can be performed safely, and how much activity needs to be administered, the pre-treatment procedure could be directly followed by the treatment procedure. Such a single-session, or one-day, procedure would be more time-efficient, less of a burden for the patient, and could improve the predictive value of the pre-treatment procedure. In current clinical practice, the catheter is removed before the patient is moved to the nuclear medicine department for SPECT/CT. For the treatment procedure the catheter needs to be repositioned in the exact same position as in the pre-treatment procedure. This is prone to errors, and different catheter positions often lead to differences in dose distribution, which reduces the predictive value of the pre-treatment scan [33-36]. Imaging in the intervention room with IXSI would allow the catheter to stay in place, which increases the predictive value of the pre-treatment scan.

Although IXSI is designed for radioembolization procedures, it could potentially benefit other interventional procedures as well. An example is sentinel lymph node procedures,

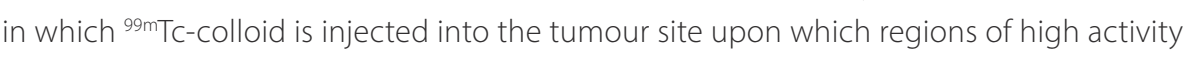
will mark the lymph nodes draining the tumour. These lymph nodes can be identified using gamma probes, or freehand SPECT [176-178]. To add anatomical information in real time, freehand SPECT can be fused with ultrasound [179]. Since IXSI is also designed to combine ${ }^{99 m} \mathrm{Tc}$ scintigraphy and anatomical imaging in real time, IXSI might improve the selection of lymph nodes

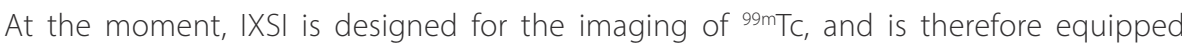
with a collimator that has similar performance as the low-energy high-resolution (LEHR) collimator. Imaging of radionuclides used for radioembolization therapy, ${ }^{90} \mathrm{Y}$ or ${ }^{166} \mathrm{Ho}$, is generally performed using high or medium energy collimators, respectively. IXSI could be altered to be equipped with a different type of collimator, to make it (more) suited for the imaging of ${ }^{90} \mathrm{Y}$ or ${ }^{166} \mathrm{Ho}$. Thereby, IXSI could be used for imaging during the treatment procedure. In this way, tumour and healthy liver dose might be monitored during administration of the microspheres. 


\section{Technical challenges of a ${ }^{90} \mathrm{Y}$ pre-treatment procedure}

In Chapters 5 and 6 , we explored the technical feasibility of the use of ${ }^{90} Y$ microspheres instead of ${ }^{99 m} \mathrm{mc}-\mathrm{MAA}$ for the pre-treatment procedure. From a technical point of view, the main challenge is imaging the extremely low activity of ${ }^{90} \mathrm{Y}$ that would be needed for a pre-treatment procedure. We showed that this is possible with SPECT/CT using a Monte Carlo-based reconstruction method. A limitation of these chapters is that the results were mainly based on phantom experiments. Only one example of a patient scan was shown. A next step would be to verify these results in patients who have different dose distributions, and to show the safety and predictive value of a ${ }^{90} \mathrm{Y}$ pre-treatment procedure in a clinical study.

During such a clinical study, new technical challenges might arise. One of those could be motion artefacts. Patients might move, and will breathe, during a SPECT/CT scan. This will lower the image quality and might impact the accuracy with which important parameters needed for radioembolization treatment planning can be determined.

Another challenge could be a reduced number of counts acquired in the projection data. The phantom experiments were performed with phantoms mimicking an average male. However, for heavier patients attenuation will be increased and this will lower sensitivity. This could again affect the accuracy of the scan, since the amount of activity that should be used for the ${ }^{90} Y$ pre-treatment procedure is already very low.

A third technical challenge is the availability of the Monte Carlo-based reconstruction method for clinical use. The accuracy of imaging the low amount of ${ }^{90} Y$ activity with SPECT depends on this reconstruction method. At the moment, the reconstruction method is only available for research purposes at the UMC Utrecht. For a clinical research study, the use of this method is will likely be acceptable. But introduction of the ${ }^{90} Y$ pre-treatment procedure in a routine clinical setting, may pose additional requirements on the Monte Carlo-based reconstruction method.

\section{Practical challenges of a ${ }^{90} \mathrm{Y}$ pre-treatment procedure}

Next to the technical challenges of a ${ }^{90} \mathrm{Y}$ pre-treatment procedure, there are also some clinical and practical challenges. A ${ }^{90} \mathrm{Y}$ pre-treatment requires a small dosage of ${ }^{90} \mathrm{Y}$ microspheres. With a one-day treatment in mind, this small dosage can be ordered together with the treatment dosage. For glass microspheres, a dosage of microspheres can be ordered as multiple vials at no additional costs, one of which could be the pretreatment dosage. For resin microspheres, the microspheres are delivered in a 'shipping vial', and the treatment dosage has to be withdrawn from this shipping vial at the treatment centre. Therefore, these microspheres can also be divided over multiple vials with the desired activity. However, when the pre-treatment procedure is performed 1-2 weeks prior to the treatment procedure, as is current clinical practice, a separate dosage of microspheres needs to be ordered. This can be quite expensive as the manufacturers of ${ }^{90} Y$ glass and ${ }^{90} Y$ resin microsphere do not offer small doses with low activity, and one would have to order an additional treatment dosage.

In Chapter 5 and 6 we argued that the total activity used for the ${ }^{90} Y$ pre-treatment procedure should be $100 \mathrm{MBq}$. It is however unclear what the ideal number of microspheres for the pre-treatment procedure would be. The ${ }^{166 \mathrm{Ho}}$ pre-treatment dosage consists of about $10 \%$ of the microspheres that are used for the treatment. In order to have the right amount of activity for the pre-treatment procedure, ${ }^{166} \mathrm{Ho}$ microspheres used for the pretreatment procedure have a lower specific activity (100 Bq/sphere) compared with the ${ }^{166} \mathrm{Ho}$ microspheres used for the treatment (240-371 Bq/sphere). Extracting the ${ }^{90} \mathrm{Y}$ pretreatment microsphere dosage from the treatment dosage, like described in the previous paragraph, will result in a pre-treatment dosage with the same specific activity as the treatment dosage. With the limit of $\sim 100 \mathrm{MBq}$ this would result in $\sim 40 \mathrm{k}$ microspheres for a ${ }^{90} \mathrm{Y}$ glass pre-treatment dosage, and $\sim 1.3 \mathrm{M}$ microspheres for a ${ }^{90} \mathrm{Y}$ resin pre-treatment dosage. It has been suggested that the number of spheres used for a (pre-)treatment procedure does influence dosimetry on a microscopic scale [134]. However, this is still a subject of research, and the ideal number of microspheres to be used needs further investigation

\section{Dose response}

Using the work described in this thesis, radioembolization procedures may be optimised and accurate dosimetry may become a reliable part of the procedure, enabling personalised treatment. Several ways to apply the quantitative images into a dose model exist, including the coarse current approach of a mono-compartment model (assuming homogeneous activity distribution), a partition model (introducing an average difference between tumorous and healthy tissue), and a voxel-based model (that includes the heterogeneous activity uptake in tumours and healthy liver, and is closest to radiotherapy models). Taking the step from the mono-compartment model to multi-compartment models or even voxel-based dosimetry for treatment planning, requires dose limits for tumours and healthy liver parenchyma [38]. The search for those dose limits is an active field of research, and is complicated because dose limits might differ between different tumour types and types of microspheres. In Chapter 4 we discussed the differences in dosimetry between the different types of microspheres for advanced intrahepatic cholangiocarcinoma. Unfortunately, the limited number of patients included in this study did not allow to define dose limits for tumour response. This requires more data, possibly gathered from multiple institutions. These multi-centre studies bring along additional 
Chapter 8. General discussion

challenges. Dose measurements require quantitative imaging, and different institutions use different imaging modalities, different scanners, and different methods to calculate dose. This makes the combination of data acquired at multiple institutions difficult [122]. Studies evaluating the performance characteristics of new scanners, and comparing them with those of their predecessor, like Chapter 7, contribute to enhancing the current literature on dosimetry in radioembolization, and are needed for multi-centre studies. For quantitative ${ }^{90} Y$ imaging using PET/CT, a wide range of scanners have been compared and recommendations regarding ideal reconstruction parameters have been made $[139,157]$. Quantitative ${ }^{90} Y$ imaging using SPECT/CT often relies on Monte Carlo-based reconstruction methods $[17,18,161]$. Efforts need to be made to share these reconstruction algorithms and to compare their performances across scanner and patient characteristics. 


\section{Chapter 9}

\section{Summary en Nederlandse}

samenvatting 


\section{Summary}

Radioembolization is a minimally invasive treatment for liver cancer. During a radioembolization procedure small radioactive spheres (microspheres) are administered in the vasculature of the liver. These microspheres lodge (mostly) in the tumorous tissue, where they locally irradiate liver tumours, whilst (mostly) sparing the healthy liver tissue. Prior to the treatment procedure, a pre-treatment safety procedure is performed in which surrogate particles, technetium-99m labelled macroaggregated albumin ( ${ }^{99 m \mathrm{~m} T-M A A)}$, are administered to simulate the distribution of the microspheres. These ${ }^{99 \mathrm{mTC}} \mathrm{MAA}$ particles are visualised using nuclear imaging. Based on the amount of ${ }^{99 m T c-M A A}$ shunting to the lungs (lung shunt fraction), the unwanted accumulation of ${ }^{99 m} \mathrm{Tc}-\mathrm{MAA}$ elsewhere outside the liver (extrahepatic depositions), and the distribution of 99mTc-MAA within the liver (intrahepatic dose distribution), a nuclear medicine physician decides whether or not it is safe to proceed with the treatment procedure. When considered safe, the treatment will usually take place $1-2$ weeks later.

The microspheres that are administered during the radioembolization treatment have a certain level of radioactivity, measured in becquerel (Bq). Deciding where and how much activity needs to be administered for treatment is called treatment planning. The calculation of the amount of activity that needs to be administered is based on physical models, but these are generally not tailored to individual patients. This thesis introduced several ways to improve radioembolization treatment planning using nuclear imaging.

Since radioembolization is a minimally invasive procedure, the physician relies on medical imaging to look inside the patient. The current status of technical advances in image guidance of radionuclide therapy is discussed in Chapter 2. Traditional imaging modalities used to guide radionuclide therapies are the nuclear imaging modalities single photon emission computed tomography (SPECT) and positron emission tomography (PET). Both modalities are able to produce three dimensional images of the radionuclide distribution, by solving a mathematical problem referred to as image reconstruction.

One of the advances in SPECT is to include Monte Carlo simulations into the image reconstruction algorithm. These simulations model the behaviour of individual photons in detail. By doing SO, SPECT can produce quantitative images. This means that an image not only tells you where radionuclides are located (qualitative image), but also quantifies how much activity in $\mathrm{Bq} / \mathrm{mL}$ there is (quantitative image).
One of the advances in PET is the introduction of a new detector type, so called silicon photomultipliers. This results in a PET scanner with improved spatial and temporal resolution, which both contribute to better image quality.

Chapter 2 also discusses a new imaging modality that is able to simultaneously acquire $x$-ray and nuclear images in the intervention room, which is something that cannot be done by any of the current available scanners. This can be achieved by equipping a conventional c-arm (a scanner currently used in the intervention room to acquire x-ray images) with a gamma camera (the detector part of a SPECT scanner). Straightforward combination of these elements will block the line of view of one of them. Therefore, a design is proposed where the gamma camera can look around the $x$-ray tube. Such a design is feasible, and could provide the physician with valuable nuclear information in the intervention room during procedures like radioembolization.

In Chapter $\mathbf{3}$ we introduced a new design for the interventional scanner that combines $x$-ray and nuclear imaging. In the old design, where the gamma camera looks around the $x$-ray tube, an intermediate reconstruction step is needed in order to produce overlaying $x$-ray and nuclear images. In the new design we introduced a dual layer detector, where the gamma camera is placed behind the $x$-ray detector. Although in this design the line of view of the gamma camera is blocked by the $x$-ray detector, the $x$-ray detector turned out to be transparent enough for the gamma camera to acquire nuclear images. The advantage of this design is that the x-ray and nuclear images are intrinsically registered, which makes the intermediate reconstruction step redundant. In this chapter we build and characterised a prototype dual layer scanner. With this scanner we are able to acquire real-time intrinsically registered $\mathrm{x}$-ray and nuclear images, albeit at the cost of reduced sensitivity for the nuclear images.

In Chapter $\mathbf{4}$ we compared the three different types of microspheres that are used for radioembolization. Initially, radioembolization was performed with the radionuclide yttrium-90 $\left({ }^{90} \mathrm{Y}\right)$. There are ${ }^{90} \mathrm{Y}$ glass and ${ }^{90} \mathrm{Y}$ resin microspheres commercially available. More recently, microspheres labelled with holmium-166 (166 Ho) became commercially available. In this chapter we used quantitative ${ }^{90} \mathrm{Y} \mathrm{PET/CT}$ and quantitative ${ }^{166} \mathrm{Ho} \mathrm{SPECT/CT}$ to compare the delivered dose in advanced intrahepatic cholangiocarcinoma patients. We show that the ${ }^{90} \mathrm{Y}$ glass microspheres delivered the highest tumour dose, whilst the overall healthy liver dose did not significantly differ from the other microspheres. In addition, we compared the response to treatment and the toxicity between these groups. Although the three microspheres have different physical characteristics and show differences in dosimetry, toxicity and response were similar for all groups. This 
emphasis the need for separate dose constraints and dose targets to be used for treatment planning for each particle.

In Chapter $\mathbf{5}$ we tried to improve the lung shunt fraction estimation. We know that this estimate is inaccurate when based on a ${ }^{99 \mathrm{~m} T \mathrm{C}-M A A}$ pre-treatment safety procedure. The MAA particles differ from the microspheres, and therefore lead to differences in lung shunt fraction. Using the same ${ }^{90} Y$ labelled microspheres for the pre-treatment safety procedure, instead of the MAA particles, could solve this problem. However, to ensure that the pretreatment procedure can be performed safely, even when a patient turns out to have an unfavourable dose distribution, the activity of the ${ }^{90} Y$ needs to be kept very low $(\sim 100 \mathrm{MBq})$. This leads to problems regarding the imaging of the ${ }^{90} \mathrm{Y}$. Imaging of ${ }^{90} \mathrm{Y}$ can be performed with PET/CT and SPECT/CT, and in this chapter we investigated whether these modalities were suitable for the estimation of the lung shunt fraction using a low activity of ${ }^{90}$. This was done using a phantom (a testing dummy that mimics a human), which allows us to control the true lung shunt fraction. This study showed that we can accurately estimate the lung shunt fraction using a low activity of ${ }^{90} \mathrm{Y}$, as long as we use SPECT/CT with dedicated Monte Carlo-based reconstruction software for imaging

In Chapter $\mathbf{6}$ we investigated whether we could also accurately image extrahepatic depositions and intrahepatic dose distributions at a low activity of ${ }^{90}$ Y. Again, we used a phantom to show that SPECT/CT is capable of accurate imaging at this low activity, whereas $\mathrm{PET} / \mathrm{CT}$ unfortunately is not. In addition we simulated a ${ }^{90} \mathrm{Y}$ pre-treatment procedure in a radioembolization patient by acquiring a very fast SPET/CT scan to mimic the low activity of ${ }^{\circ}$ Y. This showed that even in a patient, who does not have a uniform dose distribution like the phantom, SPECT/CT can accurately image this distribution at a low ${ }^{90} Y$ activity.

In Chapter $\mathbf{7}$ we characterised a new PET/CT scanner with silicon photomultipliers for ${ }^{90} Y$ imaging. This new PET/CT scanner has improved resolution and sensitivity compared with the PET/CT scanner that was used for the ${ }^{90} Y$ experiments described in Chapter 5 and 6. We therefore hoped that this new PET/CT would be more suited for imaging ${ }^{90} \mathrm{Y}$ at low activities. However, although the new PET/CT improved image quality (lower noise level), it did not improve quantification of ${ }^{90} \mathrm{Y}$. Therefore, this new PET/CT scanner is not suitable for imaging the low ${ }^{90} Y$ activity that would be needed for a ${ }^{90} Y$ pre-treatment procedure.

In this thesis two improvements for radioembolization treatment planning were proposed The first is the introduction of a dual layer scanner for simultaneous $x$-ray and nuclear imaging in the intervention room. Apart from the experimental prototype that has been built, a clinical prototype is being built to show whether this system can be used safely to guide radioembolization procedures. The second is the proposal of a ${ }^{90} \mathrm{Y}$ pre-treatment procedure to increase the predictive power of the pre-treatment procedure. We showed that parameters needed for radioembolization treatment planning can be accurately estimated when acquired by SPECT/CT. 


\section{Nederlandse samenvatting}

Radioembolisatie is een minimaal invasieve behandeling van lever kanker. Tijdens een radioembolistie behandeling worden er radioactief gelabelde bolletjes (microsferen) toegediend in het vaatstelsel van de lever. Deze microsferen lopen (vooral) vast in het tumorweefsel, waar ze de lever tumoren lokaal bestralen, terwijl het gezonde leverweefsel (grotendeels) gespaard blijft. Voorafgaand aan de behandeling wordt een veiligheidsprocedure uitgevoerd, waarin soortgelijke deeltjes, technetium-99m macro

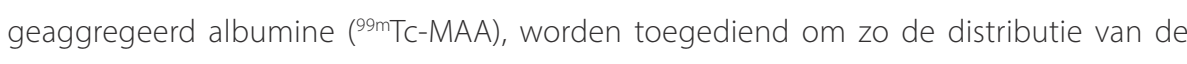
microsferen na te bootsen. Deze ${ }^{99 m}$ Tc-MAA deeltjes worden zichtbaar gemaakt door middel van nucleaire beeldvorming. Op basis van de hoeveelheid ${ }^{99 m}$ Tc-MAA die in de longen terecht komt (long shunt fractie), de ongewenste verzameling van ${ }^{99 m} \mathrm{mc}-\mathrm{MAA}$ ergens anders buiten de lever (extra hepatische deposities), en de verdeling van ${ }^{99 m} \mathrm{mc}-\mathrm{MAA}$ binnen de lever (intra hepatische dosis verdeling), besluit een nucleair geneeskundige of het veilig is om met de behandeling door te gaan. Wanneer dit het geval is vindt de behandeling meestal 1-2 weken later plaats.

De microsferen die worden toegediend tijdens een radioembolisatie behandeling hebben een bepaalde hoeveelheid radioactiviteit, gemeten in becquerel (Bq). Het bepalen van waar en hoeveel activiteit er toegediend moet worden tijdens de behandeling wordt ook wel behandelplanning genoemd. De berekening van de hoeveelheid activiteit die toegediend moet worden is gebaseerd op natuurkundige modellen, maar deze worden over het algemeen niet op maat gemaakt voor individuele patiënten. Dit proefschrift introduceert verschillende methoden om de behandelplanning van radioembolisatie te verbeteren met behulp van nucleaire beeldvorming.

Omdat radioembolisatie een minimaal invasieve behandeling is, is de arts afhankelijk van medische beeldvorming om in de patiënt te kunnen kijken. De huidige status van technische ontwikkelingen in medische beeld begeleiding bij radionuclidentherapie wordt besproken in Hoofdstuk 2. Traditionele beeldvormende technieken gebruikt bij radionuclidentherapieën zijn de nucleaire beeldvormende technieken single photon emission computed tomography (SPECT) en positron emission tomography (PET). Beide technieken kunnen drie dimensionele plaatjes maken van de radionuclide verdeling, door een wiskundig probleem op te lossen wat ook wel beeldreconstructie wordt genoemd.

Een van de vooruitgangen bij SPECT is de introductie van Monte Carlo simulaties in de beeldreconstructie. Deze simulaties simuleren het gedrag van individuele fotonen in detail. Door dit te doen kunnen er met SPECT kwantitatieve beelden verkregen worden. Dit betekent dat een beeld niet alleen informatie geeft over waar radionucliden zich bevinden (kwalitatieve beelden), maar ook kan kwantificeren hoeveel activiteit in $\mathrm{Bg} / \mathrm{mL}$ er is (kwantitatieve beelden).

Een van de vooruitgangen bij PET is de introductie van een nieuw detector type, de silicon photomultipliers. Dit zorgt voor een PET scanner met een verbeterde spatiele en temporele resolutie, die beide de beeld kwaliteit verhogen.

Hoofdstuk 2 beschrijft ook een nieuwe beeldvormende techniek die nucleaire en röntgen beelden tegelijkertijd kan verkrijgen in de behandel kamer, iets wat niet gedaan kan worden door de scanners die momenteel beschikbaar zijn. Dit kan gedaan worden door een standaard c-arm (een scanner die momenteel wordt gebruikt in de behandelkamer om röntgen beelden te maken) uit te rusten met een gamma camera (het detector deel van een SPECT scanner). Het eenvoudig combineren van deze twee elementen zal het gezichtsveld van een van de twee blokkeren. Daarom wordt een ontwerp voorgesteld waarin de gamma camera om de röntgenbuis heen kijkt. Dit ontwerp is uitvoerbaar en kan de arts waardevolle informatie geven in de behandelkamer tijdens behandelingen zoals radioembolisatie.

In Hoofdstuk 3 beschrijven we een nieuw ontwerp voor de interventionele scanner die nucleaire en röntgen beelden kan verkrijgen. In het oude ontwerp, waar de gamma camera om de röntgenbuis heen kan kijken, was een tussenliggende beeldreconstructie stap nodig om overlappende nucleaire en röntgen beelden te verkrijgen. In het nieuwe ontwerp introduceren we een dubbellaags detector, waar de gamma camera achter de röntgen detector zit. Ook al is het gezichtsveld van de gamma camera geblokkeerd door de röntgen detector, de röntgen detector blijkt doorzichtig genoeg te zijn voor de gamma camera om nucleaire beelden te verkrijgen. Het voordeel van dit ontwerp is dat de nucleaire en röntgen beelden automatisch overlappen, wat de tussenliggende beeldreconstructie stap overbodig maakt. In dit hoofdstuk hebben we een prototype dubbellaags detector scanner gemaakt en gekarakteriseerd. Met deze scanner kunnen we automatisch overlappende nucleaire en röntgen beelden live verkrijgen, al is het tegen de prijs van een verminderde sensitiviteit voor de nucleaire beelden.

In Hoofdstuk 4 vergeleken we de drie verschillende type microsferen die gebruikt worden voor radioembolisatie. Aanvankelijk werd bij radioembolisatie gebruik gemaakt van het radionuclide yttrium-90 ( $\left.{ }^{90} \mathrm{Y}\right)$. Er zijn ${ }^{90} \mathrm{Y}$ glas en ${ }^{90} \mathrm{Y}$ hars microsferen commercieel verkrijgbaar. Recentelijk zijn er ook microsferen beschikbaar gekomen die gelabeld zijn met holmium-166 (166 Ho). In dit hoofdstuk hebben we kwantitatieve ${ }^{90} \mathrm{Y} \mathrm{PET/CT} \mathrm{en}$

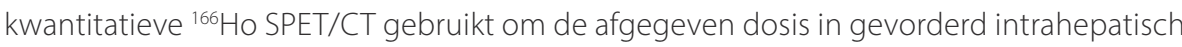
cholangiocarcinoom te vergelijken. We hebben laten zien dat de ${ }^{90} \mathrm{Y}$ glas microsferen de 
hoogste dosis op de tumor geven, terwijl de dosis op het algehele gezonde leverweefsel niet significant verschilde tussen de microsferen. In aanvulling daarop hebben we de reactie op de behandeling en de toxiciteit tussen deze groepen vergeleken. Hoewel de drie typen microsferen verschillende fysieke karakteristieken hebben en ze verschillen in geabsorbeerde dosis laten zien, was de reactie op behandeling en de toxiciteit gelijkwaardig tussen de groepen. Dit benadrukt de noodzaak van afzonderlijke dosisbeperkingen en dosisdoelen die moeten worden gebruikt voor de behandelplanning voor elk type microsfeer.

In Hoofdstuk 5 hebben we geprobeerd de long shunt fractie schatting te verbeteren. We weten dat deze schatting onnauwkeurig is als deze gebaseerd is op de ${ }^{99 m T C}$ MAA veiligheidsprocedure. De MAA deeltjes verschillen namelijk van de microsferen, wat tot een verschil in long shunt fractie leidt. Door dezelfde ${ }^{90} Y$ microsferen voor de veiligheidsprocedure te gebruiken, in plaats van de MAA deeltjes, zou dit probleem opgelost kunnen worden. Maar, om er zeker van te zijn dat de veiligheidsprocedure veilig uitgevoerd kan worden, ook als de patiënt een ongunstige dosis verdeling blijkt te hebben, moet de ${ }^{90}$ Y activiteit heel erg laag blijven ( 100 MBq). Dit zorgt voor problemen bij de beeldvorming van ${ }^{90} \mathrm{Y}$. De beeldvorming van ${ }^{90} \mathrm{Y}$ kan gedaan worden met PET/CT en met SPET/CT, en in dit hoofdstuk hebben we gekeken of deze modaliteiten geschikt zijn om de long shunt fractie te schatten bij een lage ${ }^{90} Y$ activiteit. Hiervoor hebben we gebruik gemaakt van een fantoom (een dummy die een mens nabootst), zodat we de werkelijke long shunt fractie konden controleren. Hiermee hebben we laten zien dat we de long shunt fractie accuraat kunnen schatten bij een lage ${ }^{90} \mathrm{Y}$ activiteit, zolang we SPECT/CT met Monte Carlo simulaties in de beeldreconstructie gebruiken voor de beeldvorming.

In Hoofdstuk 6 onderzochten we of we ook de extrahepatisch deposities en de intrahepatisch dosis verdeling nauwkeurig in beeld konden brengen bij een lage ${ }^{90} Y$ activiteit. Wederom hebben we gebruik gemaakt van een fantoom om te laten zien dat dit mogelijk is met SPET/CT, terwijl dit met PET/CT niet het geval is. In aanvulling hierop hebben we een ${ }^{90} Y$ veiligheidsprocedure in een radioembolisatie patiënt gesimuleerd door een hele snelle SPECT/CT scan op te nemen die zo een lage ${ }^{90} Y$ activiteit na bootst. Dit liet zien dat ook bij een patiënt, die niet uniforme dosis verdeling heeft zoals het fantoom, SPECT/CT nauwkeurig de dosis verdeling van een lage activiteit ${ }^{90} \mathrm{Y}$ kan afbeelden.

In Hoofdstuk 7 hebben we een nieuwe PET/CT scanner met silicon photomultipliers gekarakteriseerd voor het afbeelden van ${ }^{90} \mathrm{Y}$. Deze nieuwe PET/CT scanner heeft een verbeterde resolutie en sensitiviteit vergeleken met de PET/CT scanner die was gebruikt voor de ${ }^{90} Y$ experimenten beschreven in hoofdstuk 5 en 6 . Daarom hoopten we dat deze nieuwe PET/CT scanner geschikter zou zijn voor het afbeelden van lage activiteiten ${ }^{90} \mathrm{Y}$.
Maar, ondanks dat de nieuwe PET/CT scanner een betere beeld kwaliteit oplevert (minder ruis), verbetert het niet de kwantificatie van ${ }^{90} \mathrm{Y}$. Daarom is deze nieuwe PET/CT scanner niet geschikt voor het afbeelden van een lage activiteit ${ }^{90} \mathrm{Y}$ die nodig is voor een ${ }^{90} \mathrm{Y}$ veiligheidsprocedure.

In dit proefschrift zijn twee verbeteringen voor radioembolisatie therapie planning besproken. De eerste is een dubbellaags detector voor het tegelijkertijd maken van nucleaire en röntgen beelden in de behandelkamer. Behalve dat er een experimenteel prototype is gebouwd, is en ook een klinisch prototype gebouwd om te laten zien of dit systeem veilig gebruikt kan worden voor radioembolisatie procedures. De tweede is het voorstel van een ${ }^{90} \mathrm{Y}$ veiligheidsprocedure om de voorspellende waarde van de veiligheidsprocedure te vergroten. We lieten zien dat de parameters die nodig zijn voor de behandelplanning van radioembolisatie nauwkeurig kunnen worden geschat als deze gemeten worden op met behulp van SPECT/CT. 


\section{References}

1. Van Audenhaege K, Van Holen R, Vandenberghe S, Vanhove C, Metzler SD, Moore SC. Review of SPECT collimator selection, optimization, and fabrication for clinical and preclinical imaging. Med Phys. 2015;42:4796-813.

2. Horger M, Bares R. The role of single-photon emission computed tomography/computed tomography in benign and malignant bone disease. Semin Nucl Med. United States; 2006;36:28694.

3. Paul AK, Nabi HA. Gated myocardial perfusion SPECT: basic principles, technical aspects, and clinical applications. J Nucl Med Technol. United States; 2004;32:179.

4. Giammarile F, Alazraki N, Aarsvold JN, Audisio RA, Glass E, Grant SF, et al. The EANM and SNMM practice guideline for lymphoscintigraphy and sentinel node localization in breast cancer. Eur J NuC Med Mol Imaging. Germany; 2013;40:1932-47.

5. Vandenberghe S, Mikhaylova E, D'Hoe E, Mollet P, Karp JS. Recent developments in time-of-flight PET. EJNMMMI Phys. 2016/02/16. Springer International Publishing: 2016:3:3.

6. Vandenberghe $S$, D'Asseler $Y$, Van de Walle R, Kauppinen $T$, Koole M, Bouwens $L$, et al. Iterative reconstruction algorithms in nuclear medicine. Comput Med Imaging Graph. 2001;25:105-11.

7. Willowson K, Bailey DL, Baldock C. Quantitative SPECT reconstruction using CT-derived corrections. Phys Med Biol. IOP Publishing; 2008;53:3099-112.

8. Kalantari F, Rajabi H, Saghari M, Ardekani AE. Assessment of the effect of 3D resolution recovery during SPECT image reconstruction on quantification of small liver tumors: A simulation study. 201 IEEE Nucl Sci Symp Conf Rec. 2011. p. 2891-4.

9. Jaszczak RJ, Greer KL, Floyd CEJ, Harris CC, Coleman RE. Improved SPECT quantification using compensation for scattered photons. J Nucl Med. United States; 1984;25:893-900.

10. Polycarpou I, Thielemans K, Manjeshwar R, Aguiar P, Marsden PK, Tsoumpas C. Comparative evaluation of scatter correction in 3D PET using different scatter-level approximations. Ann Nuc Med. Japan; 2011;25:643-9.

11. Brasse D, Kinahan PE, Lartizien C, Comtat C, Casey M, Michel C. Correction methods for random coincidences in fully 3D whole-body PET: impact on data and image quality. J Nucl Med. 2005;46:859-67.

12. De Jong HWAM, Slijpen TP, Beekman FJ. Acceleration of Monte Carlo SPECT simulation using convolution-based forced detection. Nucl Sci IEEE Trans. 2001:48:58-64.

13. Beekman FJ, De Jong HW a M, Van Geloven S. Efficient fully 3-D iterative SPECT reconstruction with Monte Carlo-based scatter compensation. IEEE Trans Med Imaging. 2002;21:867-77.

14. Xiao J, de Wit TC, Staelens SG, Beekman FJ. Evaluation of 3D Monte Carlo-based scatter correction for 99mTc cardiac perfusion SPECT. J Nucl Med. 2006;47:1662-9.

15. Siman W, Mikell JK, Kappadath SC. Practical reconstruction protocol for quantitative90Y bremsstrahlung SPECT/CT. Med Phys. 2016;43:5093-103.

16. Elschot M, Nijsen JFW, Dam AJ, de Jong HWAM. Quantitative evaluation of scintillation camera imaging characteristics of isotopes used in liver radioembolization. PLoS One. 2011;6.

17. Elschot M, Smits MLJ, Nijsen JFW, Lam MGEH, Zonnenberg BA, van den Bosch MAAJ, et al. Quantitative Monte Carlo-based 90Y SPECT reconstruction. J Nucl Med. 2013;54:1557-63.

18. Dewaraja YK, Chun SY, Srinivasa RN, Kaza RK, Cuneo KC, Majdalany BS, et al. Improved quantitative 90Y bremsstrahlung SPECT/CT reconstruction with Monte Carlo scatter modeling: Med Phys. 2017:44:6364-76.

19. Lhommel R, Goffette P, Van den Eynde M, Jamar F, Pauwels S, Bilbao Jl, et al. Yttrium-90 TOF PET scan demonstrates high-resolution biodistribution after liver SIRT. Eur I Nucl Med Mol Imaging 2009;36:1696
20. Nickles RJ, Roberts AD, Nye JA, Converse AK, Barnhart TE, Avila-Rodriguez MA, et al. Assaying and PET imaging of Ytrrium-90: 1-34ppm>0. IEEE Nucl Sci Symp Conf Rec. IEEE; 2004. p. 3412-4.

21. Burrill J, Hafeli U, M Liu D. Advances in Radioembolization - Embolics and Isotopes. J Nucl Med Radiat Ther. 2011;01.

22. Westcott MA, Coldwell DM, Liu DM, Zikria JF. The development, commercialization, and clinical context of yttrium-90 radiolabeled resin and glass microspheres. Adv Radiat Oncol. Elsevier; 2016;1:351-64.

23. Sirtex Medical. SIRSphere Y90 Insert Instructions For Use [Internet]. 2017. Available from: https:// www.sirtex.com/media/155126/ssl-us-13.pdf

24. Quirem Medical BV Instructions for Use QuiremSpheres [Internet]. 2018. Available from: http $/ /$ www.quirem.com/wp-content/uploads/delightful-downloads/2019/02/LS-1101-10_04-IFUQuiremSpheres-Multilanguage.pdf

25. BTG. TheraSphere ${ }^{\mathrm{TM}}$ Yttrium-90 Glass Microspheres - Instructions for Use. 2015.

26. Smits MLJ, Nijsen JFW, van den Bosch MAAJ, Lam MGEH, Vente MAD, Mali WPTM, et al. Holmium-166 radioembolisation in patients with unresectable, chemorefractory liver metastases (HEPAR trial): A phase 1, dose-escalation study. Lancet Oncol. 2012;13:1025-34.

27. Houle S, Yip TK, Shepherd FA, Rotstein LE, Sniderman KW, Theis E, et al. Hepatocellular carcinoma: pilot trial of treatment with Y-90 microspheres. Radiology. United States; 1989;172:857-60.

28. Andrews JC, Walker SC, Ackermann RJ, Cotton LA, Ensminger WD, Shapiro B. Hepatic Radioembolization with Yttrium-90 Containing Glass Microspheres: Preliminary Results and Clinical Follow-Up. J Nucl Med. 1994;35:1637-44.

29. Gulec SA, Mesoloras G, Stabin M. Dosimetric Techniques in 90Y-Microsphere Therapy of Liver Cancer: The MIRD Equations for Dose Calculations. J Nucl Med. 2006;47:1209-11.

30. Vente M a D, Nijsen JFW, De Wit TC, Seppenwoolde JH, Krijger GC, Seevinck PR, et al. Clinical effects of transcatheter hepatic arterial embolization with holmium-166 poly(L-lactic acid) microspheres in healthy pigs. Eur J Nucl Med Mol Imaging. 2008;35:1259-71

31. Kennedy A, Nag S, Salem R, Murthy R, McEwan AJ, Nutting C, et al. Recommendations for Radioembolization of Hepatic Malignancies Using Yttrium-90 Microsphere Brachytherapy: A Consensus Panel Report from the Radioembolization Brachytherapy Oncology Consortium. Int J Radiat Oncol. 2007;68:13-23.

32. Beijst C, Elschot M, Viergever MA, de Jong HWAM. Toward Simultaneous Real-Time Fluoroscopic and Nuclear Imaging in the Intervention Room. Radiology. 2016;278:232-8.

33. Kafrouni M, Allimant C, Fourcade M, Vauclin S, Guiu B, Mariano-Goulart D, et al. Analysis of differences between (99m)TC-MAA SPECT- and (90)Y-microsphere PET-based dosimetry for hepatocellular carcinoma selective internal radiation therapy. EJNMMI Res. 2019:9:62.

34. Wondergem M, Smits MLJ, Elschot M, de Jong HWAM, Verkooiien HM, van den Bosch MAAJ, et al. 99mTc-Macroaggregated Albumin Poorly Predicts the Intrahepatic Distribution of 90Y Resin Microspheres in Hepatic Radioembolization. J Nucl Med. 2013;54:1294-301.

35. Gnesin S, Canetti L, Adib S, Cherbuin N, Silva Monteiro M, Bize P, et al. Partition Model-Based 99mTcMAA SPECT/CT Predictive Dosimetry Compared with 90Y TOF PET/CT Posttreatment Dosimetry in Radioembolization of Hepatocellular Carcinoma: A Quantitative Agreement Comparison. J Nuc Med. 2016:57:1672-8.

36. Richetta E, Pasquino M, Poli M, Cutaia C, Valero C, Tabone M, et al. PET-CT post therapy dosimetry in radioembolization with resin (90)Y microspheres: Comparison with pre-treatment SPECT-CT (99m) TC-MAA results. Phys Med. Italy; 2019;64:16-23.

37. Braat AJAT, Smits MLJ, Braat MNGJA, van den Hoven AF, Prince JF, de Jong HWAM, et al. ${ }^{90}$ Y Hepatic Radioembolization: An Update on Current Practice and Recent Developments. J Nucl Med. 2015;56:1079-87. 
38. Smits ML, Elschot M, Sze DY, Kao YH, Nijsen JFW, lagaru AH, et al. Radioembolization Dosimetry: The Road Ahead. Cardiovasc Intervent Radiol. 2015;38:261-9.

39. Elschot M, Nijsen JFW, Lam MGEH, Smits MLJ, Prince JF, Viergever MA, et al. $99 \mathrm{mTC}$-MAA overestimate the absorbed dose to the lungs in radioembolization: a quantitative evaluation in patients treated with ${ }^{166} \mathrm{Ho}$-microspheres. Eur J Nucl Med Mol Imaging. 2014;41:1965-75.

40. Yu N, Srinivas SM, Difilippo FP, Shrikanthan S, Levitin A, McLennan G, et al. Lung dose calculation with SPECT/CT for 90Yittrium radioembolization of liver cancer. Int J Radiat Oncol Biol Phys. Elsevier Inc: 2013;85:834-9.

41. Lopez B, Mahvash A, Lam MGEH, Kappadath SC. Calculation of lung mean dose and quantification of error for 90Y-microsphere radioembolization using 99mTc-MAA SPECT/CT and diagnostic chest CT. Med Phys. 2019;0,

42. Hung JC, Redfern MG, Mahoney DW, Thorson LM, Wiseman GA. Evaluation of Macroaggregated Albumin Particle Sizes for Use in Pulmonary Shunt Patient Studies. J Am Pharm Assoc. Elsevier 2000;40:46-51.

43. De Gersem R, Maleux G, Vanbilloen H, Baete K, Verslype C, Haustermans K, et al. Influence of Time Delay on the Estimated Lung Shunt Fraction on 99mTc-Labeled MAA Scintigraphy for 90Y Microsphere Treatment Planning. Clin Nucl Med. 2013;38.

44. Lambert B, Mertens J, Sturm EJ, Stienaers S, Defreyne L, D'Asseler Y. 99mTc-labelled macroaggregated albumin (MAA) scintigraphy for planning treatment with 90Y microspheres. Eur J Nucl Med Mol Imaging. 2010;37:2328-33.

45. Kafrouni M, Allimant C, Fourcade M, Vauclin S, Delicque J, llonca A-D, et al. Retrospective Voxel-Based Dosimetry for Assessing the Ability of the Body-Surface-Area Model to Predict Delivered Dose and Radioembolization Outcome. J Nucl Med. 2018;59:1289-95,

46. Jadoul A, Bernard C, Lovinfosse P, Gérard L, Lilet H, Cornet O, et al. Comparative dosimetry between (99m)TC-MAA SPECT/CT and (90)Y PET/CT in primary and metastatic liver tumors. Eur J Nucl Med Mol Imaging. Germany; 2020;47:828-37.

47. Smits ML, Dassen MG, Prince JF, Braat AJAT, Beijst C, Bruijnen RCG, et al. The superior predictive value of $166 \mathrm{Ho}-$ scout compared with 99mTc-macroaggregated albumin prior to $166 \mathrm{Ho}$-microspheres radioembolization in patients with liver metastases. Eur J Nucl Med Mol Imaging. 2019;

48. Kwekkeboom DJ, de Herder WW, Kam BL, van Eijck CH, van Essen M, Kooij PP, et al. Treatment with the radiolabeled somatostatin analog [177 Lu-DOTA 0,Tyr3]octreotate: toxicity, efficacy, and survival. J Clin Oncol. 2008;26:2124-30.

49. Seidlin SM, Marinelli LD, Oshry E. Radioactive iodine therapy; effect on functioning metastases of adenocarcinoma of the thyroid. J Am Med Assoc. 1946;132:838-47.

50. Smits MLJ, Prince JF, Rosenbaum CENM, van den Hoven AF, Nijsen JFW, Zonnenberg BA, et al. Intraarterial radioembolization of breast cancer liver metastases: a structured review. Eur J Pharmacol. 2013;709:37-42.

51. Ogawa K, Harata Y, Ichihara T, Kubo A, Hashimoto S. A practical method for position-dependent Compton-scatter correction in single photon emission CT. IEEE Trans Med Imaging. IEEE, 1991;10:408-12.

52. Zeng GL, Gullberg GT, Tsui BMW, Terry JA. Three-dimensional iterative reconstruction algorithms with attenuation and geometric point response correction. IEEE Trans Nucl Sci. 1991:38.693-702.

3. Walrand $\mathrm{S}$, Hesse M, Wojcik R, Lhommel R, Jamar F. Optimal design of anger camera for bremsstrahlung imaging: Monte Carlo evaluation. Front Oncol. 2014;4:149,

54. Schramm NU, Ebel G, Engeland U, Schurrat T, Behe M, Behr TM. High-resolution SPECT using multipinhole collimation. Nucl Sci IEEE Trans. 2003;50:315-20.

55. Beijst C, Elschot M, Viergever MA, de Jong HWAM. A parallel-cone collimator for high-energy SPECT. J Nucl Med. 2015;56:476-82.
56. Walrand S, Hesse M, Demonceau G, Pauwels S, Jamar F. Yttrium-90-labeled microsphere tracking during liver selective internal radiotherapy by bremsstrahlung pinhole SPECT: feasibility study and evaluation in an abdominal phantom. EJNMMI Res. 2011;1:32.

57. Sharir T, Slomka PJ, Berman DS. Solid-state SPECT technology: fast and furious. J Nucl Cardiol. 2010;17:890-6.

58. Melis M, de Swart J, de Visser M, Berndsen SC, Koelewijn S, Valkema R, et al. Dynamic and static smallanimal SPECT in rats for monitoring renal function after 177Lu-labeled Tyr3-octreotate radionuclide therapy. J Nucl Med. Society of Nuclear Medicine; 2010;51:1962-8.

59. Kojima A, Gotoh K, Shimamoto M, Hasegawa K, Okada S. Iodine-131 imaging using 284 keV photons with a small animal CZT-SPECT system dedicated to low-medium-energy photon detection. Ann Nucl Med. 2016;30:169-75.

60. van Gils CAJJ, Beijst C, Van Rooij R, de Jong HWAMAM. Impact of reconstruction parameters on quantitative I-131 SPECT. Phys Med Biol. IOP Publishing; 2016;61:5166-82.

61. Elschot M, Smits ML, Nijsen JFW, Lam MGEH, Zonnenberg B a, van den Bosch M a a J, et al. Quantitative Monte Carlo-based holmium-166 SPECT reconstruction. Med Phys. 2013:40:11250212.

62. Bailey DL, Willowson KP. Quantitative SPECT/CT: SPECT joins PET as a quantitative imaging modality. Eur J Nucl Med Mol Imaging. 2014:41 Suppl 1:S17-25.

63. Dewaraja YK, Frey EC, Sgouros G, Brill a. B, Roberson P. Zanzonico PB, et al. MIRD pamphlet no. 23 Quantitative SPECT for patient-specific 3-dimensional dosimetry in internal radionuclide therapy. J Nucl Med. 2012;53:1310-25.

64. Akabani G, Hawkins WG, Eckblade MB, Leichner PK. Patient-specific dosimetry using quantitative SPECT imaging and three-dimensional discrete Fourier transform convolution. J Nucl Med. 1997;38:308-14.

65. Lehovich A, Bruyant PP, Gifford HS, Schneider PB, Squires S, Licho R, et al. Impact on reader performance for lesion-detection/ localization tasks of anatomical priors in SPECT reconstruction. IEEE Trans Med Imaging. NIH Public Access; 2009;28:1459-67.

66. Reader AJ, Verhaeghe J. 4D image reconstruction for emission tomography. Phys Med Biol. 2014;59:R371-418

67. Zaidi H, Montandon M-L. Scatter Compensation Techniques in PET. PET Clin. 2007;2:219-34.

68. Casey ME, Hoffman EJ. Quantitation in positron emission computed tomography: 7. A technique to reduce noise in accidental coincidence measurements and coincidence efficiency calibration. I Comput Assist Tomogr. 10:845-50

69. Slomka PJ, Pan T, Germano G. Recent Advances and Future Progress in PET Instrumentation. Semin Nucl Med. Elsevier; 2016;46:5-19.

70. Surti S. Update on time-of-flight PET imaging. J Nucl Med. 2015;56:98-105.

71. Conti M. Improving time resolution in time-of-flight PET. NuCl Instruments Methods Phys Res Sect A Accel Spectrometers, Detect Assoc Equip. 2011;648:S194-8

72. Moses WW, Janecek M, Spurrier MA, Szupryczynski P, Choong W-S, Melcher CL, et al. Optimization of a LSO-Based Detector Module for Time-of-Flight PET. IEEE Trans Nucl Sci. 2010;57:1570-6.

73. Seifert S, Schaart DR. Improving the Time Resolution of TOF-PET Detectors by Double-Sided Readout. IEEE Trans Nucl Sci. 2015;62:3-11.

74. Jakoby BW, Bercier Y, Watson CC, Bendriem B, Townsend DW. Performance Characteristics of a New LSO PET/CT Scanner With Extended Axial Field-of-View and PSF Reconstruction. IEEE Trans Nucl Sci. 2009;56:633-9.

75. Poon JK, Dahlbom ML, Moses WW, Balakrishnan K, Wang W, Cherry SR, et al. Optimal whole-body PET scanner configurations for different volumes of LSO scintillator: a simulation study. Phys Med Biol. 2012;57:4077-94. 
76. Schmall JP. Karp JS, Werner M, Surti S. Parallax error in long-axial field-of-view PET scanners-a simulation study. Phys Med Biol [lnternet]. IOP Publishing; 2016;61:5443-55. Available from: http:// stacks.iop.org/0031-9155/61/i=14/a=5443?key=crossref.0cbe3af7d86b8b46a740326d7bf165c3

77. Ito M, Hong SJ, Lee JS. Positron emission tomography (PET) detectors with depth-of- interaction (DOI) capability. Biomed Eng Lett. 2011;1:70-81.

78. Rahmim A, Rousset O, Zaidi H. Strategies for Motion Tracking and Correction in PET. PET Clin. 2007:2:251-66.

79. Rahmim A, Tang J, Zaidi H. Four-dimensional (4D) image reconstruction strategies in dynamic PET: Beyond conventional independent frame reconstruction. Med Phys. American Association of Physicists in Medicine; 2009;36:3654.

80. Büther F, Vehren T, Schäfers KP, Schäfers M. Impact of Data-Driven Respiratory Gating in Clinical PET. Radiology. 2016;000:152067.

81. Liu C, Alessio AM, Kinahan PE. Respiratory motion correction for quantitative PET/CT using all detected events with internal-external motion correlation. Med Phys. 2011;38:2715-23.

82. Chan C, Jin X, Fung EK, Naganawa M, Mulnix T, Carson RE, et al. Event-by-event respiratory motion correction for PET with 3D internal-1D external motion correlation. Med Phys. 2013:40:112507.

83. Cheng-Liao J, Qi J. PET image reconstruction with anatomical edge guided level set prior. Phys Med Biol. IOP Publishing; 2011;56:6899-918.

84. Bowsher JE, Johnson VE, Turkington TG, Jaszczak RJ, Floyd CR, Coleman RE. Bayesian reconstruction and use of anatomical a priori information for emission tomography. IEEE Trans Med Imaging 1996;15:673-86.

85. Fessler JA, Hero AO. Penalized maximum-likelihood image reconstruction using space-alternating generalized EM algorithms. IEEE Trans Image Process. 1995;4:1417-29.

86. Hebert T, Leahy R. A generalized EM algorithm for 3-D Bayesian reconstruction from Poisson data using Gibbs priors. IEEE Trans Med Imaging. IEEE; 1989;8:194-202.

87. Lopci E, Chiti A, Castellani MR, Pepe G, Antunovic L, Fanti S, et al. Matched pairs dosimetry: 124l/131 metaiodobenzylguanidine and 124//1311 and 86Y/90Y antibodies. Eur J Nucl Med Mol Imaging. 2011;38 Suppl 1:S28-40.

88. Beattie BJ, Finn RD, Rowland DJ, Pentlow KS. Quantitative imaging of bromine-76 and yttrium-86 with PET: a method for the removal of spurious activity introduced by cascade gamma rays. Med Phys. 2003;30:2410-23.

89. Surti S, Scheuermann R, Karp JS. Correction technique for cascade gammas in 1-124 imaging on a fully-3D, Time-of-Flight PET Scanner. IEEE Trans Nucl Sci. NIH Public Access; 2009;56:653-60.

90. Lubberink M, Herzog H. Quantitative imaging of 1241 and 86 Y with PET. Eur J Nucl Med Mol Imaging 2011;38:10-8

91. Torigian DA, Zaidi H, Kwee TC, Saboury B, Udupa JK, Cho Z-H, et al. PET/MR Imaging: Technical Aspects and Potential Clinical Applications. Radiology. 2013;267:26-44.

92. Keereman V, Mollet P, Berker Y, Schulz V, Vandenberghe S. Challenges and current methods for attenuation correction in PET/MR. Magn Reson Mater Physics, Biol Med. 2013;26:81-98

93. Nuyts J, Dupont P, Stroobants S, Benninck R, Mortelmans L, Suetens P. Simultaneous maximum a posteriori reconstruction of attenuation and activity distributions from emission sinograms. IEEE Trans Med Imaging. IEEE; 1999;18:393-403.

94. Kennedy AS, Salem R. Radioembolization (yttrium-90 microspheres) for primary and metastatic hepatic malignancies. Cancer J. 2010;16:163-75.

95. Tian JH, XU BX, Zhang JM, Dong BW, Liang P, Wang XD. Ultrasound-guided internal radiotherapy using yttrium-90-glass microspheres for liver malignancies. J Nucl Med. 1996;37:958-63.

96. Phillips WT, Bao A, Brenner AJ, Goins BA. Image-guided interventional therapy for cancer with radiotherapeutic nanoparticles. Adv Drug Deliv Rev. 2014;76:39-59.
97. Cistaro A, Quartuccio N, Caobelli F, Piccardo A, Paratore R, Coppolino P, et al. 1241-MIBG: a new promising positron-emitting radiopharmaceutical for the evaluation of neuroblastoma. Nucl Med Rev Cent East Eur. 2015;18:102-6.

98. Beijst C, de Keizer B, Lam MGEH, Janssens GO, Tytgat GAM, de Jong HWAM. A phantom study: Should 124l-mIBG PET/CT replace 1231-mIBG SPECT/CT? Med Phys. John Wiley \& Sons, Ltd; 2017;44:1624-31.

99. Kao Y-H, Steinberg JD, Tay Y-S, Lim GK, Yan J, Townsend DW, et al. Post-radioembolization yttrium-90 PET/CT - part 1: diagnostic reporting. EJNMMI Res. 2013;3:56.

100. Elschot M, Vermolen BJ, Lam MGEH, de Keizer B, van den Bosch MAAJ, de Jong HWAM. Quantitative comparison of PET and Bremsstrahlung SPECT for imaging the in vivo yttrium-90 microsphere distribution after liver radioembolization. PLoS One. 2013;8:e55742.

101. Alavi A, Basu S. Planar and SPECT imaging in the era of PET and PET-CT: can it survive the test of time? Eur J Nucl Med Mol Imaging. 2008;35:1554-9.

102. Beijst C, Kist JW, Elschot M, Viergever MA, Hoekstra OS, De Keizer B, et al. Quantitative comparison of 124I PET/CT and 131I SPECT/CT detectability. J Nucl Med. 2016;57:103-8.

103. Pauwels S, Barone R, Walrand S, Borson-Chazot F, Valkema R, Kvols LK, et al. Practical Dosimetry of Peptide Receptor Radionuclide Therapy with 90Y-Labeled Somatostatin Analogs. J Nucl Med. 2005:46:925-985.

104. Helisch A, Förster GJ, Reber H, Buchholz H-G, Arnold R, Göke B, et al. Pre-therapeutic dosimetry and biodistribution of 86Y-DOTA-Phe1-Tyr3-octreotide versus $111 \mathrm{ln}$-pentetreotide in patients with advanced neuroendocrine tumours. Eur J Nucl Med Mol Imaging. 2004;31:1386冈92.

105. Shyn PB. Interventional positron emission tomography/computed tomography: State-of-the-art. Tech Vasc Interv Radiol. Elsevier; 2013;16:182-90.

106. van der Velden S, Beijst C, Viergever MA, de Jong HWAM. Simultaneous fluoroscopic and nuclear imaging: impact of collimator choice on nuclear image quality. Med Phys. 44:249-61.

107. Jaszczak RJ, Greer KL, Coleman RE. SPECT using a specially designed cone beam collimator. J Nucl Med. 1988;29:1398-405

108. Ducourant T. Wirth T, Bacher G, Bosset B, Vignolle J-M, Blanchon D, et al. Latest advancements in state-of-the-art aSi-based x-ray flat panel detectors. In: Chen G-H, Lo JY, Gilat Schmidt T, editors. Med Imaging 2018 Phys Med Imaging. SPIE; 2018. p. 217

109. Giammarile F, Bodei L, Chiesa C, Flux G, Forrer F, Kraeber-Bodere F, et al. EANM procedure guideline for the treatment of liver cancer and liver metastases with intra-arterial radioactive compounds. Eur J Nucl Med Mol Imaging. 2011;38:1393-406.

110. Gabr A, Kallini JR, Gates VL, Hickey R, Kulik L, Desai K, et al. Same-day 90Y radioembolization: implementing a new treatment paradigm. Eur J Nucl Med Mol Imaging. European Journal of Nuclear Medicine and Molecular Imaging; 2016;43:2353-9.

111. Gates VL, Marshall KG, Salzig K, Williams M, Lewandowski RJ, Salem R. Outpatient Single-Session Yttrium-90 Glass Microsphere Radioembolization. JVasc Interv Radiol. Elsevier; 2014;25:266-70.

112. Garin E, Edeline J, Rolland Y. Letter: High impact of angiographic parameters on MAA and 90Y loaded microsphere uptake correlation. J Nucl Med. 2016;

113. Koppert WJC, van der Velden S, Steenbergen JHL, de Jong HWAM. Impact of intense $x$-ray pulses on a Nal(TI)-based gamma camera. Phys Med Biol. 2018;63:065006.

114. Buettner S, van Vugt JLA, IJzermans J, Groot Koerkamp B. Intrahepatic cholangiocarcinoma: current perspectives. Onco Targets Ther. 2017; Volume 10:1131-42.

115. Endo I, Gonen M, Yopp AC, Dalal KM, Zhou Q, Klimstra D, et al. Intrahepatic Cholangiocarcinoma: Rising Frequency, Improved Survival, and Determinants of Outcome After Resection. Ann Surg. 2008; 248 .

116. Edeline J, Touchefeu Y, Guiu B, Farge O, Tougeron D, Baumgaertner I, et al. Radioembolization Plus Chemotherapy for First-line Treatment of Locally Advanced Intrahepatic Cholangiocarcinoma: A Phase 2 Clinical Trial. JAMA Oncol. 2019:6:51-9. 
117. Swinburne NC, Biederman DM, Besa C, Tabori NE, Fischman AM, Patel RS, et al. Radioembolization for Unresectable Intrahepatic Cholangiocarcinoma: Review of Safety, Response Evaluation Criteria in Solid Tumors 1.1 Imaging Response and Survival. Cancer Biother Radiopharm. 2017;32:161-8.

118. Filippi L, Pelle G, Cianni R, Scopinaro F, Bagni O. Change in total lesion glycolysis and clinical outcome after 90Y radioembolization in intrahepatic cholangiocarcinoma. Nucl Med Biol. Elsevier Inc 2015;42:59-64.

119. Mouli S, Memon K, Baker T, Benson AB, Mulcahy MF, Gupta R, et al. Yttrium-90 Radioembolization for Intrahepatic Cholangiocarcinoma: Safety, Response, and Survival Analysis. J Vasc Interv Radiol. 2013;24:1227-34.

120. Gangi A, Shah J, Hatfield N, Smith J, Sweeney J, Choi J, et al. Intrahepatic Cholangiocarcinoma Treated with Transarterial Yttrium-90 Glass Microsphere Radioembolization: Results of a Single Institution Retrospective Study. J Vasc Interv Radiol. United States; 2018;29:1101-8.

121. Shaker TM, Chung C, Varma MK, Doherty MG, Wolf AM, Chung MH, et al. Is there a role for Ytrrium-90 in the treatment of unresectable and metastatic intrahepatic cholangiocarcinoma? Am J Surg Elsevier; 2018;215:467-70.

122. Bastiaannet R, Kappadath SC, Kunnen B, Braat AJAT, Lam MGEH, de Jong HWAM. The physics of radioembolization. EJNMMI Phys. EJNMMI Physics; 2018;5:22.

123. Chiesa C, Mira M, Maccauro M, Spreafico C, Romito R, Morosi C, et al. Radioembolization of hepatocarcinoma with $90 \mathrm{Y}$ glass microspheres: development of an individualized treatment planning strategy based on dosimetry and radiobiology. Eur J Nucl Med Mol Imaging. 2015;42:171838.

124. van den Hoven AF, Rosenbaum CENM, Elias SG, de Jong HWAM, Koopman M, Verkooijen HM, et al. Insights into the Dose-Response Relationship of Radioembolization with Resin 90Y-Microspheres: A Prospective Cohort Study in Patients with Colorectal Cancer Liver Metastases. J Nucl Med 2016;57:1014-9.

125. Kappadath SC, Mikell J, Balagopal A, Baladandayuthapani V, Kaseb A, Mahvash A. Hepatocellular Carcinoma Tumor Dose Response After 90 Y-radioembolization With Glass Voxel Dosimetry. Radiat Oncol Biol. Elsevier Inc; 2018;102:451-61.

126. Garin E, Lenoir L, Rolland Y, Edeline J, Mesbah H, Laffont S, et al. Dosimetry Based on 99mTcMacroaggregated Albumin SPECT/CT Accurately Predicts Tumor Response and Survival in Hepatocellular Carcinoma Patients Treated with 90Y-Loaded Glass Microspheres: Preliminary Results. J Nucl Med. 2012;53:255-63,

127. Sirtex Medical. SIR-Spheres Yttrium-90 Microspheres package insert (CR2014) [Internet]. 2016. Available from: https://www.sirtex.com/media/155127/pi-ec-12.pdf

128. Wahl RL, Jacene H, Kasamon Y, Lodge MA. From RECIST to PERCIST: Evolving Considerations for PET Response Criteria in Solid Tumors. J Nucl Med. 2009;50:122-50.

129. Klein S, Staring M, Murphy K, Viergever MA, Pluim JP. elastix: A Toolbox for Intensity-Based Medical Image Registration. IEEE Trans Med Imaging. 2010;29:196-205.

130. Fedorov A, Beichel R, Kalpathy-Cramer J, Finet J, Fillion-Robin J-C, Pujol S, et al. 3D Slicer as an Image Computing Platform for the Quantitative Imaging Network. Magn Reson Imaging. 2012;30:1323-41.

131. Eisenhauer EA, Therasse P, Bogaerts J, Schwartz LH, Sargent D, Ford R, et al. New response evaluation criteria in solid tumours: revised RECIST quideline (version 1.1). Eur J Cancer. England: 2009:45:228-47.

132. O JH, Lodge MA, Wahl RL. Practical PERCIST: A Simplified Guide to PET Response Criteria in Solid Tumors 1.0. Radiology. 2016;280:576-84.

133. Braat MNGJA, van Erpecum KJ, Zonnenberg BA, van den Bosch MAJ, Lam MGEH. Radioembolizationinduced liver disease: a systematic review. Eur J Gastroenterol Hepatol. England; 2017;29:144-52.

134. Pasciak AS, Abiola G, Liddell RP, Crookston N, Besharati S, Donahue D, et al. The number of microspheres in $Y 90$ radioembolization directly affects normal tissue radiation exposure. Eur J Nucl Med Mol Imaging. Germany; 2020;47:816-27.
135. Strigari L, Sciuto R, Rea S, Carpanese L, Pizzi G, Soriani A, et al. Efficacy and toxicity related to treatment of hepatocellular carcinoma with 90Y-SIR spheres: radiobiologic considerations. J Nucl Med. United States; 2010:51:1377-85.

136. Nezami N, Kokabi N, Camacho JC, Schuster DM, Xing M, Kim HS. Y90 radioembolization dosimetry using a simple semi-quantitative method in intrahepatic cholangiocarcinoma: Glass versus resin microspheres. Nucl Med Biol. Elsevier Inc.; 2018;59:22-8.

137. Buettner S, Braat AJAT, Margonis GA, Brown DB, Taylor KB, Borgmann AJ, et al. Yttrium-90 Radioembolization in Intrahepatic Cholangiocarcinoma: A Multicenter Retrospective Analysis. JVasc Interv Radiol. 2020;31:1035-1043.e2

138. Manceau V, Palard X, Rolland Y, Pracht M, Le Sourd S, Laffont $S$, et al. A MAA-based dosimetric study in patients with intrahepatic cholangiocarcinoma treated with a combination of chemotherapy and

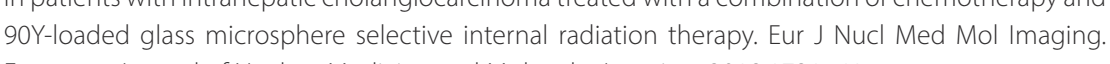
European Journal of Nuclear Medicine and Molecular Imaging; 2018;1731-41.

139. Kunnen B, Beijst C, Lam MGEH, Viergever MA, de Jong HWAM. Comparison of the Biograph Vision and Biograph $\mathrm{mCT}$ for quantitative 90Y PET/CT imaging for radioembolisation. EJNMMI Phys. 2020;7:14.

140. Kunnen B, van der Velden S, Bastiaannet $R$, Lam MGEH, Viergever MA, de Jong HWAM Radioembolization lung shunt estimation based on a $90 \mathrm{Y}$ pretreatment procedure: A phantom study. Med Phys. 2018:45:4744-53.

141. Kunnen B, Dietze MMA, Braat AJAT, Lam MGEH, Viergever MA, Jong HWAM. Feasibility of imaging 90 Y microspheres at diagnostic activity levels for hepatic radioembolization treatment planning. Med Phys. John Wiley \& Sons, Ltd; 2020;47:1105-14.

142. Ebbers SC, Kunnen B, van Nierop BJ, Bemelmans JLM, Krijger GC, Lam MGEH, et al. Verification Study of Residual Activity Measurements After Yttrium-90 Radioembolization with Glass Microspheres. Cardiovasc Intervent Radio. 2020:

143. Khazaee M, Kamali-Asl A, Geramifar P, Rahmim A. Low-dose 90Y PET/CT imaging optimized for lesion detectability and quantitative accuracy. Nucl Med Commun. 2017;1.

144. Braat AJAT, Prince JF, van Rooii R, Bruijnen RCG, van den Bosch MAAJ, Lam MGEH. Safety analysis of holmium-166 microsphere scout dose imaging during radioembolisation work-up: A cohort study. Eur Radiol. European Radiology; 2018;28:920-8.

145. Prince JF, van Rooij R, Bol GH, de Jong HW, van den Bosch MA, Lam MG. Safety of a Scout Dose Preceding Hepatic Radioembolization with 166Ho Microspheres. J Nucl Med. 2015;56:817-23.

146. Prince JF, van den Bosch MAAJ, Nijsen JFW, Smits ML, van den Hoven AF, Nikolakopoulos S, et at. Efficacy of radioembolization with holmium-166 microspheres in salvage patients with liver metastases: a phase 2 study. J Nucl Med [Internet]. 2017; Available from: http://jinm.snmjournals. org/content/early/2017/09/15/jnumed.117.197194.abstract

147. Selwyn RG, Nickles RJ, Thomadsen BR, DeWerd LA, Micka JA. A new internal pair production branching ratio of 90Y: The development of a non-destructive assay for 90Y and 90Sr. Appl Radiat Isot. Pergamon; 2007;65:318-27.

148. Willowson K, Forwood N, Jakoby BW, Smith AM, Bailey DL. Quantitative (90)Y image reconstruction in PET. Med Phys. 2012;39:7153-9.

149. Carlier T, Willowson KP, Fourkal E, Bailey DL, Doss M, Conti M. (90)Y -PET imaging: Exploring limitations and accuracy under conditions of low counts and high random fraction. Med Phys. 2015;42:4295.

150. Attarwala AA, Molina-Duran F, Büsing K-A, Schönberg SO, Bailey DL, Willowson K, et al. Quantitative and qualitative assessment of Yttrium-90 PET/CT imaging. PLOS One. 2014;9:e1 10401.

151. Carlier T, Eugène T, Bodet-Milin C, Garin E, Ansquer C, Rousseau C, et al. Assessment of acquisition protocols for routine imaging of Y-90 using PET/CT. EJNMMI Res. 2013;3:11.

152. Pasciak AS, Bourgeois AC, McKinney JM, Chang TT, Osborne DR, Acuff SN, et al. Radioembolization and the Dynamic Role of $90 \mathrm{YPET} / \mathrm{CT}$. Front Oncol. 2014;4.1-12. 
153. Park M-A, Mahmood A, Zimmerman RE, Limpa-Amara N, Makrigiorgos GM, Moore SC. Adsorption of metallic radionuclides on plastic phantom walls. Med Phys. 2008;35:1606-10.

154. Floridi C, Pesapane F, Angileri SA, De Palma D, Fontana F, Caspani F, et al. Yttrium-90 radioembolization treatment for unresectable hepatocellular carcinoma: a single-centre prognostic factors analysis. Med Oncol. Springer US; 2017;34:1-12.

155. Kao YH, Steinberg JD, Tay YS, Lim GKY, Yan J, Townsend DW, et al. Post-radioembolization yttrium-90 PET/CT-part 2: Dose-response and tumor predictive dosimetry for resin microspheres. EJNMMI Res. EJNMMI Research; 2013:3:1-27.

156. Van Slambrouck K, Stute S, Comtat C, Sibomana M, Van Velden FHP, Boellaard R, et al. Bias reduction for low-statistics PET: Maximum likelihood reconstruction with a modified poisson distribution. IEEE Trans Med Imaging. 2015;34:126-36.

157. Willowson KP, Tapner M, Team Q U E S T Investigator, Bailey DL. A multicentre comparison of quantitative (90)Y PET/CT for dosimetric purposes after radioembolization with resin microspheres: The QUEST Phantom Study. Eur J Nucl Med Mol Imaging. 2015;42:1202-22.

158. Gabr A, Kallini JR, Gates VL, Hickey R, Kulik L, Desai K, et al. Same-day 90Y radioembolization: implementing a new treatment paradigm. Eur J Nucl Med Mol Imaging [Internet]. European Journat of Nuclear Medicine and Molecular Imaging: 2016:43.2353-9. Available from: http $/ / d x$ dol org/10.1007/s00259-016-3438-x

159. Ho S, Lau WY, Leung TWT, Chan M, Ngar YK, Johnson PJ, et al. Partition model for estimating radiation doses from yttrium-90 microspheres in treating hepatic tumours. Eur J Nucl Med. 1996;23:947-52.

160. Goldin D, Campbell J, Emerson S, Bahoura L, George D, Reich S, et al. Tumor-to-normal particle deposition ratio in primary versus secondary liver malignancies: impact on tumor dose and radioembolization treatment planning. J Vasc Interv Radiol. Elsevier; 2014;25:589-90.

161. Rong X, Du Y, Ljungberg M, Rault E, Vandenberghe S, Frey EC. Development and evaluation of an improved quantitative 90Y bremsstrahlung SPECT method. Med Phys. John Wiley \& Sons, Ltd; 2012:39:2346-58.

162. Alsultan AA, Smits ML, Barentsz MW, Braat AJAT, Lam MGEH. The value of yttrium-90 PET/CT after hepatic radioembolization: a pictorial essay. Clin Transl Imaging. 2019;7:303-12.

163. Dewaraja YK, Devasia T, Kaza RK, Mikell JK, Owen D, Roberson PL, et al. Prediction of Tumor Control in 90 Y Radioembolization by Logit Models with PET/CT-Based Dose Metrics. J Nucl Med. 2020;61:10411.

164. Mazzaferro V, Sposito C, Bhoori S, Romito R, Chiesa C, Morosi C, et al. Yttrium-90 radioembolization for intermediate-advanced hepatocellular carcinoma: A phase 2 study. Hepatology. John Wiley \& Sons, Ltd; 2013:57:1826-37.

165. Yue J, Mauxion T, Reyes DK, Lodge MA, Hobbs RF, Rong X, et al. Comparison of quantitative Y-90 SPECT and non-time-of-flight PET imaging in post-therapy radioembolization of liver cancer. Med Phys. 2016;43:5779-90.

166. Lea WB, Tapp KN, Tann M, Hutchins GD, Fletcher JW, Johnson MS. Microsphere localization and dose quantification using positron emission tomography/CT following hepatic intraarterial radioembolization with yttrium-90 in patients with advanced hepatocellular carcinoma. J Vasc Interv Radiol. Elsevier; 2014;25:1595-603.

167. Ithan H, Goritschan A, Paprottka P. Jakobs TF, Fendler WP. Todica A, et al. Predictive Value of 99mTcMAA SPECT for 90Y-Labeled Resin Microsphere Distribution in Radioembolization of Primary and Secondary Hepatic Tumors. J Nucl Med. 2015;56:1654-60.

168. Sluis J Van, Jong J De, Schaar J, Noordzij W, Snick van P, Dierckx R, et al. Performance characteristics of the digital Biograph Vision PET / CT system. J Nucl Med. 2019;

169. van Sluis J, Boellaard R, Somasundaram A, van Snick PH, Borra RJH, Dierckx RAJO, et al. Image Quality and Semiquantitative Measurements on the Biograph Vision PET/CT System: Initial Experiences and Comparison with the Biograph mCT. J Nucl Med. 2020;61:129-35.
170. Karlberg AM, Sæther O, Eikenes L, Goa PE. Quantitative comparison of PET performance-Siemens Biograph mCT and mMR. EJNMMI Phys. 2016/02/25. Springer International Publishing; 2016;3:5.

171. Conti M, Eriksson L, Rothfuss H, Sjoeholm T, Townsend D, Rosenqvist G, et al. Characterization of 176Lu background in LSO-based PET scanners. Phys Med Biol. IOP Publishing; 2017;62:3700-11.

172. Siman W, Kappadath SC. Comparison of Step-and-Shoot and Continuous-Bed-Motion PET Modes of Acquisition for Limited-View Organ Scans. J Nucl Med Technol. 2017;45:290-6.

173. van der Velden S, Dietze MMA, Viergever MA, de Jong HWAM. Fast technetium-99m liver SPECT for evaluation of the pretreatment procedure for radioembolization dosimetry. Med Phys. 2018;

174. Dietze MMA, Bastiaannet R, Kunnen B, van der Velden S, Lam MGEH, Viergever MA, et al. Respiratory Motion Compensation in Interventional Liver SPECT using Simultaneous Fluoroscopic and Nuclear Imaging. Med Phys. John Wiley \& Sons, Ltd; 2019;46:3496-507.

175. Koppert WJC, Dietze MMA, van der Velden S, Steenbergen JHL, de Jong HWAM. A comparative study of $\mathrm{Nal}(\mathrm{TI}), \mathrm{CeBr}$, and CZT for use in a real-time simultaneous nuclear and fluoroscopic dual-layer detector. Phys Med Biol. IOP Publishing; 2019;64:135012.

176. Berrocal J, Saperstein L, Grube B, Horowitz NR, Chagpar AB, Killelea BK, et al. Intraoperative Injection of Technetium-99m Sulfur Colloid for Sentinel Lymph Node Biopsy in Breast Cancer Patients: A Single Institution Experience. Surg Res Pract. 2017/04/13. Hindawi; 2017;2017:5924802.

177. Bluemel C, Schnelzer A, Okur A, Ehlerding A, Paepke S, Scheidhauer K, et al. Freehand SPECT for image-guided sentinel lymph node biopsy in breast cancer. Eur J Nucl Med Mol Imaging. 2013;40:1656-61.

178. Heuveling DA, van Weert S, Karagozoglu KH, de Bree R. Evaluation of the use of freehand SPECT for sentinel node biopsy in early stage oral carcinoma. Oral Oncol. 2015;51:287-90.

179. de Bree R, Pouw B, Heuveling DA, Castelijns JA. Fusion of Freehand SPECT and Ultrasound to Perform Ultrasound-Guided Fine-Needle Aspiration Cytology of Sentinel Nodes in Head and Neck Cancer. AJNR Am J Neuroradiol. 2015:36:2153-8. 


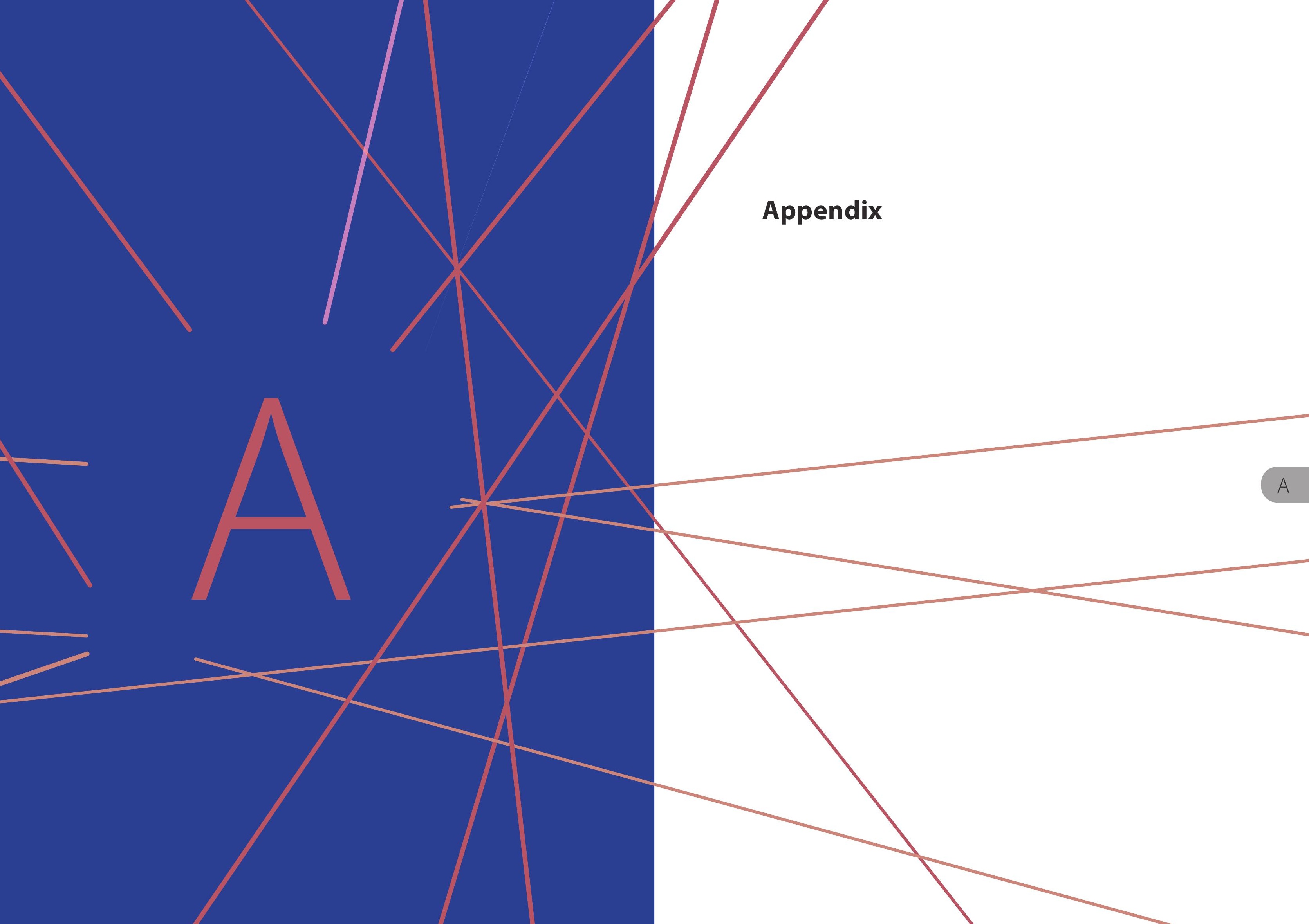




\section{List of publications}

B Kunnen, R. van Rooij, M. Stella, M.G.E.H. Lam, H.W.A.M. de Jong, A.J.A.T. Braat "Comparison of three different therapeutic particles in radioembolization of locally advanced intrahepatic cholangiocarcinoma". Submitted

M.M.A. Dietze, B. Kunnen, M.G.E.H. Lam, H.W.A.M. de Jong. "Interventional respiratory motion compensation by simultaneous fluoroscopic and nuclear imaging". Submitted

M.M.A. Dietze, B. Kunnen, F. Brontsema, P. Ramaekers, C. Beijst, M. Afifah, A.J.A.T. Braat M.G.E.H. Lam, H.W.A.M. de Jong. "A compact and mobile hybrid c-arm scanner for simultaneous and fluoroscopic image guidance". Submitted

M.M.A. Dietze, B. Kunnen, M. Stella, H.W.A.M. de Jong. "Monte Carlo-based scatter correction for the SMARTZOOM collimator". EJNMMI Physics, 7 (1): 49; (2020)

S.C. Ebbers, B. Kunnen, B.J. van Nierop, J.L.M. Bemelmans, G.C. Krijger, M.G.E.H. Lam, A.J.A.T. Braat. "Verification study of residual activity measurements after yttrium-90 radioembolization glass microspheres". Cardio Vascular and Interventional Radiology, doi:10.1007/s00270-020-02504-7; (2020)

B. Kunnen, C. Beijst, M.G.E.H. Lam, M.A. Viergever, H.W.A.M. de Jong. "Comparison of the Biograph Vision and Biograph $\mathrm{mCT}$ for quantitative ${ }^{90} \mathrm{Y}$ PET/CT imaging for radioembolisation". EJNMMI Physics, 7 (1): 14; (2020)

M.M.A. Dietze, B. Kunnen, C. Beijst, H.W.A.M. de Jong. "Adaptive scan duration in SPECT: evaluation for radioembolization". Medical Physics, 47(5):2128-2138; (2020)

B. Kunnen, M.M.A. Dietze, A.J.A.T. Braat, M.G.E.H. Lam, M.A. Viergever, H.W.A.M. de Jong. "Feasibility of imaging ${ }^{90} \mathrm{Y}$ microspheres at diagnostic activity levels for hepatic radioembolization treatment planning". Medical Physics, 47 (3): 1105-1114; (2020)

C.M. Macdonald, B. Kunnen, C. Stayner, M.R. Eccles. "Designing phantoms to accurately replicate circular depolarization in biological scattering media". Applied Optics, 58 (35): 9577-9584; (2019)

M.M.A. Dietze, W. Branderhorst, B. Kunnen, M.A. Viergever, H.W.A.M. de Jong. "Accelerated SPECT image reconstruction with FBP and an image enhancement convolutional neural network". EJNMMI Physics, 6 (1): 14; (2019)
M.M.A. Dietze, R. Bastiaannet, B. Kunnen, S. van der Velden, M.G.E.H. Lam, M.A. Viergever, H.W.A.M. de Jong. "Respiratory motion compensation in interventional liver SPECT using simultaneous fluoroscopic and nuclear imaging". Medical Physics, 46 (8): 3496-3507; (2019)

M.M.A. Dietze, B. Kunnen, S. van der Velden, J.H.L. Steenbergen, W.J.L. Koppert, M.A. Viergever, H.W.A.M. de Jong. "Performance of a dual-layer scanner for hybrid SPECT/CBCT". Physics in Medicine\&Biology, 64 (10): 105020 (2019)

S. van der Velden*, B. Kunnen*, W.J.L. Koppert, J.H.L. Steenbergen, M.M.A. Dietze, C. Beijst, M.A. Viergever, M.G.E.H. Lam, H.W.A.M. de Jong. "A dual-layer detector for simultaneous fluoroscopic and nuclear imaging". Radiology, 290 (3): 833-838; (2019) *contributed equally to this work

B. Kunnen, S. van der Velden, R. Bastiaannet, M.G.E.H. Lam, M.A. Viergever, H.W.A.M. de Jong. "Radioembolization lung shunt estimation based on a ${ }^{90} Y$ pretreatment procedure: a phantom study". Medical Physics, 45 (10): 4744-4753; (2018)

R. Bastiaannet, S. Kappadath, B. Kunnen, A.J.A.T. Braat, M.G.E.H. Lam, H.W.A.M. de Jong. "The physics of radioembolization". EJNMMI Physics, 5 (1): 22; (2018)

R. Bastiaannet, C. van Roekel, B. Kunnen, M.G.E.H. Lam, H.W.A.M. de Jong. "Is diffusionweighted MRI really superior to PET/CT in predicting survival after radioembolization?". Radiology, 289 (1): 274-275; (2018)

C. Beijst, B. Kunnen, M.G.E.H. Lam, H.W.A.M. de Jong. "Technical advances in image guidance of radionuclide therapy". Journal of Nuclear Medicine Technology, 45 (4): 272279; (2017)

B. Kunnen, C.M. Macdonald, A. Doronin, S.L. Jacques, M.R. Eccles, I.V. Meglinski. “Application of circularly polarized light for non-invasive diagnosis of cancerous tissues and turbid-like scattering media". Journal of Biophotonics, 8 (4): 317-323; (2015) 


\section{Appendix}

Britt Kunnen was born on the $16^{\text {th }}$ of September 1990 in Amsterdam, the Netherlands. After spending her childhood in Diemen, she started high school at the Montessori Lyceum Amsterdam, where she graduated in 2008. That same year she started her bachelor Medische Natuurwetenschappen at the VU University Amsterdam. During her studies she was an active member of the study association Mens, where she joined the board as the secretary in 2011

She continued her studies at the VU University Amsterdam with a master Medical Physics. During those years, she performed a seven month internship at the Department of Radiotherapy of the Netherlands Cancer Institute. The final six months of her masters were spend at the University of Otago (Dunedin, New Zealand) where she performed a research project in the field of biophotonics.

After obtaining her master degree (cum laude) in 2014, she returned to Dunedin to continue the work of her masters project as a research assistant. In 2016 she decided she wanted to return to a more clinical work field, and moved to Utrecht, the Netherlands, to start her PhD at the Department of Radiology and Nuclear Medicine of the University Medical Center Utrecht, under the supervision of Hugo de Jong. The results of her PhD are described in this thesis.

Britt will continue to work in the field of medical physics as a Medical Physicist resident at the Department of Radiotherapy of the Erasmus UMC, Rotterdam, the Netherlands. 


\section{Dankwoord}

Het allerlaatste hoofdstuk wat ik schrijf; het dankwoord. Na een dikke vier jaar is het tijd om iedereen te bedanken die dit proefschrift mede mogelijk heeft gemaakt. Bij het UMC Utrecht ben ik stilletjes vertrokken in een periode waarin de meesten thuis aan het werk waren. Een afscheid zat er helaas niet in. En ook nu, aan het begin van de tweede golf, gaat er een streep door mijn promotiefeestje. En dat betekent ook een streep door de biertjes en bitterballen waar ik iedereen graag op had getrakteerd, want die hebben jullie na deze vier jaar wel verdiend. Het feestje houden jullie van me tegoed. Voor nu zullen jullie het als bedankje met dit hoofdstuk moeten doen. Ik ga niet iedereen bij naam noemen, maar het feit dat je dit proefschrift in je handen hebt en bij het laatste hoofdstuk bent gekomen (ook als je achteraan bent begonnen), betekent dat je op een manier heb bijgedragen aan mijn promotietraject. Bedankt daarvoor!

Allereerst wil ik mijn promotoren bedanken. Hugo, je hebt een ontzettend leuke groep fysici om je heen verzameld. De sfeer in de groep is uitstekend en alles kan altijd gezegd worden. Jouw openheid maakt dit mogelijk, en heb ik erg gewaardeerd. Ik ben blij dat ik onderdeel heb mogen uitmaken van de IXSI groep, en dat je me daarnaast de vrijheid hebt gegeven om andere projecten op te pakken. Max, ondanks de vele PhD studenten die je hebt begeleid, was je altijd een van de eersten die mijn manuscripten had gelezen. Dank dat ik onderdeel van het ISI mocht zijn. Marnix, jouw optimisme en enthousiasme werken motiverend. Dank voor je kritische klinische blik, die me dwongen goed na te denken over de klinische toepasbaarheid van mijn werk.

Sandra, Remco, Martijn, Fasco en Daan, met jullie had ik me geen betere kamergenoten kunnen wensen. Er was op het kantoor ruimte voor alles: van slap gelul tot verhitte discussies, van vragen over statistiek of analyses tot ergernissen over reviewcommentaar, en van het bespreken van weekendplannen tot het filosoferen over levensvragen. Ik heb me vanaf mijn eerste werkdag op mijn plek gevoeld, en daar hebben jullie een grote rol in gespeeld. Als mede PhDers begrepen jullie als geen ander welke hobbels je tegenkomt tijdens je promotie, en ons kantoor is voor mij altijd de plek geweest waar ik deze hobbels zonder enige terughoudendheid kon bespreken.

Wilco, Leo, Pascal, Frank en Maryam, de IXSI is van een bij elkaar geraapt zooitje onderdelen veranderd in een echte scanner. En dit hebben we te danken aan jullie doorzettingsvermogen. Jullie hebben oneindig veel geklust aan de IXSI en ervoor gezorgd dat ik zonder problemen mijn experimenten kon doen. Daarnaast zorgden jullie ook nog eens voor de vrolijke noot op de verdieping. Ook als dingen weer eens langer duurden dan je zou willen, of er geneuzel was over documentatie, bleven jullie altijd lachen. Dank voor de vele gezellige koffiemomentjes en dat jullie mij er steeds aan hebben helpen herinneren dat niet alles om het aantal gepubliceerde papers draait.

Martina, Achmed en Sander, wat een geluk om twee artsen en een Quirem expert op de verdieping te hebben gekregen! De laagdrempeligheid waarmee ik bij jullie met praktische vragen aan kon kloppen heeft mijn leven als promovendus werkelijk makkelijker gemaakt. En daarnaast weten jullie van promoveren echt een feestje te maken. Jullie kantoor voelde als een soort van huiskamer, en waar ik gewend was om een publicatie te vieren met taart, toveren jullie gewoon bier, bubbels, en bittergarnituur tevoorschijn. Een uitstekend idee

Woutjan, eens beneden begonnen, maar ondertussen houdt je al best lang stand boven. Het was fijn om met jou iemand te hebben die zowel weet hoe het leven in NieuwZeeland eraan toe gaat, als iemand die tips kan geven over het verbouwen van je huis.

Rob, Arnold, Bastiaan, Casper en Bas, de fysici van beneden. Bij jullie stond de deur altijd open en kon ik met al mijn vragen terecht. Samen lunchen, christmasparty, fietsenrally, congres of een weekendje Ardennen, met jullie is het altijd gezellig!

Dan zijn er ook zeker nog mensen van buiten de Klinische Fysica groep die ik wil bedanken. Remmert, de helpende hand in het lab. Je hielp niet alleen mee met het uitdenken van een experiment, je zorgde ook dat alle benodigdheden op het lab aanwezig waren, en was nooit te beroerd om bij te springen als jouw expertise nodig was. Arthur, zelfs met jouw drukke werkagenda kon je altijd tijd vrijmaken. Samenwerken met jou zorgt ervoor dat de vaart er lekker in zit en dat er flinke stappen gezet worden. Margot en Caren, jullie kunnen de lekkerste taarten bakken! Voor al jullie hulp ben ik jullie minstens zoveel taart verschuldigd. Laboranten van de nucleaire geneeskunde, dank voor het klaarmaken van alle activiteit en het helpen scannen. Als ik een avond moest meten was niets zo fijn om de scanruimte binnen te lopen en te zien dat jullie de collimatoren alvast voor me verwisseld hadden. ledereen van het ISI: dank voor het Ardennenweekend, Sinterklaasavond, en alle gezellige etentjes, borrels en feestjes!

Wie ik natuurlijk absoluut niet kan vergeten zijn Ben, Annemieke en Storm. Jullie hebben altijd ongekend vertrouwen gehad dat ik mijn promotie succesvol zou afronden. Al vanaf dag 1 ging het erover wie mijn paranimfen mochten zijn. Het is dan ook jammer dat ik alleen 2 paranimfen mag kiezen, en dat ik de rol van pedel niet aan Ben kan geven.

En dan, last but not least, Rens: jij bent de beste! 